\title{
Molecular Conformation of Bent-Core Molecules Affected by Chiral Side Chains Dictates Polymorphism and Chirality in Organic Nano- and Microfilaments
}

Jiao Liu, Sasan Shadpour, Marianne E. Prévôt, Michael Chirgwin, Ahlam Nemati, Elda Hegmann, Robert P. Lemieux, and Torsten Hegmann*

\section{SUPPORTING INFORMATION (SI)}

Section

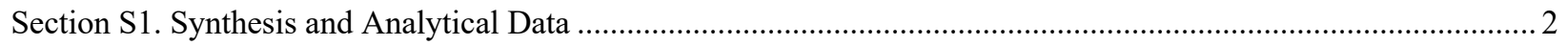

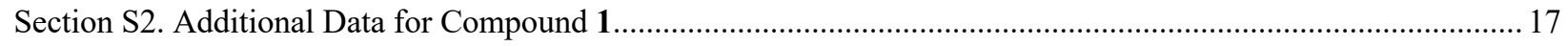

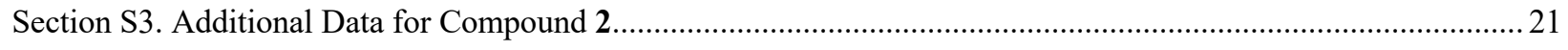

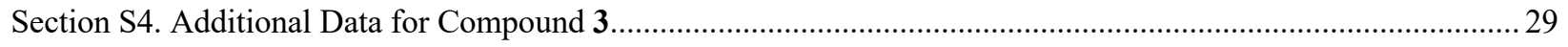

Section S5. Additional Data for Compound 4 ………....................................................................................... 34

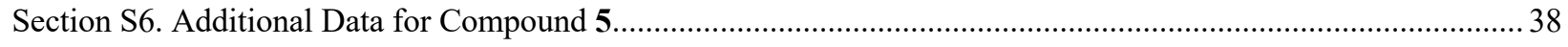

Section S7. Additional Data for Compounds 3 - 5 in $n$-Hexane Dispersions ....................................................... 43

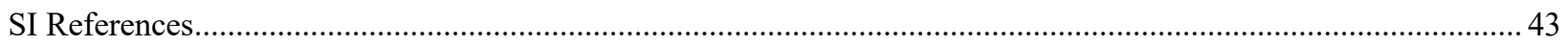




\section{Section S1. Synthesis and Analytical Data}

\section{Materials}

Commercially available starting materials and reagents were used as received without further purification unless otherwise noted. All organic solvents used for synthesis were EMD Millipore-grade and purified by a PureSolv solvent purification system (Innovative Technology Inc.). Dichloromethane $\left(\mathrm{CH}_{2} \mathrm{Cl}_{2}\right)$ and benzene $\left(\mathrm{C}_{6} \mathrm{H}_{6}\right)$ for syntheses were dried by $4-\AA$ molecular sieves for $24 \mathrm{~h}$. Tetrahydrofuran (THF) was dried by sodium-benzophenone and distilled under a nitrogen atmosphere. All glassware used for the reactions were cleaned and dried overnight at $140{ }^{\circ} \mathrm{C}$ in an oven. All reagents were purchased from Sigma Aldrich (and used as received) except for 4-n-octyloxybiphenyl-4'-carboxylic acid and 4- $n$-decyloxybiphenyl-4'-carboxylic acid (purchased from Synthon Chemicals GmbH \& Co. KG) and 1-ethyl-3-(3-dimethylaminopropyl) carbodiimide (EDCI) (purchased from TCI).

\section{Methods}

${ }^{1} \mathrm{H}(400 \mathrm{MHz})$ and ${ }^{13} \mathrm{C}$ NMR $(100 \mathrm{MHz})$ spectra were obtained using a Bruker Avance $400 \mathrm{MHz}$ spectrometer in $\mathrm{CDCl}_{3} .{ }^{1} \mathrm{H}$ NMR spectra are reported in parts per million $(\delta)$ relative to residual solvent peaks (7.26 ppm for $\mathrm{CDCl}_{3}$ or $0.08 \mathrm{ppm}$ for tetramethylsilane, TMS). ${ }^{13} \mathrm{C}$ NMR spectra are reported in parts per million $(\delta)$ relative to residual solvent peaks $\left(77.00\right.$ for $\mathrm{CDCl}_{3}$ ). Elemental analysis was done using a TrueSpec Micro CHNS analyzer (Leco Corp., St. Joseph, MI). 


\section{Synthesis Route}

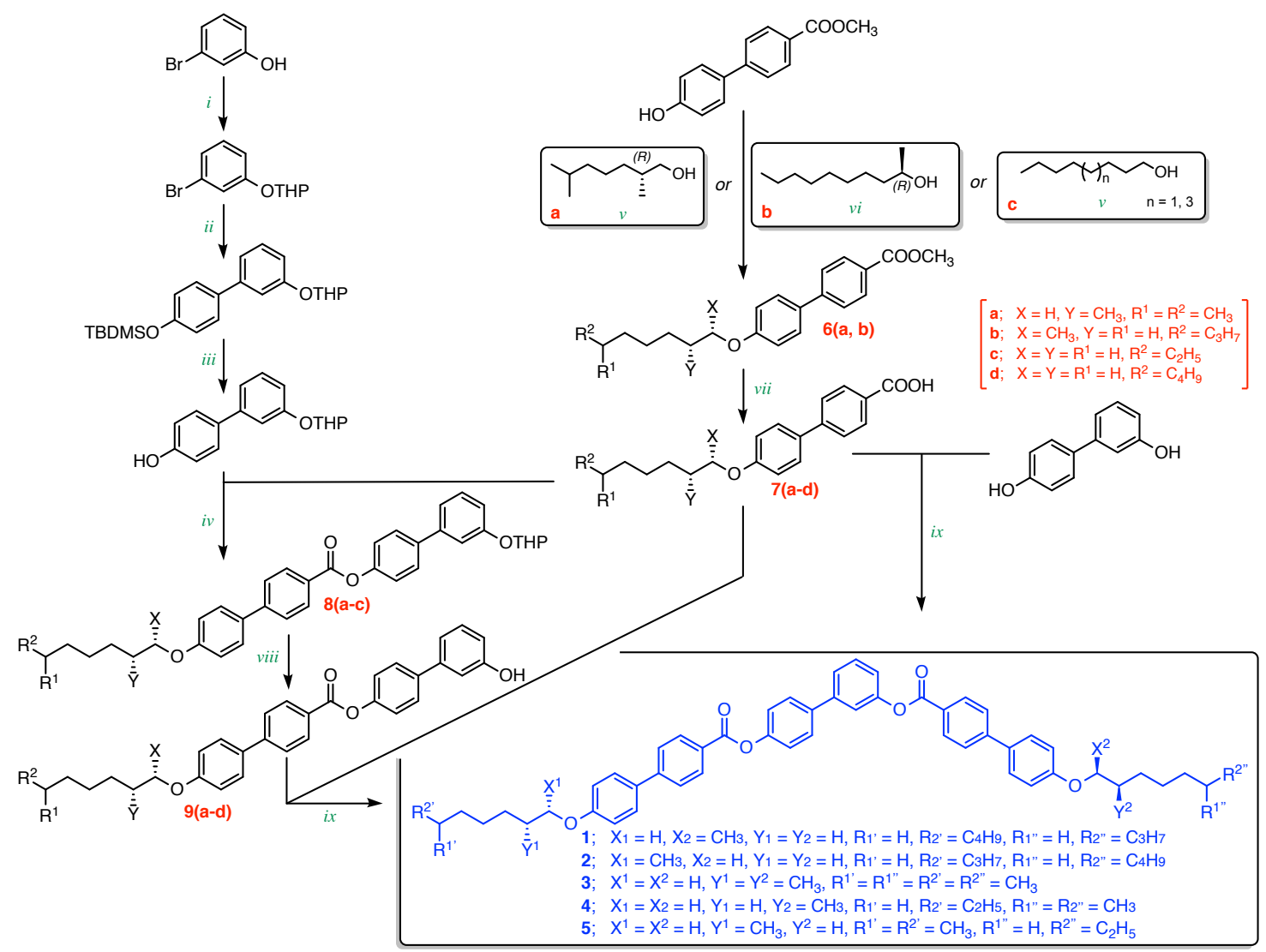

Scheme S1. Synthetic route pursed to obtain compounds $\mathbf{1}-\mathbf{5}$. The synthesis process of the various intermediates has been reported previously. ${ }^{1-4}$ (i) DHP, $p$-TSA, (ii) TBDMSO-Ph-B $(\mathrm{OH})_{2}, \mathrm{Pd}\left(\mathrm{PPh}_{3}\right)_{4}$, $\mathrm{C}_{6} \mathrm{H}_{6}, 2 \mathrm{M}$ aq. $\mathrm{Na}_{2} \mathrm{CO}_{3}$, (iii) TBAF, THF, (iv) conc. $\mathrm{HCl}$, (v, vi) DIAD, $\mathrm{PPh}_{3}$, (vii) conc. $\mathrm{HCl}$, (viii) conc. $\mathrm{HCl}$, and (ix) EDCI, DMAP.

Intermediates (not previously published) - (published ones can be found in references cited ${ }^{1-4}$ )

\section{Methyl 4-(4-\{[(2R)-2,6-dimethylheptyl]oxy\}phenyl)benzoate (6a)}

In a $\mathrm{N}_{2}$-purged two-necked round flask, methyl 4'-hydroxy(1,1'-biphenyl)-4-carboxylate (1.069 $\mathrm{g}, 4.68 \mathrm{mmol})$, triphenylphosphine $(1.613 \mathrm{~g}, 6.10 \mathrm{mmol})$, and $(R)$-3,7-dimethyl-1-octanol (878.2 $\mathrm{mg}, 6.10 \mathrm{mmol}$ ) were dissolved in $10 \mathrm{~mL}$ of tetrahydrofuran (THF). After stirring for 10 minutes, $2 \mathrm{~mL}$ THF with diisopropyl azodicarboxylate (DIAD) $(1.223 \mathrm{~mL}, 6.10 \mathrm{mmol})$ was dropwise added to this mixture. The reaction was magnetically stirred in an ice bath for 5 hours; the reaction was monitored by thin layer chromatography (TLC). After completion of the reaction, the solution was washed with saturated $\mathrm{NaCl}$ solution three times and extracted by $\mathrm{CHCl}_{3}$, then dried with anhydrous $\mathrm{Na}_{2} \mathrm{SO}_{4}$. The filtrate was concentrated by rotary evaporation; the residue 
was purified by column chromatography with $\mathrm{CHCl}_{3}$ to yield a white solid $(1.3 \mathrm{~g}, 80 \%)$. ${ }^{1} \mathrm{H}$ NMR (CDCl $3,400 \mathrm{MHz}, \delta / \mathrm{ppm}): \delta 8.10(\mathrm{dd}, 2 \mathrm{H}, J=8.52 \mathrm{~Hz}, 4.88 \mathrm{~Hz}$, biphenyl), 7.64 (dd, 2H, $J=8.80 \mathrm{~Hz}, 2.10 \mathrm{~Hz}$, biphenyl), 7.59 (dd, 2H, $J=8.84 \mathrm{~Hz}, 2.12 \mathrm{~Hz}$, biphenyl), 7.01 (dd, 2H, $J=$ $8.64 \mathrm{~Hz}, 2.11 \mathrm{~Hz}$, biphenyl), $4.14\left(\mathrm{~m}, 1 \mathrm{H},-\mathrm{OC}_{\underline{a}} \mathrm{H}_{\mathrm{b}^{-}}\right), 4.10\left(\mathrm{~m}, 1 \mathrm{H},-\mathrm{OCH}_{\mathrm{a}} \underline{\mathrm{H}}^{-}\right), 1.88(\mathrm{~m}, 1 \mathrm{H},-$ $\left.\mathrm{OCH}_{2} \mathrm{CH} \mathrm{CH}_{3} \mathrm{CH}_{2}-\right), 1.57$ (m, 1H, $\left.\mathrm{CH}\left(\mathrm{CH}_{3}\right)_{2} \mathrm{CH}_{2}-\right), 1.30\left(\mathrm{~m}, 6 \mathrm{H},-\left(\mathrm{CH}_{2}\right)_{3}\right), 0.98(\mathrm{~d}, 3 \mathrm{H}, J=6.52$ $\left.\mathrm{Hz}, \underline{\mathrm{CH}}_{3} \mathrm{CH}-\right), 0.90$ (d, $\left.6 \mathrm{H}, J=6.64 \mathrm{~Hz},\left(\mathrm{C}_{\underline{3}}\right)_{2} \mathrm{CH}-\right)$.

\section{4-(4-\{[(2R)-2,6-Dimethylheptyl]oxy\}phenyl) benzoic acid (7a)}

Compound 6a (1.25 g, $3.84 \mathrm{mmol})$ and lithium hydroxide monohydrate (1.607 g, $38.4 \mathrm{mmol})$ were dissolved in a $30 \mathrm{~mL}$ ethanol / $30 \mathrm{~mL}$ water mixture. The hydrolysis reaction was refluxed for 20 hours at $80{ }^{\circ} \mathrm{C}$. High concentration hydrochloric acid $(\mathrm{HCl}, \sim 37 \%)$ was drop by drop added to the mixture to adjust the $\mathrm{pH}$ reached $\mathrm{pH}=2$. The resulting material was collected by vacuum filtration, washed with water three times and dried in a vacuum oven overnight to obtain a white solid (1.22 g, 95\%). ${ }^{1} \mathrm{H}$ NMR $\left(\mathrm{CDCl}_{3}, 400 \mathrm{MHz}, \delta / \mathrm{ppm}\right): \delta 8.10(\mathrm{dd}, 2 \mathrm{H}, J=8.52 \mathrm{~Hz}$, $4.88 \mathrm{~Hz}$, biphenyl), 7.64 (dd, 2H, $J=8.80 \mathrm{~Hz}, 2.10 \mathrm{~Hz}$, biphenyl), 7.59 (dd, 2H, $J=8.84 \mathrm{~Hz}$, $2.12 \mathrm{~Hz}$, biphenyl), 7.01 (dd, $2 \mathrm{H}, J=8.64 \mathrm{~Hz}, 2.11 \mathrm{~Hz}$, biphenyl), 4.14 (m, 1H, $\left.-\mathrm{OC}_{\underline{a}} \mathrm{H}_{\mathrm{b}^{-}}\right), 4.10$ $\left(\mathrm{m}, 1 \mathrm{H},-\mathrm{OCH}_{\mathrm{a}} \underline{\mathrm{H}}_{\underline{\mathrm{b}}}\right)$ ), $3.95\left(\mathrm{~s}, 3 \mathrm{H},-\mathrm{COOC}_{\underline{3}}\right), 1.88\left(\mathrm{~m}, 1 \mathrm{H},-\mathrm{OCH}_{2} \mathrm{CHCH}_{3} \mathrm{CH}_{2}-\right), 1.57(\mathrm{~m}, 1 \mathrm{H}$, $\left.\mathrm{C} \underline{\mathrm{H}}\left(\mathrm{CH}_{3}\right)_{2} \mathrm{CH}_{2}-\right), 1.30\left(\mathrm{~m}, 6 \mathrm{H},-\left(\mathrm{CH}_{2}\right)_{3}\right), 0.98\left(\mathrm{~d}, 3 \mathrm{H}, \mathrm{J}=6.52 \mathrm{~Hz}, \mathrm{CH}_{3} \mathrm{CH}-\right), 0.90$ (d, $6 \mathrm{H}, J=6.64$ $\left.\mathrm{Hz},\left(\mathrm{CH}_{3}\right)_{2} \mathrm{CH}-\right)$.

\section{4-[3-(Oxan-2-yloxy)phenyl]phenyl 4-(4-\{[(2R)-2,7-dimethyloctyl]oxy\}phenyl) benzoate (8a)}

In a $\mathrm{N}_{2}$-purged two-necked round flask, 4'-hydroxy-3-tetrahydropyranyloxybiphenyl ${ }^{2-4}$ (281.2 $\mathrm{mg}, 1.04 \mathrm{mmol}$ ), compound $7 \mathbf{a}(220.7 \mathrm{mg}, 0.68 \mathrm{mmol})$, and 4-(dimethylamino)pyridine (DMAP) (279.6 mg, $2.28 \mathrm{mmol})$ was added to dichloromethane $(10 \mathrm{~mL})$ and THF $(10 \mathrm{~mL})$ mixture. After stirring for 20 minutes, 1-ethyl-3-(3-dimethylaminopropyl) carbodiimide (EDCI, $600 \mathrm{mg}, 3.12$ mmol) was added to this mixture. The reaction was stirred at room temperature under $\mathrm{N}_{2}$ for 24 hours and followed by TLC. The mixture was washed with saturated $\mathrm{NaCl}$ solution and extracted by $\mathrm{CHCl}_{3}$, and then dried by anhydrous $\mathrm{Na}_{2} \mathrm{SO}_{4}$. The product was concentrated by rotary evaporation and purified by column chromatography with $\mathrm{CHCl}_{3}$, then dried in a vacuum oven overnight to yield the final white solid (102 mg, 17\%). ${ }^{1} \mathrm{H}$ NMR $\left(\mathrm{CDCl}_{3}, 400 \mathrm{MHz}, \delta / \mathrm{ppm}\right): \delta$ 8.29 (dt, 2H, $J=8.56,1.08 \mathrm{~Hz}$, biphenyl), 7.73 (dt, 2H, $J=8.40,0.80 \mathrm{~Hz}$, biphenyl), 7.69 (dt, 
2H, $J=6.68,2.12 \mathrm{~Hz}$, biphenyl), 7.64 (dt, 2H, $J=6.64,2.08 \mathrm{~Hz}$, biphenyl), 7.40 (t, $1 \mathrm{H}, J=7.88$ $\mathrm{Hz}$, biphenyl), 7.35-7.32 (m, 3H, biphenyl), 7.26 (dt, 1H, $J=7.50,1.20 \mathrm{~Hz}$, biphenyl), 7.12-7.09 (m, 1H, biphenyl), 7.05 (dt, 2H, $J=8.88,2.91 \mathrm{~Hz}$, biphenyl), 5.53 (t, 1H, $J=3.32 \mathrm{~Hz},-\mathrm{OC} \underline{\mathrm{H}}$ ( $\left.\mathrm{CH}_{2}-\right)-\mathrm{O}-$, THP), 4.20-4.17 (m, 1H, -OCH $\left.\underline{\mathrm{H}}_{\underline{a}} \mathrm{H}_{\mathrm{b}}-\mathrm{CH}-\mathrm{CH}_{2}\left(\mathrm{CH}_{3}\right)\right), 4.17-4.13\left(\mathrm{~m}, 1 \mathrm{H},-\mathrm{OCH}_{\vec{a}} \underline{\mathrm{H}_{\underline{b}}}-\mathrm{CH}-\right.$ $\left.\mathrm{CH}_{2}\left(\mathrm{CH}_{3}\right)\right)$, 4.05-3.97 (m, 1H, -O- $\underline{\mathrm{H}}_{\underline{a}} \mathrm{H}_{\mathrm{b}}-\mathrm{CH}_{2}-$, THP), 3.70-3.65 (m, $\left.1 \mathrm{H},-\mathrm{O}-\mathrm{CH}_{a} \underline{\mathrm{H}_{b}}-\mathrm{CH}_{2^{-}}, \mathrm{THP}\right)$, 2.14-1.86 (m, 3+2H, THP, $\left.-\mathrm{OC}_{2} \mathrm{H}_{4}-\mathrm{C}_{2}-\mathrm{C}_{5} \mathrm{H}_{11}\right), 1.75-1.62$ (m, 3H, THP), 1.42-1.19 (m, 6H,$\left.\mathrm{CHCH}_{3} \mathrm{CH}_{2}-\left(\mathrm{CH}_{2}\right)_{3}-\mathrm{CH}\left(\mathrm{CH}_{3}\right)_{2}\right), 1.02-0.92\left(\mathrm{~m}, 6 \mathrm{H}+3 \mathrm{H},-\left(\mathrm{CH}_{3}\right)_{2} \mathrm{CH}-, \mathrm{CH}_{2}-\mathrm{CH}-\right)$.

\section{4-(3-Hydroxyphenyl)phenyl 4-(4-\{[(2R)-2,7-dimethyloctyl]oxy\}phenyl) benzoate (9a)}

Compound 8a (51.4 mg, $0.09 \mathrm{mmol})$ was dissolved in DCM $(13 \mathrm{~mL})$, then added high concentrated $\mathrm{HCl}(162 \mu \mathrm{L})$ to this mixture. The whole mixture was stirred at room temperature for 12 hours. After that, $\mathrm{NaHCO}_{3}(0.50 \mathrm{~g})$ and water $(20 \mathrm{~mL})$ were added. The mixture was extracted with $\mathrm{CHCl}_{3}$ and dried by anhydrous $\mathrm{Na}_{2} \mathrm{SO}_{4}$. The filtrate was concentrated by rotary evaporation, the residue was purified by column chromatography $\left(\mathrm{CHCl}_{3} / \mathrm{MeOH}=10 / 0.5\right)$ to yield a white solid (56 mg, 60\%). ${ }^{1} \mathrm{H}$ NMR $\left(\mathrm{CDCl}_{3}, 400 \mathrm{MHz}, \delta / \mathrm{ppm}\right): \delta 8.28(\mathrm{dt}, 2 \mathrm{H}, J=8.80$ $\mathrm{Hz}, 2.0 \mathrm{~Hz}$, biphenyl), 7.73 (dt, 2H, $J=8.0 \mathrm{HZ}, 2.24 \mathrm{~Hz}$, biphenyl), 7.66-7.61 (m, 4H, biphenyl), 7.36-7.28 (m, 3H, biphenyl), 7.20-7.18 (m, H, biphenyl), 7.08-7.03 (m, 3H, biphenyl), 6.87-6.84 (m, 1H, biphenyl), 5.01 (s, H, -OH), 4.20-4.17 (m, 1H, $\left.-\mathrm{OC}_{\underline{a}} \mathrm{H}_{\mathrm{b}}-\mathrm{CH}-\mathrm{CH}_{2}\left(\mathrm{CH}_{3}\right)\right)$, 4.17-4.13 (m, $\left.1 \mathrm{H},-\mathrm{OCH}_{a} \underline{\mathrm{H}_{b}}-\mathrm{CH}-\mathrm{CH}_{2}\left(\mathrm{CH}_{3}\right)\right), 1.93-1.85\left(\mathrm{~m}, 1 \mathrm{H},-\mathrm{OCH}_{2} \underline{\mathrm{CHCH}}_{3} \mathrm{CH}_{2}-\right)$, 1.74-1.64 (m, H, $\left.\left(\mathrm{CH}_{3}\right)_{2} \mathrm{CHCH}_{2}-\right), 1.43-1.18$ (m, 6H, $\left.-\left(\mathrm{C}_{2}\right)_{3}-\right), 1.02-0.90\left(\mathrm{~m}, 6 \mathrm{H}+3 \mathrm{H},-\left(\mathrm{C}_{3}\right)_{2}-\mathrm{CH}-, \mathrm{CH}_{3}-\mathrm{CH}-\right)$.

\section{Methyl 4-[2-(S)-nonan-2-yloxy]biphenyl-4'-carboxylate (6b)}

In a two-neck $\mathrm{N}_{2}$ purged round bottom flask, methyl 4'-hydroxy(1,1'-biphenyl)-4-carboxylate (267.35 mg, $1.17 \mathrm{mmol})$, triphenylphosphine (TPP) (403.35 mg, $1.525 \mathrm{mmol}$ ) and (2R)-decan-2ol $(291.87 \mu \mathrm{L}, 1.525 \mathrm{mmol})$ were dissolved in $5 \mathrm{~mL}$ of THF. To this, a solution of $1 \mathrm{~mL}$ THF and diisopropyl azodicarboxylate (DIAD) $(297 \mu \mathrm{L}, 1.525 \mathrm{mmol})$ was added dropwise while solution was stirred. The mixture was placed in ice bath and left to stir for 3 hours. The result was washed with saturated $\mathrm{NaCl}$ solution, extracted with chloroform and dried using sodium sulfate. The solution was filtered, concentrated using rotary evaporation and purified using column chromatography with chloroform. The result was a white solid (155 mg, 36\%). ${ }^{1} \mathrm{H}$ NMR ( $\left.\mathrm{CDCl}_{3}, 400 \mathrm{MHz}, \delta / \mathrm{ppm}\right): \delta 8.10$ (d, 2H, $J=6 \mathrm{~Hz}$, biphenyl), 7.65 (d, 2H, $J=8 \mathrm{~Hz}$, biphenyl), 
7.58 (d, 2H, $J=8 \mathrm{~Hz}$, biphenyl), 7.00 (d, 2H, $J=4 \mathrm{~Hz}$, biphenyl), 4.40-4.48 (m, 1H, -O$\left.\mathrm{C} \underline{\mathrm{H}}\left(\mathrm{CH}_{3}\right)-\mathrm{CH}_{2}-\right), 3.96$ (s, 3H, -O-CH3), 1.31-1.84 (m, 17H, $\left.-\mathrm{CH}_{2}-\mathrm{CH}_{2}-\right), 0.92$ (t, 3H, J = $8 \mathrm{~Hz}$, $\left.\mathrm{C}_{3}-\mathrm{CH}_{2}-\right) .{ }^{13} \mathrm{C} \mathrm{NMR}\left(\mathrm{CDCl}_{3}, 400 \mathrm{MHz}, \delta / \mathrm{ppm}\right): \delta 167.23,158.64,145.36,132.02,130.10$, $128.36,126.40,116.16,74.03,52.04,36.49,31.89,29.63,29.57,29.27,25.58,22.68,19.77$, 14.11 .

\section{4-[2-(S)-Nonan-2-yloxy]biphenyl-4'-carboxylic acid (7b)}

Methyl 4-[2-(S)-nonan-2-yloxy]biphenyl-4'-carboxylate $\mathbf{6 b}$ was dissolved with lithium hydroxide monohydrate $(176.5 \mathrm{mg}, 4.206 \mathrm{mmol})$ in $15 \mathrm{~mL}$ of ethanol and $15 \mathrm{~mL}$ of water and refluxed for 16 hours. The solvent was removed through rotary evaporation and hydrochloric acid was added dropwise until the $\mathrm{pH}$ was 2 . The solution was vacuum filtered to collect the white precipitate and washed with water (134 mg, 90\%). ${ }^{1} \mathrm{H}$ NMR (DMSO, $\left.400 \mathrm{MHz}, \delta / \mathrm{ppm}\right): \delta$ 7.98 (d, 2H, $J=8 \mathrm{~Hz}$, biphenyl), 7.70 (d, 2H, $J=8 \mathrm{~Hz}$, biphenyl), 7.65 (d, 2H, $J=8 \mathrm{~Hz}$, biphenyl), 7.01 (d, 2H, $J=8 \mathrm{~Hz}$, biphenyl), 4.46-4.53 (m, 1H, - $\left.\mathrm{O}-\mathrm{C} \underline{\mathrm{H}}\left(\mathrm{CH}_{3}\right)-\mathrm{CH}_{2}-\right), 1.33-1.83$ (m, $\left.17 \mathrm{H}, \mathrm{CH}-\underline{\mathrm{H}}_{3},-\mathrm{CH}_{2}-\mathrm{CH}_{2}-\right), 0.86\left(\mathrm{t}, 3 \mathrm{H}, \mathrm{J}=8 \mathrm{~Hz}, \mathrm{CH}_{3}-\mathrm{CH}_{2}-\right)$.

\section{4'-[4-(4-[2-(S)-Nonan-2-yloxy]biphenyl]-3-tetrahydropyranyloxybiphenyl (8b)}

In $\mathrm{N}_{2}$ purged flask, 4'-hydroxy-3-tetrahydropyranyloxybiphenyl ${ }^{2-4}$ (77.9 mg, $0.288 \mathrm{mmol}$ ), 4-[2(S)-nonan-2-yloxy]biphenyl-4'-carboxylic acid 7b (133.7 mg, $0.377 \mathrm{mmol})$, and 4-(dimethylamino)pyridine (DMAP) (77.26 mg, $0.632 \mathrm{mmol})$ were dissolved in $5 \mathrm{~mL}$ dichloromethane and $5 \mathrm{~mL}$ THF and stirred. After 5 minutes, EDCI (163.1 mg, $0.865 \mathrm{mmol})$ was dissolved in small amount of dichloromethane and added to the round bottom flask. The reaction was left to stir at room temperature for 24 hours under nitrogen atmosphere. The mixture was examined using TLC, washed with saturated solution of sodium chloride, extracted using chloroform and dried using sodium sulfate. The solvent was collected using filtration and concentrated by rotary evaporation. The crude product was purified by column chromatography with chloroform. The result is a white solid (157 mg, 98\%). ${ }^{1} \mathrm{H} \mathrm{NMR}\left(\mathrm{CDCl}_{3}, 400 \mathrm{MHz}, \delta / \mathrm{ppm}\right): \delta 8.29(\mathrm{~d}, 2 \mathrm{H}, J=$ 8Hz, biphenyl), 7.74 (d, 2H, $J=8 \mathrm{~Hz}$, biphenyl), 7.685 (d, 2H, $J=12 \mathrm{~Hz}$, biphenyl), 7.63 (d, 2H, $J=8 \mathrm{~Hz}$, biphenyl), 7.37 (t, 1H, $J=8 \mathrm{~Hz}$, biphenyl), 7.315 (d, 2H, $J=12 \mathrm{~Hz}$, biphenyl), 7.28 (d, $1 \mathrm{H}, J=8 \mathrm{~Hz}$, biphenyl), 7.26 (s, 1H, biphenyl), 7.115 (d, 1H, $J=4 \mathrm{~Hz}$, biphenyl), 7.03 (d, 2H, $J$ $=8 \mathrm{~Hz}$, biphenyl), $5.54(\mathrm{~s}, 1 \mathrm{H}, \mathrm{THP}), 4.42-4.50\left(\mathrm{~m}, 1 \mathrm{H},-\mathrm{O}-\mathrm{CH}\left(\mathrm{CH}_{3}\right)-\mathrm{CH}_{2}-\right), 3.99-4.02(\mathrm{~m}, 1 \mathrm{H},-$ 
O- $\underline{\mathrm{H}}_{\mathrm{a}} \mathrm{CH}_{\mathrm{b}}-\mathrm{CH}_{2}-$, THP), 3.66-3.69 (m, $\left.1 \mathrm{H},-\mathrm{O}-\mathrm{CH}_{\mathrm{a}} \underline{\mathrm{CH}}_{\mathrm{b}}-\mathrm{CH}_{2}, \mathrm{THP}\right), 2.03-2.12$ (m, $1 \mathrm{H}, \mathrm{THP}$ ), 1.91-1.95 (m, 2H, THP), 1.59-1.85 (m, 3H, CH-CH $\left.{ }_{3}\right), 1.41-1.55$ (m, 3H, THP), 1.30-1.39 (m,

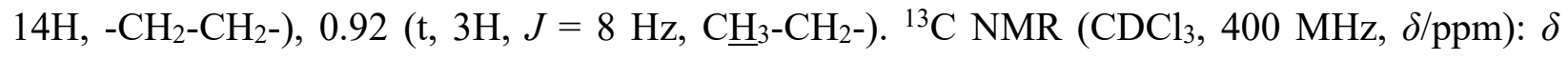
$165.23,158.74,157.52,150.51,146.06,141.83,138.81,130.78,129.77,128.47,128.28,126.60$, $121.99,120.50,116.19,115.50,96.46,74.03,62.11,36.49,31.91,30.44,29.75,29.65,29.60$, $29.30,25.61,25.26,22.71,19.79,18.83,14.16$.

\section{4'-[4-(4-[2-(S)-Nonan-2-yloxy]biphenyl)]-3-hydroxybiphenyl (9b)}

4'-[4-(4-[2-(S)-nonan-2-yloxy]biphenyl]-3-tetrahydropyranyloxybiphenyl $\mathbf{8 b}$ was dissolved in $6.5 \mathrm{~mL}$ dichloromethane and hydrochloric acid $(33 \%, 243 \mu \mathrm{L})$ was added. The solution was left to stir at room temperature for 12 hours. A solution composed of sodium bicarbonate $(0.25 \mathrm{~g})$ and $10 \mathrm{~mL}$ of water was then added and the solid was filtered off and washed with chloroform. The filtrate was dried using sodium sulfate, filtered and concentrated using rotary evaporation. Purification was performed using column chromatography with a chloroform/methanol (10/0.5) system. The product was a white solid (69 mg, 47.15\%). ${ }^{1} \mathrm{H}$ NMR $\left(\mathrm{CDCl}_{3}, 400 \mathrm{MHz}, \delta / \mathrm{ppm}\right): \delta$ 8.28 (d, 2H, $J=12 \mathrm{~Hz}$, biphenyl), 7.73 (d, 2H, $J=8 \mathrm{~Hz}$, biphenyl), 7.64 (d, $2 \mathrm{H}, J=2 \mathrm{~Hz}$, biphenyl), 7.62 (d, 2H, $J=4 \mathrm{~Hz}$, biphenyl), 7.33 (t, 1H, $J=8 \mathrm{~Hz}$, biphenyl), 7.29 (s, 2H, biphenyl), 7.18 (d, 1H, $J=8 \mathrm{~Hz}$, biphenyl), 7.07 (s, 1H, biphenyl), 7.03 (d, $1 \mathrm{H}, J=8 \mathrm{~Hz}$, biphenyl), 6.86 (d, $2 \mathrm{H}, J=8 \mathrm{~Hz}$, biphenyl), 4.42-4.49 (m, 1H, -O-C $\left.\underline{\mathrm{H}}\left(\mathrm{CH}_{3}\right)-\mathrm{CH}_{2}-\right)$, 1.29-1.54 (m, $\left.3 \mathrm{H}, \mathrm{CH}-\mathrm{CH}_{3}\right), 1.30-1.39$ (m, $\left.14 \mathrm{H},-\mathrm{CH}_{2}-\mathrm{CH}_{2}-\right), 0.93$ (t, 3H, $J=8 \mathrm{~Hz}, \mathrm{CH}_{3}-\mathrm{CH}_{2}-$ ).

\section{4'-[4-(4-Decyloxy)biphenyloxy]-3-tetrahydropyranyloxybiphenyl (8d)}

$\mathbf{8 d}$ was synthesized following the procedure outlined for compound $\mathbf{8 b}$. Quantities: 4'-hydroxy3-tetrahydropyranyloxybiphenyl ${ }^{2-4}(77.9 \mathrm{mg}, 0.288 \mathrm{mmol})$, 4-decyloxy biphenyl-4'-carboxylic acid (133.7 mg, $0.377 \mathrm{mmol}$ ), DMAP (77.26 mg, $0.632 \mathrm{mmol}$ ), EDCI (163.14 mg, $0.865 \mathrm{mmol})$. Yield: $130 \mathrm{mg}(81.25 \%) .{ }^{1} \mathrm{H} \mathrm{NMR}\left(\mathrm{CDCl}_{3}, 400 \mathrm{MHz}, \delta / \mathrm{ppm}\right): \delta 8.29(\mathrm{~d}, 2 \mathrm{H}, J=8 \mathrm{~Hz}$, biphenyl), 7.735 (d, 2H, $J=12 \mathrm{~Hz}$, biphenyl), 7.66 (d, 2H, $J=8 \mathrm{~Hz}$, biphenyl), 7.39 (d, 2H, $J=$ $8 \mathrm{~Hz}$, biphenyl), 7.32 (t, $1 \mathrm{H}, J=8 \mathrm{~Hz}$, biphenyl), 7.25 (s, 2H, biphenyl), 7.115 (d, $1 \mathrm{H}, J=4 \mathrm{~Hz}$, biphenyl), 7.09 (s, $1 \mathrm{H}$, biphenyl), 7.04 (d, $1 \mathrm{H}, J=8 \mathrm{~Hz}$, biphenyl), 5.54 (t, $1 \mathrm{H}, J=4 \mathrm{~Hz}$, biphenyl), 4.05 (t, $2 \mathrm{H}, J=8 \mathrm{~Hz},-\mathrm{O}-\underline{\mathrm{C}}_{2}-\mathrm{CH}_{2}-$ ), 3.96-3.99 (m, $1 \mathrm{H},-\mathrm{O}-\mathrm{C}_{\mathrm{H}} \mathrm{CH}_{\mathrm{b}}-\mathrm{CH}_{2}$, THP), 3.65-3.40 (m, $\left.1 \mathrm{H},-\mathrm{O}-\mathrm{CH}_{\mathrm{a}} \mathrm{C}_{\mathrm{b}}-\mathrm{CH}_{2}, \mathrm{THP}\right), 2.03-2.14$ (m, $\left.1 \mathrm{H}, \mathrm{THP}\right), 1.91-1.95$ (m, 2H, THP), 
1.82-1.89 (m, 1H, THP), 1.63-1.79 (m, 3H, THP), 1.30-1.55 (m, 15H, $\left.-\mathrm{CH}_{2}-\mathrm{CH}_{2}-\right), 0.93$ (t, 3H, $J$ $\left.=8 \mathrm{~Hz}, \mathrm{CH}_{3}-\mathrm{CH}_{2}-\right) .{ }^{13} \mathrm{C} \mathrm{NMR}\left(\mathrm{CDCl}_{3}, 400 \mathrm{MHz}, \delta / \mathrm{ppm}\right): \delta 165.32,159.86,157.78,151.35$, 145.62, 141.98, 138.46, 132.06, 130.78, 129.87, 128.42, 128.28, 127.36, 126.62, 121.99, 120.64, $116.02,115.01,96.41,68.87,62.11,32.45,30.44,29.61,29.45,29.37,29.29,26.09,25.26,23.12$, $18.83,14.36$.

\section{4'-[4-(4-Decyloxy)biphenyloxy]-3-hydroxybiphenyl (9d)}

9d was synthesized following the procedure outlined for compound 9b. Quantities: 4'-[4-(4decyloxy)biphenyloxy]-3-tetrahydropyranyloxybiphenyl $\mathbf{8 d}$ (130 mg, $0.2318 \mathrm{mmol})$. Yield: 73 mg (50\%). ${ }^{1} \mathrm{H}$ NMR $\left(\mathrm{CDCl}_{3}, 400 \mathrm{MHz}, \delta / \mathrm{ppm}\right): \delta 8.28$ (d, $2 \mathrm{H}, J=8 \mathrm{~Hz}$, biphenyl), 7.73 (d, $2 \mathrm{H}$, $J=8 \mathrm{~Hz}$, biphenyl), 7.65 (d, 2H, $J=8 \mathrm{~Hz}$, biphenyl), 7.63 (d, 2H, $J=8 \mathrm{~Hz}$, biphenyl), 7.34 (t, $1 \mathrm{H}, J=4 \mathrm{~Hz}$, biphenyl), 7.34 (d, 2H, $J=8 \mathrm{~Hz}$, biphenyl), 7.20 (d, 1H, $J=4 \mathrm{~Hz}$, biphenyl), 7.09 (s, 1H, biphenyl), 7.04 (d, 2H, $J=8 \mathrm{~Hz}$, biphenyl), 6.86 (d, 2H, $J=8 \mathrm{~Hz}$, biphenyl), 4.97 (s, 1H, $-\mathrm{OH}$ ), 4.05 (t, $\left.2 \mathrm{H}, J=6 \mathrm{~Hz},-\mathrm{O}-\mathrm{C}_{2}-\mathrm{CH}_{2}-\right), 1.81-1.88$ (m, 2H, -O- $\mathrm{CH}_{2}-\mathrm{C}_{2}-$ ), 1.49-1.59 (m, 4H, $\left.-\mathrm{CH}_{2}-\mathrm{CH}_{2}-\right), 1.28-1.40\left(\mathrm{~m}, 10 \mathrm{H},-\mathrm{CH}_{2}-\mathrm{CH}_{2}-\right), 0.90\left(\mathrm{t}, 3 \mathrm{H}, J=2 \mathrm{~Hz}, \mathrm{CH}_{3}-\mathrm{CH}_{2}-\right)$.

\section{Final products}

\section{4'-[4-(4'-Decyloxy)biphenyl)]-3-[4-(4'-[2-(S)-nonan-2-yloxy]biphenyl)]-biphenyl, 1}

In a $\mathrm{N}_{2}$-purged round bottom flask, 3,4'-dihydroxybiphenyl 4'-[4-(4'-decyloxy)biphenyloxy]-3hydroxybiphenyl 9d (73 mg, $0.1397 \mathrm{mmol})$, 4-[2-(S)-nonan-2-yloxy]biphenyl-4'-carboxylic acid $7 \mathbf{b}(50.78 \mathrm{mg}, 0.15 \mathrm{mmol})$, and DMAP $(18.33 \mathrm{mg}, 0.15 \mathrm{mmol})$ were dissolved in a mixture of dichloromethane $(10 \mathrm{~mL})$ and THF $(10 \mathrm{~mL})$ and stirred for 10 minutes. To this mixture was added EDCI (28.75 mg, $0.15 \mathrm{mmol})$. The reaction was kept at room temperature for 24 hours. The resulting mixture was washed with saturated $\mathrm{NaCl}$ solution, extracted with $\mathrm{CHCl}_{3}$, and then dried by anhydrous $\mathrm{Na}_{2} \mathrm{SO}_{4}$. The residue was examined by TLC and concentrated by rotary evaporation. The residue was purified by column chromatography with $\mathrm{CHCl}_{3}$ to get a white solid final product (115 mg, 95\%). ${ }^{1} \mathrm{H}$ NMR (DMSO, $400 \mathrm{MHz}, \delta / \mathrm{ppm}$ ): $\delta 8.31$ (d, $4 \mathrm{H}, J=8 \mathrm{~Hz}$, biphenyl), 7.74 (d, 6H, $J=8 \mathrm{~Hz}$, biphenyl), 7.64 (d, 4H, $J=8 \mathrm{~Hz}$, biphenyl), 7.55 (d, 2H, $J=2$ $\mathrm{Hz}$, biphenyl), 7.52 (d, 1H, $J=8 \mathrm{~Hz}$, biphenyl), 7.36 (d, 2H, $J=6 \mathrm{~Hz}$, biphenyl), 7.29 (d, 1H, $J=$ $8 \mathrm{~Hz}$, biphenyl), 7.04 (d, 4H, $J=8 \mathrm{~Hz}$, biphenyl), 4.45-4.49 (m, 1H, -O-C $\left.\underline{\mathrm{H}}\left(\mathrm{CH}_{3}\right)-\mathrm{CH}_{2}-\right), 4.05$ (t, $\left.2 \mathrm{H}, J=8 \mathrm{~Hz},-\mathrm{O}-\mathrm{C}_{2}-\mathrm{CH}_{2}-\right), 1.79-1.90\left(\mathrm{~m}, 3 \mathrm{H},-\mathrm{CH}\left(\mathrm{C}_{3}\right)-\right), 1.61-1.68\left(\mathrm{~m}, 4 \mathrm{H},-\mathrm{CH}_{2}-\mathrm{C}_{2}-\right)$, 
1.32-1.55 (m, 26H, - $\left.\mathrm{CH}_{2}-\mathrm{CH}_{2}-\right), 0.93\left(\mathrm{t}, 6 \mathrm{H}, \mathrm{CH}_{3}\right){ }^{13} \mathrm{C} \mathrm{NMR}\left(\mathrm{CDCl}_{3}, 400 \mathrm{MHz}, \delta / \mathrm{ppm}\right): \delta$ $164.86,159.87,158.76,151.50,150.79,146.06,142.31$, 137.96, 131.92, 130.80, 129.89, 129.06, $128.49,128.43,128.33,127.43,126.62,124.36,122.18,120.41,116.21,115.02,74.03,68.81$, $36.52,31.94,29.64,29.33,26.11,25.63,22.76,19.81,14.20$. Elemental analysis: calculated for $\mathrm{C}_{58} \mathrm{H}_{66} \mathrm{O}_{6}: \mathrm{C}, 81.08 ; \mathrm{H}, 7.74$; Found: C, 80.92; H, 7.54.

\section{4'-[4-(4'-[2-(S)-Nonan-2-yloxy]biphenyl)]-3-[4-(4'-decyloxy)biphenyl)]-biphenyl, 2}

Compound 2 was synthesized according to the procedure outlined for compound 1. Quantities: 4'-[4-(4'-[2-(S)-nonan-2-yloxy]biphenyl)]-3-hydroxybiphenyl 9b (69 mg, $0.132 \mathrm{mmol})$, 4-ndecyloxybiphyl-4'-carboxylic acid $7 \mathbf{d}$ (50.78 $\mathrm{mg}, 0.15 \mathrm{mmol})$, DMAP (18.33 mg, $0.15 \mathrm{mmol})$ and EDCI (28.75 mg, $0.15 \mathrm{mmol})$. The final product was a white solid $(95 \mathrm{mg}, 84 \%) .{ }^{1} \mathrm{H}$ NMR $\left(\mathrm{CDCl}_{3}, 400 \mathrm{MHz}, \delta / \mathrm{ppm}\right): \delta 8.30$ (dd, $4 \mathrm{H}, J=8 \mathrm{~Hz}$, biphenyl), 7.72 (d, $6 \mathrm{H}, J=8 \mathrm{~Hz}$, biphenyl), 7.64 (d, 4H, $J=8 \mathrm{~Hz}$, biphenyl), 7.54 (d, 2H, $J=2 \mathrm{~Hz}$, biphenyl), 7.50 (s, 1H, biphenyl), 7.35 (d, $2 \mathrm{H}, J=8 \mathrm{~Hz}$, biphenyl), 7.03 (d, 1H, $J=8 \mathrm{~Hz}$, biphenyl), 7.02 (d, 4H, $J=8 \mathrm{~Hz}$, biphenyl), 4.42$4.49\left(\mathrm{~m}, 1 \mathrm{H},-\mathrm{O}-\mathrm{CH}\left(\mathrm{CH}_{3}\right)-\mathrm{CH}_{2}-\right), 4.05\left(\mathrm{t}, 2 \mathrm{H}, J=8 \mathrm{~Hz},-\mathrm{O}-\mathrm{C}_{2}-\mathrm{CH}_{2}-\right), 1.76-1.88(\mathrm{~m}, 3 \mathrm{H}$, $\left.\mathrm{CH}\left(\mathrm{C}_{3}\right)-\right), 1.62-1.66$ (m, 4H, $\left.\mathrm{CH}_{2}\right), 1.38-1.55\left(\mathrm{~m}, 26 \mathrm{H}, \mathrm{CH}_{2}\right), 0.91$ (t, $\left.6 \mathrm{H}, J=8 \mathrm{~Hz}, \underline{\mathrm{C}}_{3}\right) .{ }^{13} \mathrm{C}$ NMR $\left(\mathrm{CDCl}_{3}, 400 \mathrm{MHz}, \delta / \mathrm{ppm}\right): \delta 165.16,159.63,158.77,151.49,150.80,146.09,142.10$, $137.98,130.77,129.85,128.45,128.41,126.60,124.61,122.14,120.68,120.52,116.22,115.03$, $74.06,68.20,36.49,31.92,31.89,29.72,29.63,29.58,29.42,29.34,29.28,26.07,25.58,22.70$, 19.78, 14.13. Elemental analysis: calculated for $\mathrm{C}_{58} \mathrm{H}_{66} \mathrm{O}_{6}: \mathrm{C}, 81.08 ; \mathrm{H}, 7.74$; Found: $\mathrm{C}, 81.01 ; \mathrm{H}$, 7.78.

4'-((4'-(((R)-2,6-Dimethylheptyl)oxy)-[1,1'-biphenyl]-4-carbonyl)oxy)-[1,1'-biphenyl]-3-yl4'-(( $R$ )-2,6-dimethylheptyl)oxy)-[1,1'-biphenyl]-4-carboxylate, 3

Compound 3 was synthesized according to the procedure outlined for compound 1. Quantities: 3,4'-dihydroxybiphenyl (88 mg, $0.47 \mathrm{mmol}),(R)-4$ '-((6-methylheptan-2-yl)oxy)-[1,1'-biphenyl]4-carboxylic acid 7a (200 mg, $0.608 \mathrm{mmol}$ ), 4-(dimethylamino) pyridine (DMAP) (35 mg, 0.29 $\mathrm{mmol})$, and EDCI (74 mg, $0.385 \mathrm{mmol})$. The final product was a white solid (100 mg, 29\%). ${ }^{1} \mathrm{H}$ NMR ( $\left.\mathrm{CDCl}_{3}, 400 \mathrm{MHz}, \delta / \mathrm{ppm}\right): \delta 8.32(\mathrm{dd}, 4 \mathrm{H}, J=8.44 \mathrm{~Hz}, 1.68), 7.70-7.76$ (m, 6H, biphenyl), 7.65 (dt, $4 \mathrm{H}, J=8.80 \mathrm{~Hz}, 3.04 \mathrm{~Hz}$, biphenyl), 7.55 (dt, $2 \mathrm{H}, J=1.72 \mathrm{~Hz}, 8.64 \mathrm{~Hz}$, biphenyl), 7.51 (quin, 1H, biphenyl), 7.35 (dd, 2H, $6.40 \mathrm{~Hz}, 1.60 \mathrm{~Hz}$ biphenyl), 7.29 (m, 1H, biphenyl), 7.05 (dt, 
$4 \mathrm{H}, J=8.80 \mathrm{~Hz}, 3.3 \mathrm{~Hz}$, biphenyl), 4.03-4.10 (m, 4H, $-\mathrm{OCH}_{\underline{a}} \underline{\mathrm{H}_{\mathrm{b}}}$ ), 1.90-1.95 (sex, $2 \mathrm{H}$, $\left.\mathrm{OCH}_{2} \mathrm{CH}\left(\mathrm{CH}_{3}\right)-\right), 1.58-1.76\left(\mathrm{~m}, 2 \mathrm{H},-\mathrm{CH}\left(\mathrm{CH}_{3}\right)_{2}\right), 1.25-1.64(\mathrm{~m}, 17 \mathrm{H}), 0.95-1.01(\mathrm{~m}, 3 \mathrm{H}+9 \mathrm{H},-$

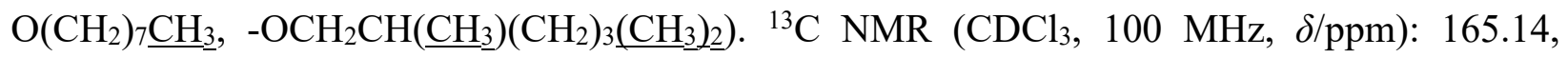
165.11, 159.66, 151.54, 150.83, 146.05, 142.08, 137.96, 131.96, 130.80, 129.88, 128.43, 127.51, $126.63,124.61,122.18,120.72,120.53,115.07,77.12$, 76.80, 66.52, 39.32, 37.37, 36.25, 29.94, 28.04, 24.74, 22.79, 22.69, 19.74. Elemental analysis: calculated for $\mathrm{C}_{56} \mathrm{H}_{62} \mathrm{O}_{6}: \mathrm{C}, 80.93 ; \mathrm{H}, 7.52$; Found: C, 80.85; H, 7.48.

(R)-3'-((4'-((2,6-Dimethylheptyl)oxy)-[1,1'-biphenyl]-4-carbonyl)oxy)-[1,1'-biphenyl]-4-yl 4'-(octyloxy)-[1,1'-biphenyl]-4-carboxylate, 4

Compound 4 was synthesized according to the procedure outlined for compound 1. Quantities: 3'-hydroxy-[1,1'-biphenyl]-4-yl-4'-(octyloxy)-[1,1'-biphenyl]-4-carboxylate 9c (132 mg, 0.267 mmol), (R)-4'-((6-methylheptan-2-yl)oxy)-[1,1'-biphenyl]-4-carboxylic acid 7a (107.6 mg, 0.33 mmol), 4-(dimethylamino)pyridine (DMAP) (41.6 mg, $0.34 \mathrm{mmol}$ ), and EDCI (66.4 mg, 0.35 mmol). The final product was a white solid ( $85 \mathrm{mg}, 40 \%) .{ }^{1} \mathrm{H} \mathrm{NMR}\left(\mathrm{CDCl}_{3}, 400 \mathrm{MHz}, \delta / \mathrm{ppm}\right): \delta$ 8.32 (dd, $4 \mathrm{H}, J=8.44 \mathrm{~Hz}, 1.68$ ), 7.70-7.76 (m, 6H, biphenyl), 7.65 (dt, 4H, $J=8.80 \mathrm{~Hz}, 3.04 \mathrm{~Hz}$, biphenyl), 7.55 (dt, 2H, $J=1.72 \mathrm{~Hz}, 8.64 \mathrm{~Hz}$, biphenyl), 7.51 (quin, 1H, biphenyl), 7.35 (dd, 2H, $J=6.40 \mathrm{~Hz}, 1.60 \mathrm{~Hz}$, biphenyl), $7.29(\mathrm{~m}, 1 \mathrm{H}$, biphenyl), 7.05 (dt, $4 \mathrm{H}, J=8.80 \mathrm{~Hz}, 3.3 \mathrm{~Hz}$, biphenyl), 4.03 - $4.12\left(\mathrm{~m}, 4 \mathrm{H}, 2 \mathrm{H}+2 \mathrm{H},-\mathrm{OCH}_{2}-\mathrm{C}_{7} \mathrm{H}_{15},-\mathrm{OCH}_{\underline{a}} \underline{\underline{H}} \underline{\underline{b}} \mathrm{CH}\left(\mathrm{CH}_{3}\right)\right), 1.82-1.95(\mathrm{~m}, 1 \mathrm{H}+2 \mathrm{H}$, $\left.-\mathrm{OCH}_{2} \underline{\mathrm{CH}}\left(\mathrm{CH}_{3}\right)\left(\mathrm{CH}_{2}\right)_{3} \mathrm{CH}_{\mathrm{a}}\left(\mathrm{CH}_{3}\right)_{3},-\mathrm{OCH}_{2} \underline{\mathrm{CH}}_{2}\left(\mathrm{CH}_{2}\right)_{5} \mathrm{CH}_{3}\right), 1.30-1.74(\mathrm{~m}, 17 \mathrm{H}), 0.91-1.01(\mathrm{~m}$, $\left.3 \mathrm{H}+9 \mathrm{H},-\mathrm{O}\left(\mathrm{CH}_{2}\right)_{7} \mathrm{CH}_{3},-\mathrm{OCH}_{2} \mathrm{CH}\left(\mathrm{CH}_{3}\right)\left(\mathrm{CH}_{2}\right)_{3}\left(\mathrm{CH}_{3}\right)_{2}\right) .{ }^{13} \mathrm{C} \mathrm{NMR}\left(\mathrm{CDCl}_{3}, 100 \mathrm{MHz}, \delta / \mathrm{ppm}\right)$ : 165.19, 159.62, 151.47, 150.77, 146.06, 142.09, 137.98, 130.80, 128.34, 126.63, 122.17, 115.01, $77.07,76.76,68.18,66.49,39.29,37.33,36.20,31.87,29.89,29.42,28.02,26.10,24.72,22.77$, 22.72, 22.67, 19.71, 14.18. Elemental analysis: calculated for $\mathrm{C}_{55} \mathrm{H}_{60} \mathrm{O}_{6}: \mathrm{C}, 80.85 ; \mathrm{H}, 7.40$; Found: C, 80.62; H, 7.32 .

\section{(R)-4'-((4'-((2,6-Dimethylheptyl)oxy)-[1,1'-biphenyl]-4-carbonyl)oxy)-[1,1'-biphenyl]-3-yl 3'-(octyloxy)-[1,1'-biphenyl]-4-carboxylate, 5}

Compound 5 was synthesized following the procedure described for 1 above. Quantities: 4'-(3hydroxyphenyl)phenyl-4-(4-\{[(2R)-2,6-dimethylheptyl $]$ oxy $\})-[1,1$ '-biphenyl]-4-carboxylate 9a (80 mg, $0.158 \mathrm{mmol}), 4-[4-($ octyloxy)phenyl]benzoic acid 7c (64 mg, $0.196 \mathrm{mmol})$, and DMAP 
(25.14 mg, $0.205 \mathrm{mmol})$, EDCI (13.80 mg, $0.072 \mathrm{mmol})$. The final product was a white solid (64 $\mathrm{mg}, 50 \%) .{ }^{1} \mathrm{H} \mathrm{NMR}\left(\mathrm{CDCl}_{3}, 400 \mathrm{MHz}, \delta / \mathrm{ppm}\right): \delta 8.32(\mathrm{dd}, 4 \mathrm{H}, J=8.44 \mathrm{~Hz}, 1.68), 7.70-7.76(\mathrm{~m}$, $6 \mathrm{H}$, biphenyl), 7.65 (dt, 4H, $J=8.80 \mathrm{~Hz}, 3.04 \mathrm{~Hz}$, biphenyl), $7.55(\mathrm{dt}, 2 \mathrm{H}, J=1.72 \mathrm{~Hz}, 8.64 \mathrm{~Hz}$, biphenyl), 7.51 (quin, $1 \mathrm{H}$ biphenyl), 7.35 (dd, 2H, $J=6.40 \mathrm{~Hz}, 1.60 \mathrm{~Hz}$, biphenyl), 7.29 (m, 1H, biphenyl), 7.05 (dt, $4 \mathrm{H}, J=8.80 \mathrm{~Hz}, 3.3 \mathrm{~Hz}$, biphenyl), 4.03-4.12 (m, 4H, $2 \mathrm{H}+2 \mathrm{H},-\mathrm{OCH}_{2}-\mathrm{C}_{7} \mathrm{H}_{15}$, $\left.-\underline{\mathrm{OCH}}_{\underline{a}} \underline{\mathrm{H}_{b}} \underline{\mathrm{CH}}\left(\mathrm{CH}_{3}\right)\right), \quad 1.82-1.95 \quad\left(\mathrm{~m}, \quad 1 \mathrm{H}+2 \mathrm{H}, \quad-\mathrm{OCH}_{2} \underline{\mathrm{CH}}\left(\mathrm{CH}_{3}\right)\left(\mathrm{CH}_{2}\right)_{3} \mathrm{CH}_{\mathrm{a}}\left(\mathrm{CH}_{3}\right)_{3}, \quad-\mathrm{OCH}_{2} \underline{\mathrm{CH}_{2}}\right.$ $\left.\left(\mathrm{CH}_{2}\right)_{5} \mathrm{CH}_{3}\right), 1.21-1.74\left(\mathrm{~m}, 17 \mathrm{H}, \mathrm{CH}_{2}\right), 0.91-1.04\left(3 \mathrm{H}+9 \mathrm{H},-\mathrm{O}\left(\mathrm{CH}_{2}\right)_{7} \mathrm{CH}_{3},-\mathrm{OCH}_{2} \mathrm{CH}\left(\mathrm{CH}_{3}\right)\left(\mathrm{CH}_{2}\right)_{3}\right.$ $\left.\left(\mathrm{CH}_{3}\right)_{2}\right) \cdot{ }^{13} \mathrm{C}$ NMR $\left(\mathrm{CDCl}_{3}, 100 \mathrm{MHz}, \delta / \mathrm{ppm}\right): 165.17,159.61,151.46,150.76,142.09,137.99$, 131.96, 130.79, 129.88, 128.34, 127.44, 127.40, 126.64, 124.64, 122.16, 120.69, 120.53, 115.01, $77.05,76.73,68.18,66.49,39.27,37.32,36.19,31.83,29.88,29.11,28.02,26.05,24.70,22.76$, 22.66, 19.70, 14.15. Elemental analysis: calculated for $\mathrm{C}_{55} \mathrm{H}_{60} \mathrm{O}_{6}: \mathrm{C}, 80.85$; H, 7.40; Found: $\mathrm{C}$, 80.67; H 7.22. 
NMR spectra - final compounds $\mathbf{1}$ - 5

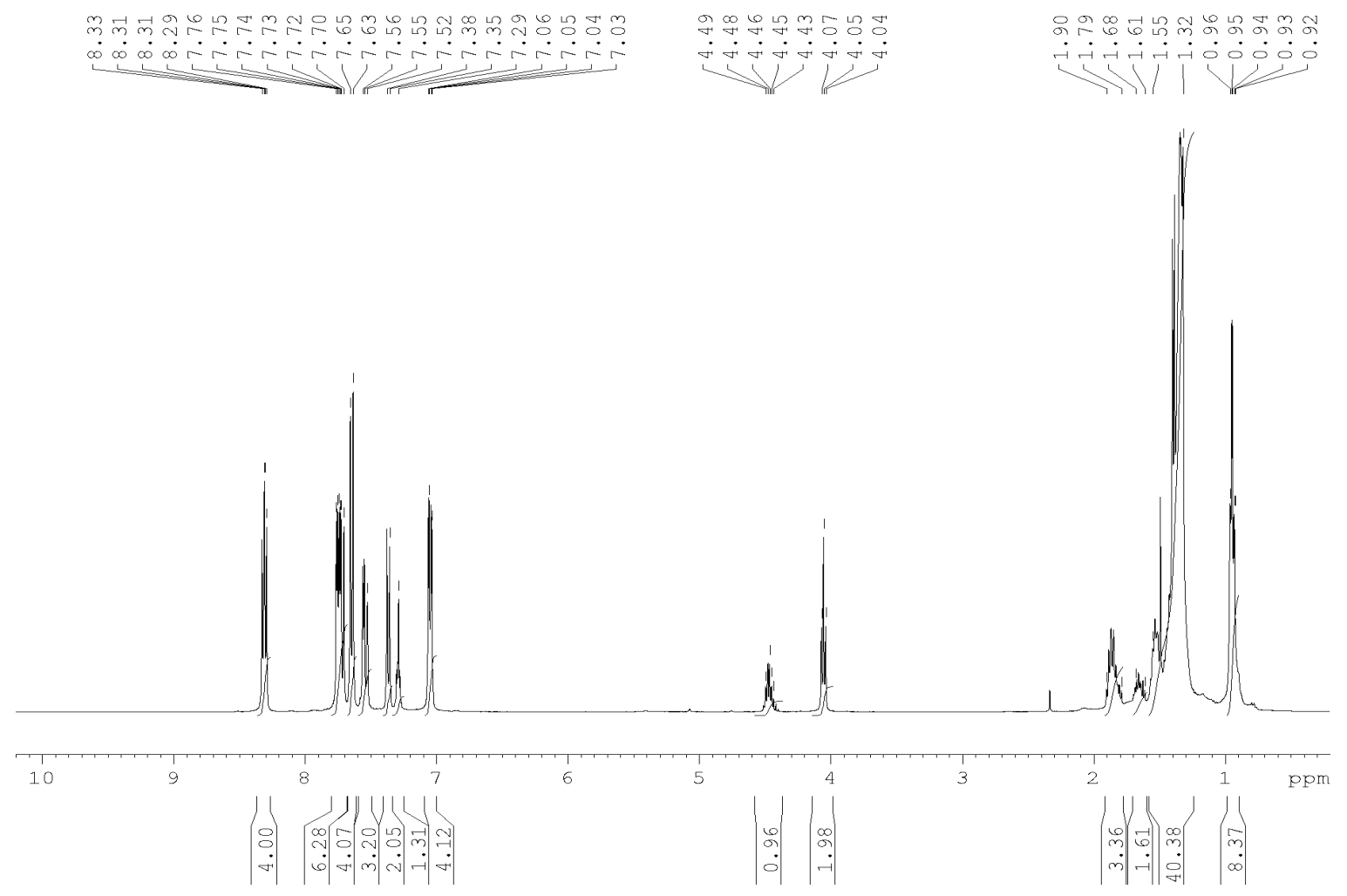

Figure S1. ${ }^{1} \mathrm{H}$ NMR spectrum of $\mathbf{1}$.

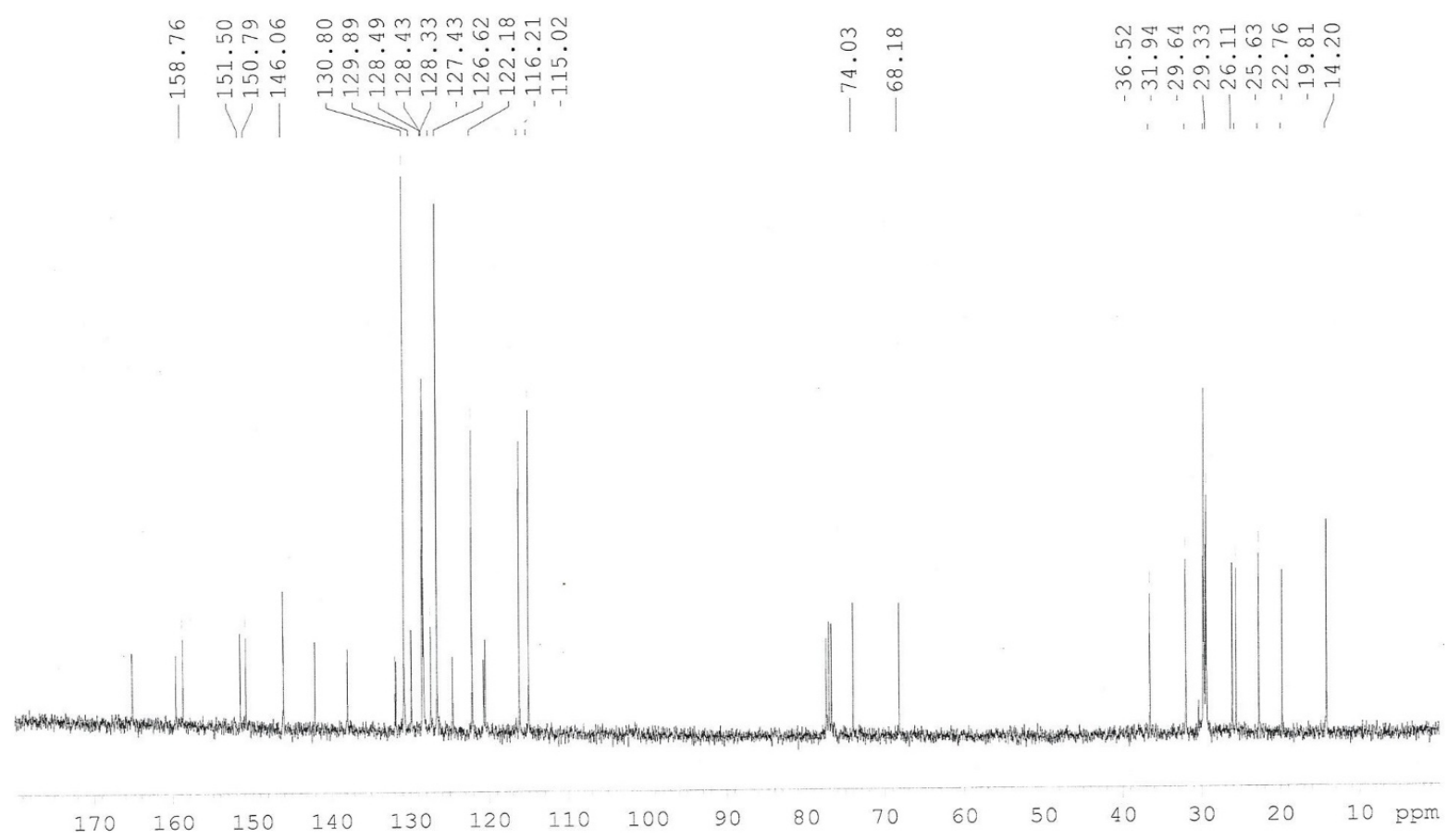

Figure S2. ${ }^{13} \mathrm{C}$ NMR spectrum of $\mathbf{1}$. 

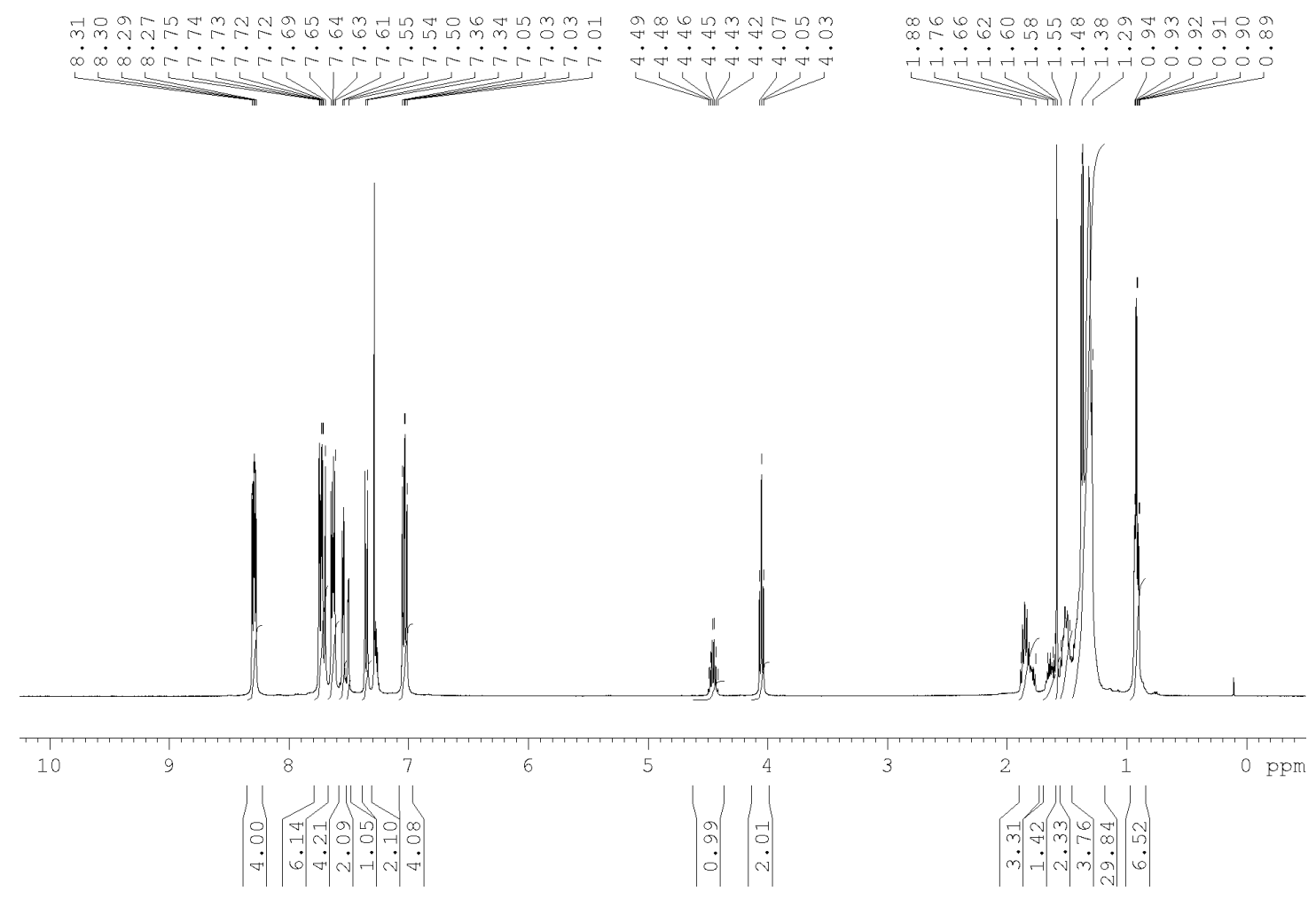

Figure S3. ${ }^{1} \mathrm{H}$ NMR spectrum of 2.

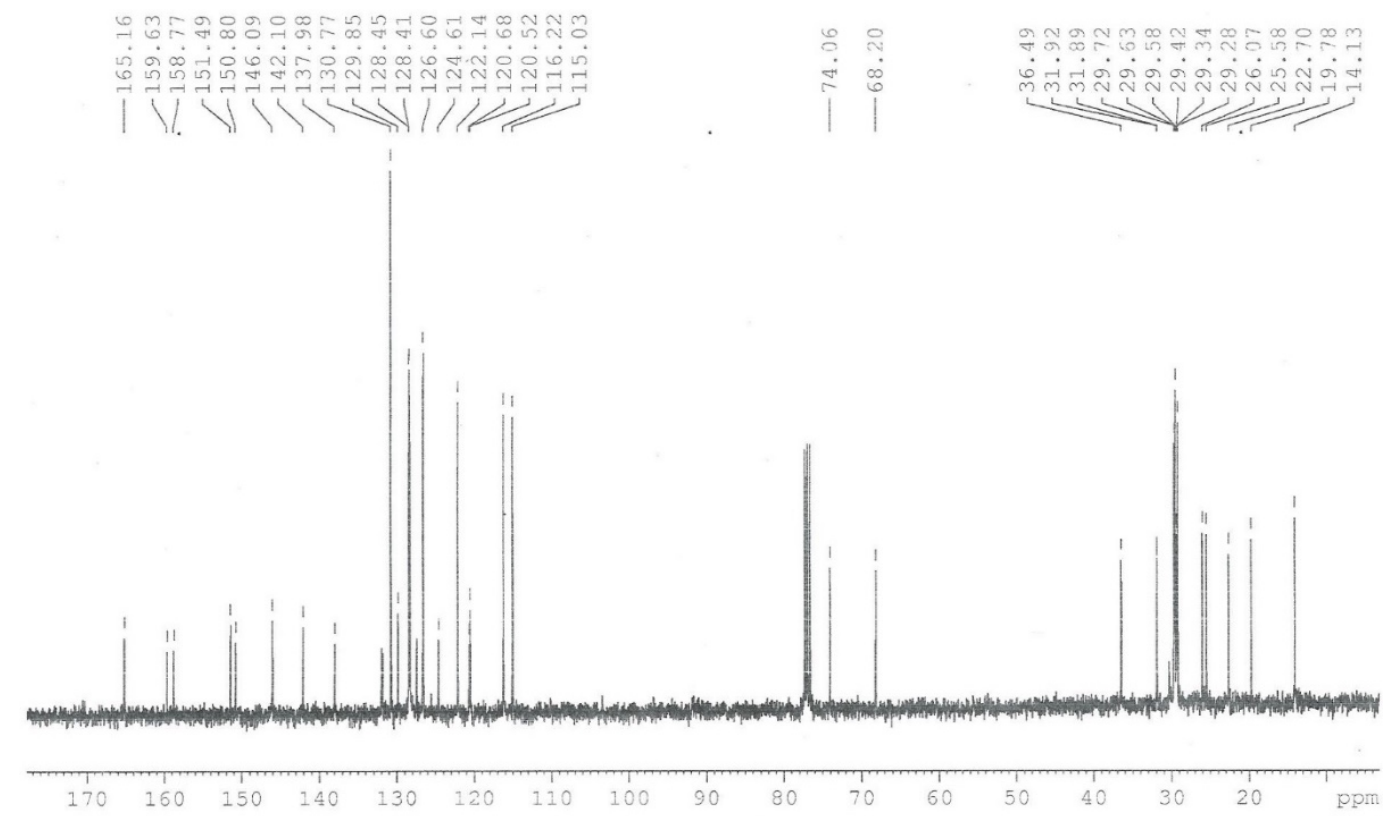

Figure S4. ${ }^{13} \mathrm{C}$ NMR spectrum of 2 . 


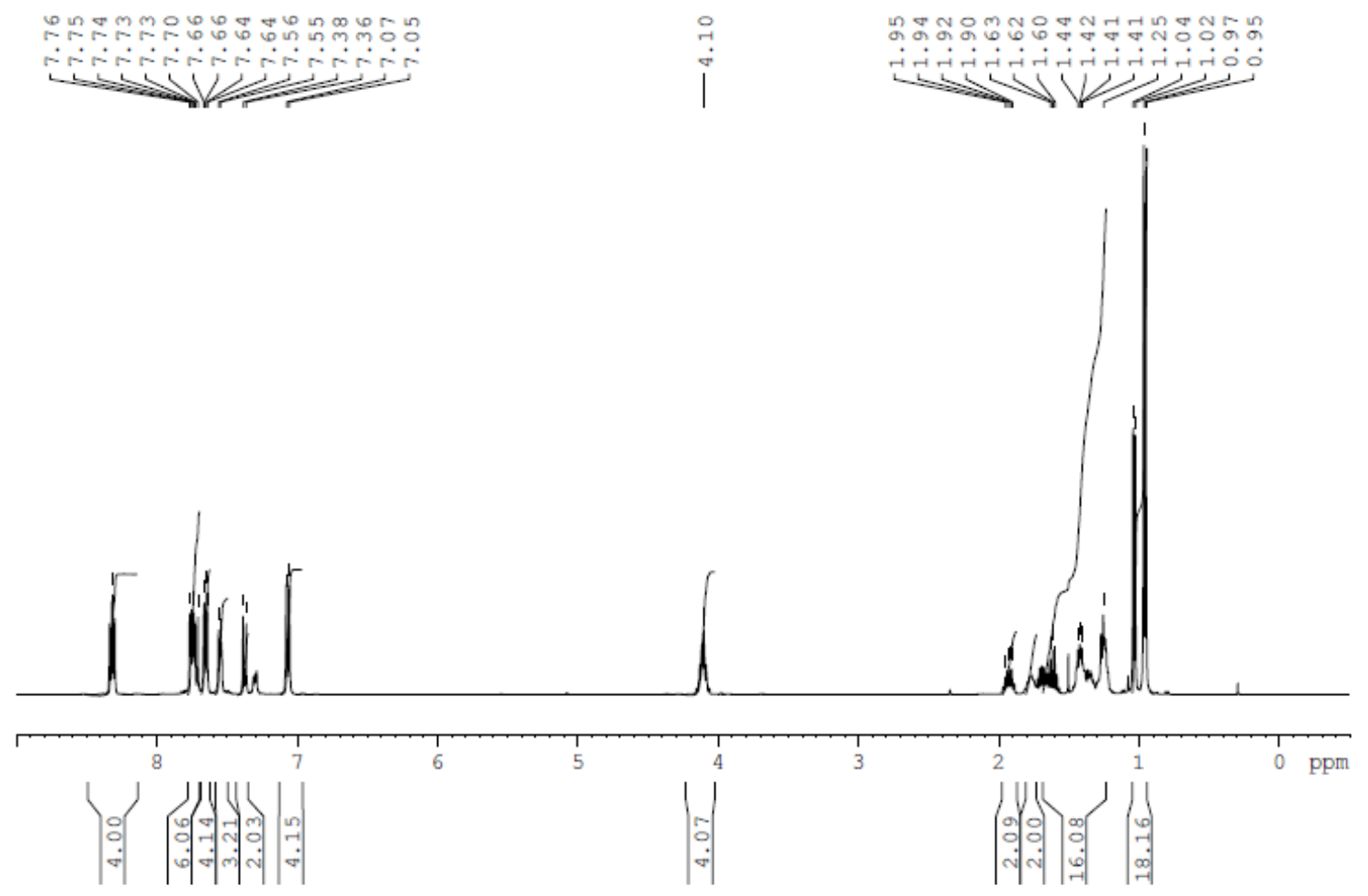

Figure S5. ${ }^{1} \mathrm{H}$ NMR spectrum of 3 .

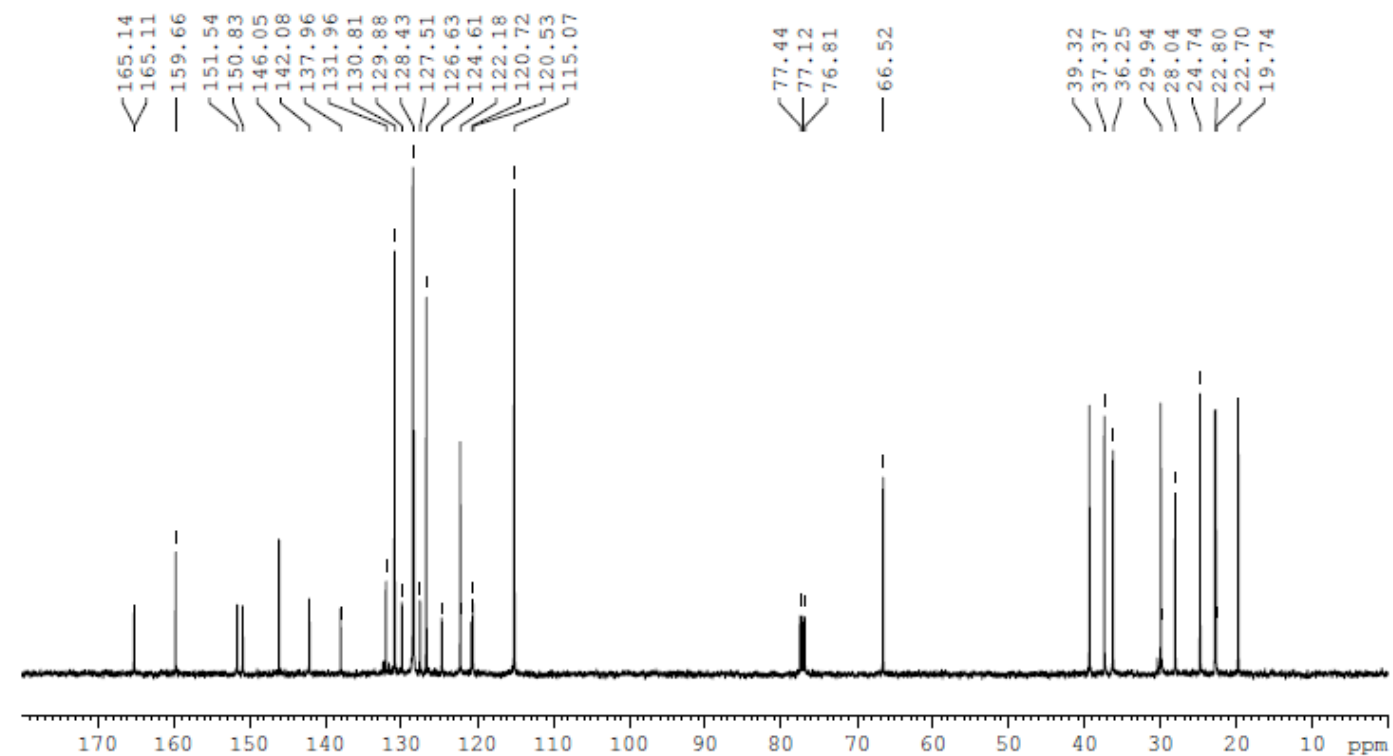

Figure S6. ${ }^{13} \mathrm{C}$ NMR spectrum of 3 . 


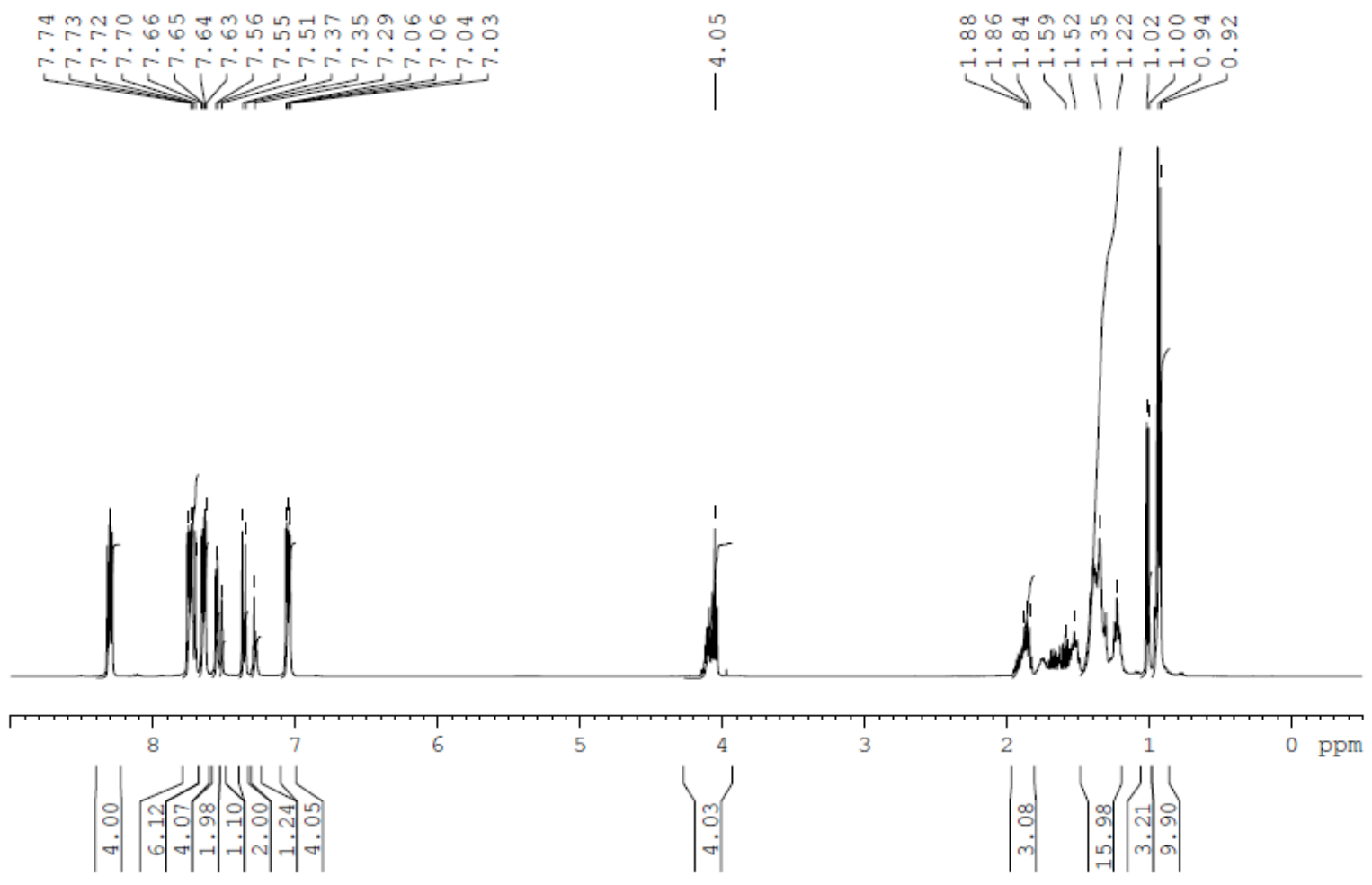

Figure S7. ${ }^{1} \mathrm{H}$ NMR spectrum of 4 .

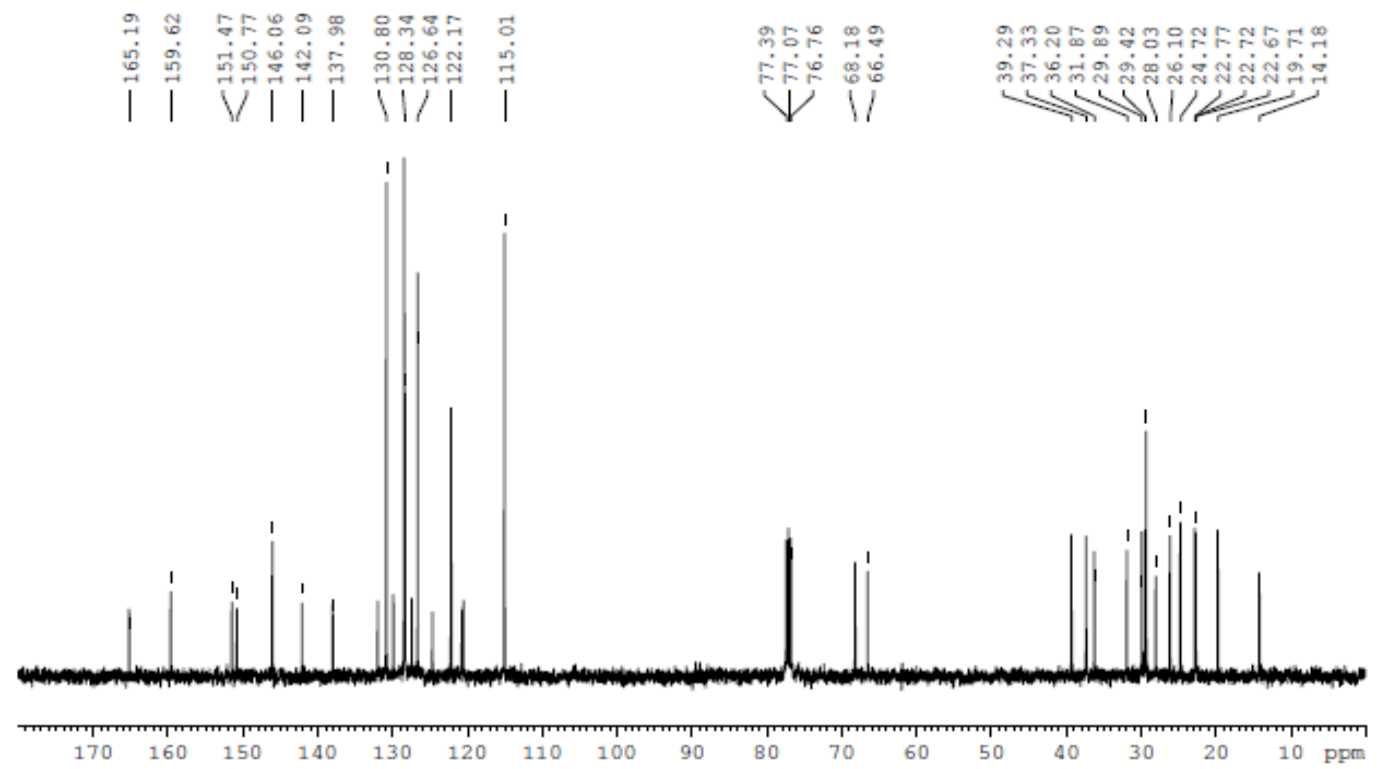

Figure S8. ${ }^{13} \mathrm{C}$ NMR spectrum of 4 . 


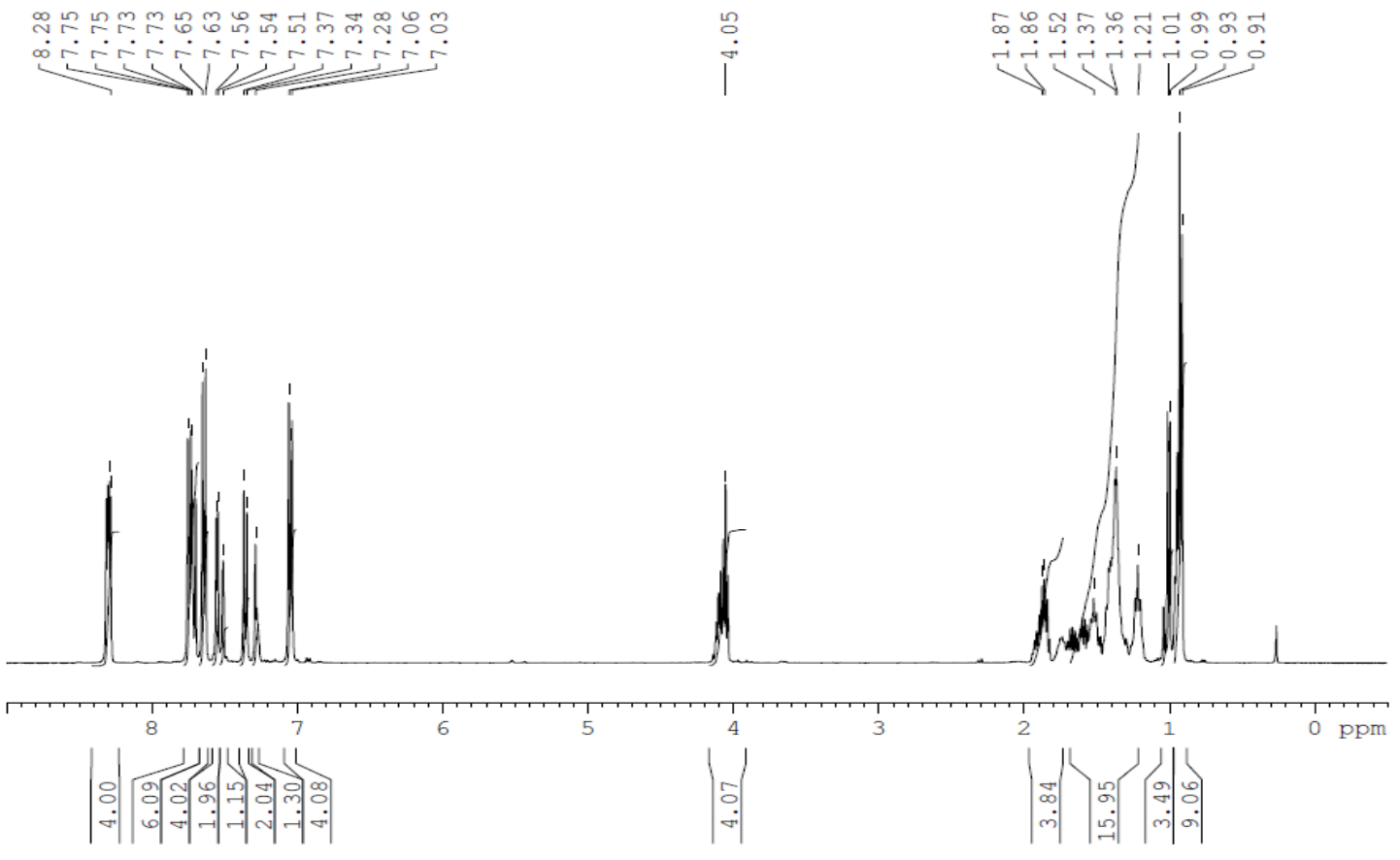

Figure S9. ${ }^{1} \mathrm{H}$ NMR spectrum of 5 .

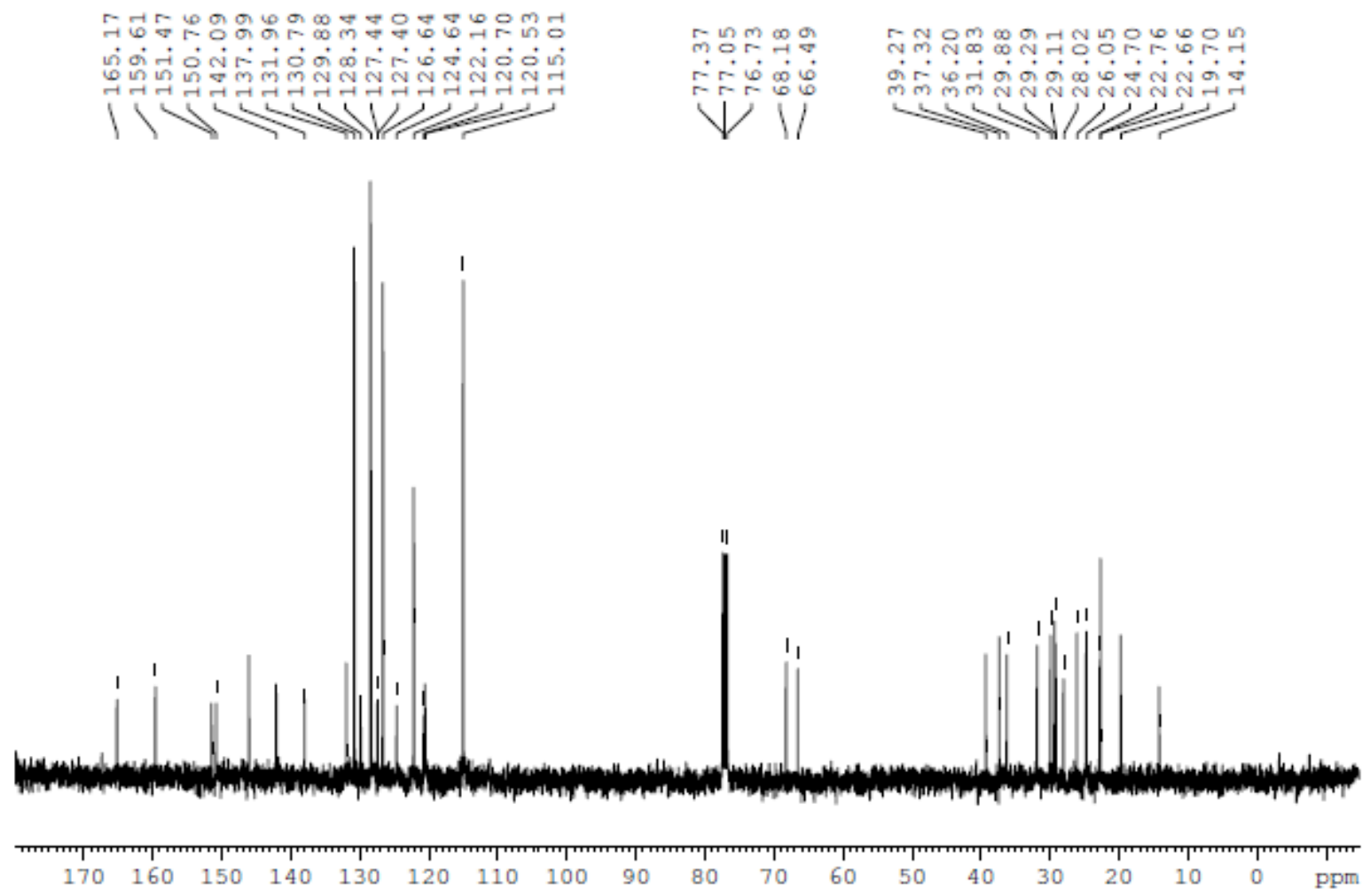

Figure S10. ${ }^{13} \mathrm{C}$ NMR spectrum of 5 . 


\section{Section S2. Additional Data for Compound 1}

\section{DSC data}

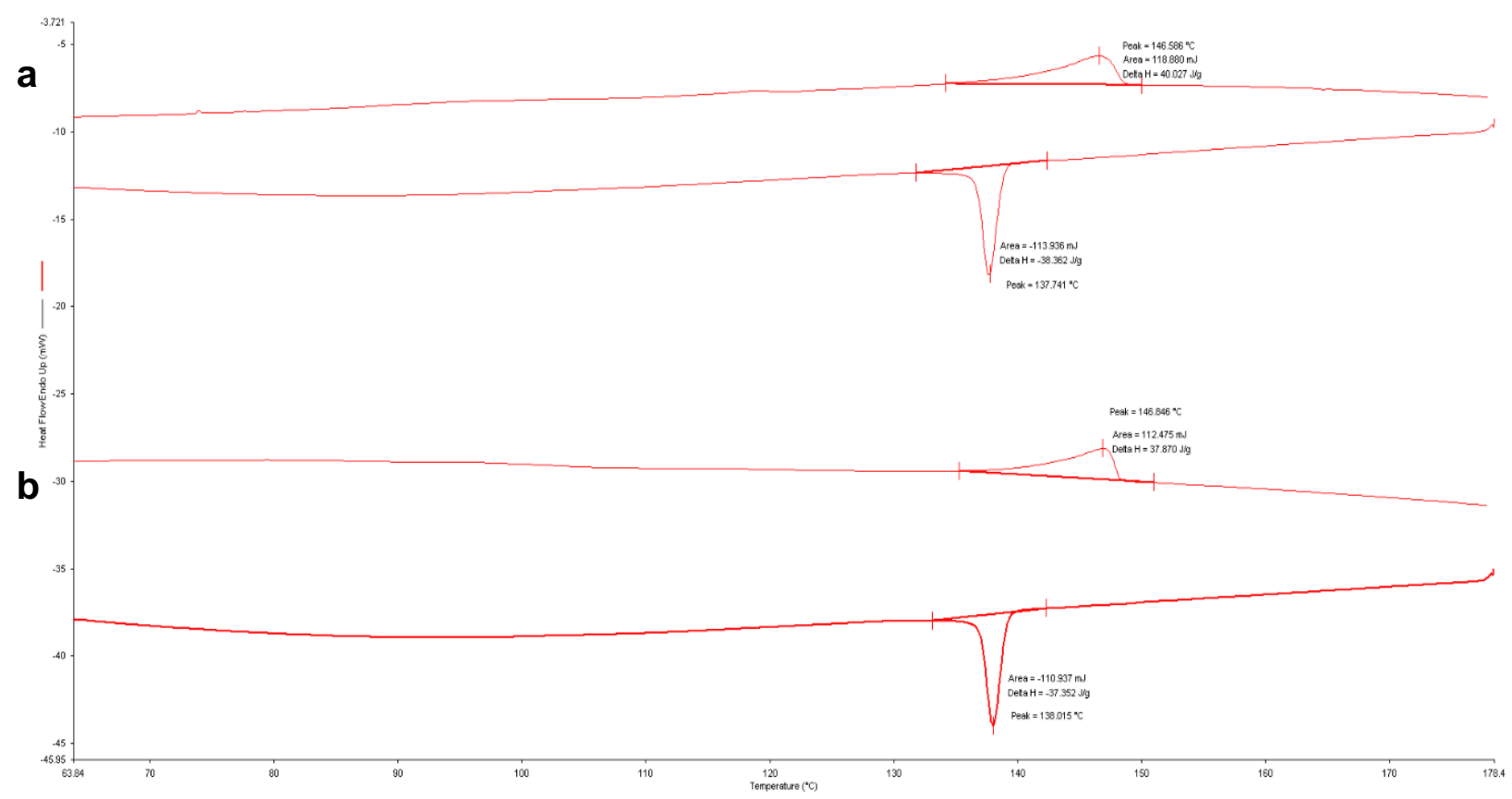

Figure S11. DSC thermograms of compound 1 at a heating/cooling rate of $5{ }^{\circ} \mathrm{C} \min ^{-1}$ : (a) $1^{\text {st }}$ heating and cooling runs and (b) $2^{\text {nd }}$ heating and cooling runs.

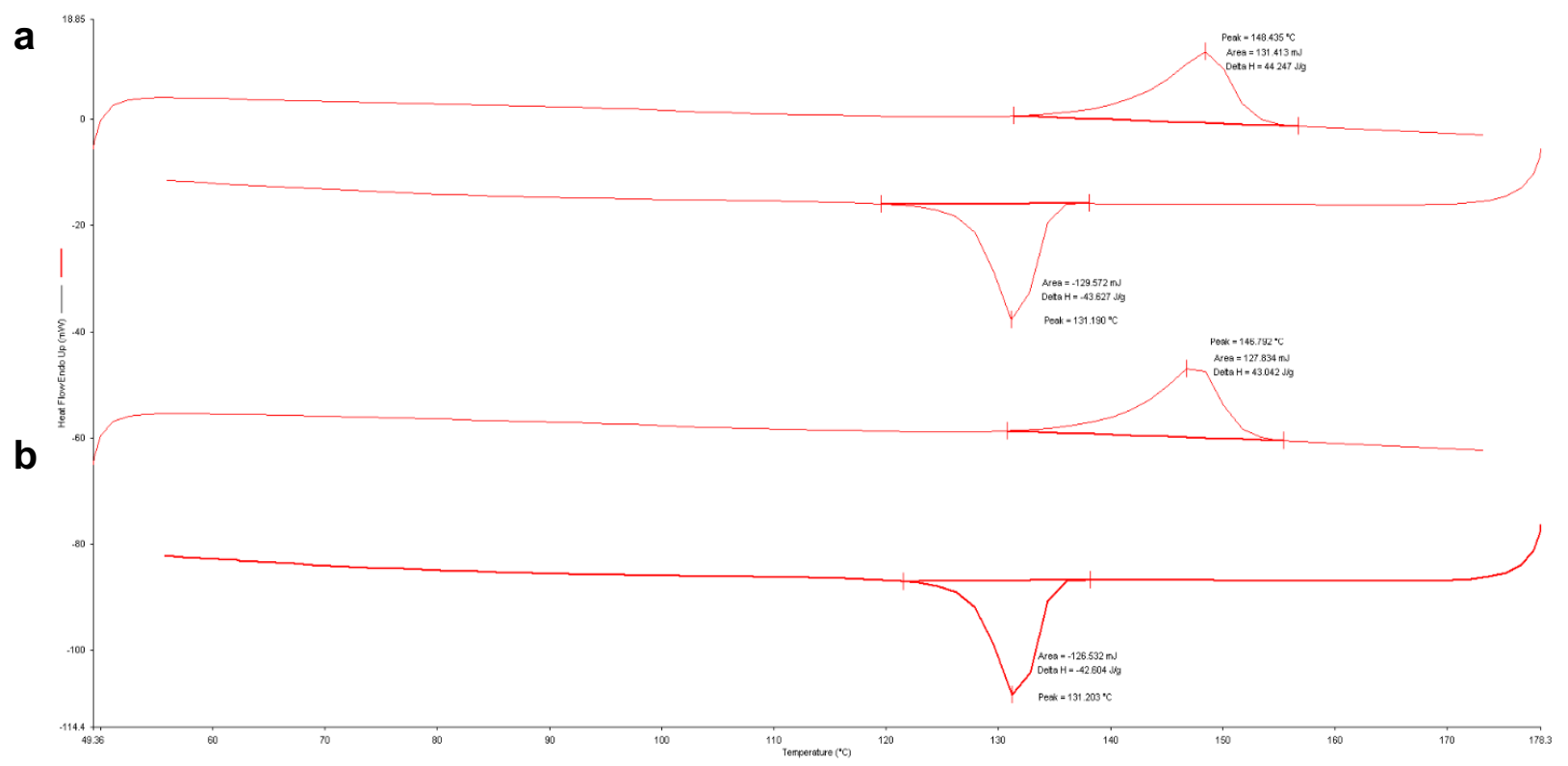

Figure S12. DSC thermograms of compound 1 at a heating/cooling rate of $50{ }^{\circ} \mathrm{C} \mathrm{min}^{-1}$ : (a) $1^{\text {st }}$ heating and cooling runs and (b) $2^{\text {nd }}$ heating and cooling runs. 
Table S1. DSC data for compound 1 at both heating/cooling rates.

\begin{tabular}{lccc}
\hline $\begin{array}{l}\text { Rate } \\
{\left[{ }^{\circ} \mathrm{min}^{-1}\right]}\end{array}$ & $\begin{array}{l}\text { Run } \\
\text { on Heating }{ }^{\circ} \mathrm{C}[\mathrm{kJ} / \mathrm{mol}]\end{array}$ & $\begin{array}{l}\text { Transition Temperature [Enthalpy] } \\
\text { on Cooling }{ }^{\circ} \mathrm{C}[\mathrm{kJ} / \mathrm{mol}]\end{array}$ \\
\hline 5 & $1^{\text {st }}$ & $146.6[34.3]$ & $137.7[-33.0]$ \\
& $2^{\text {nd }}$ & $147.1[32.5]$ & $138.0[-32.1]$ \\
50 & $1^{\text {st }}$ & $148.4[38.1]$ & $131.2[-37.5]$ \\
& $2^{\text {nd }}$ & $146.8[36.9]$ & $131.2[-36.6]$ \\
\hline
\end{tabular}




\section{Additional SEM and TEM images}
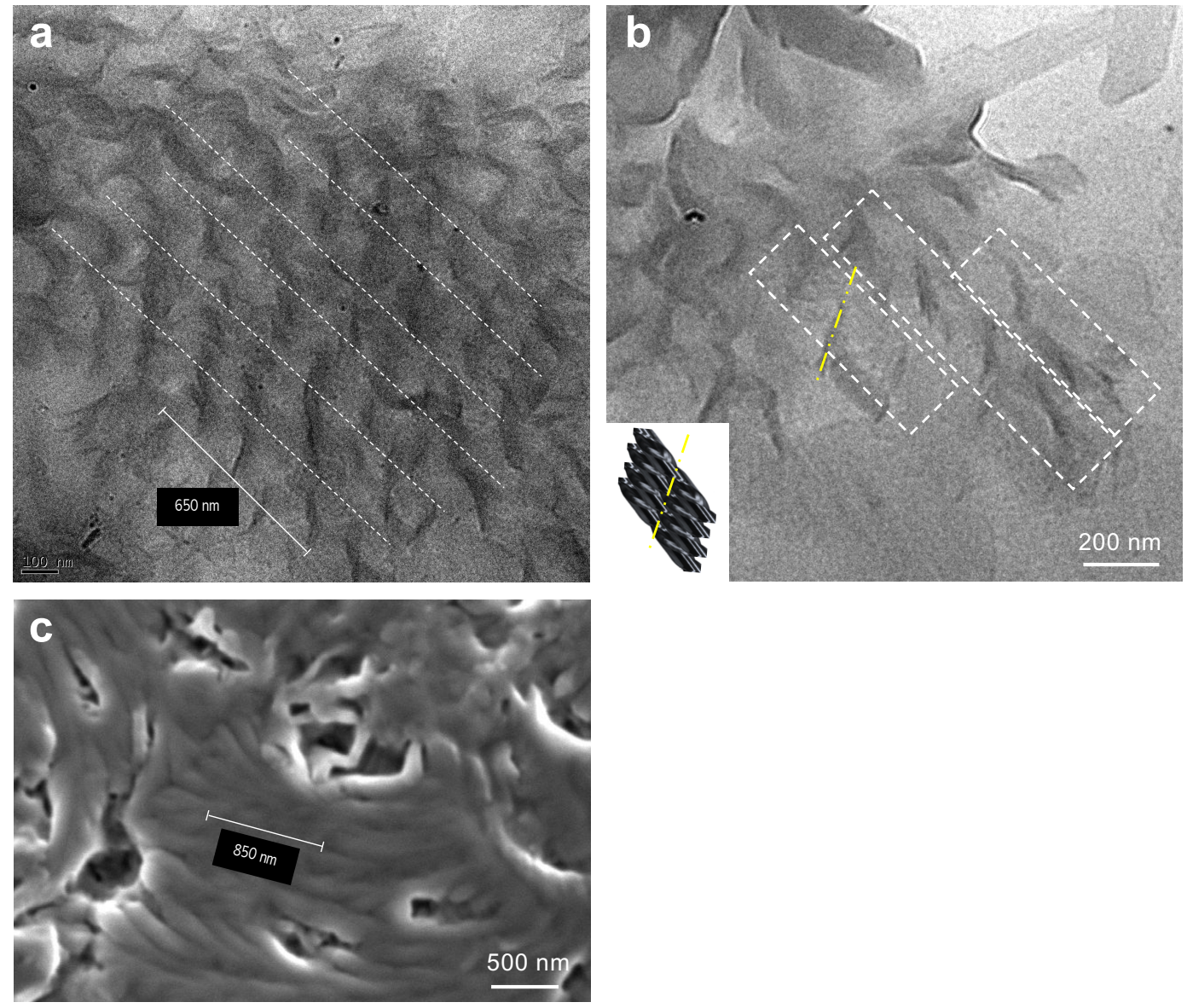

Figure S13. (a) TEM image of 1 taken after fast cooling from the isotropic liquid phase to room temperature; scale bar $=100 \mathrm{~nm}$. This image shows fully developed and fused parallel arrays of what appears to be a layer of $\mathrm{H} \mu \mathrm{Fs}$ likely running parallel to the substrate. The filaments' pitch can be determined by measuring the distance between the dark ridges of individual $\mathrm{H} \mu \mathrm{F}$ in this side-by-side arrangement of the $\mathrm{H} \mu \mathrm{Fs}$ (highlighted by the white dashed lines). Similar arrangements for HNFs have previously been described by Lavrentovich and Jákli. ${ }^{5}$ (b) TEM image of $\mathbf{1}$ taken after fast cooling from the isotropic liquid phase to room temperature showing more isolated and much better visible $\mathrm{H} \mu \mathrm{Fs}$ corroborating the analysis in Figure S13a and Figure 5h in the main article; inset shows graphic of parallel array of left-handed $\mathrm{H} \mu \mathrm{Fs}$ and expected contrast (highlighted by yellow line). (c) SEM image of 1 taken after fast cooling from the isotropic liquid phase to room temperature (with gold evaporation) showing a related domain of fused $\mathrm{H} \mu \mathrm{Fs}$. Overall, SEM and TEM images reveal helical pitch, $p$, values ranging from $p=650-850 \mathrm{~nm}$. 


\section{Additional XRD data}

a

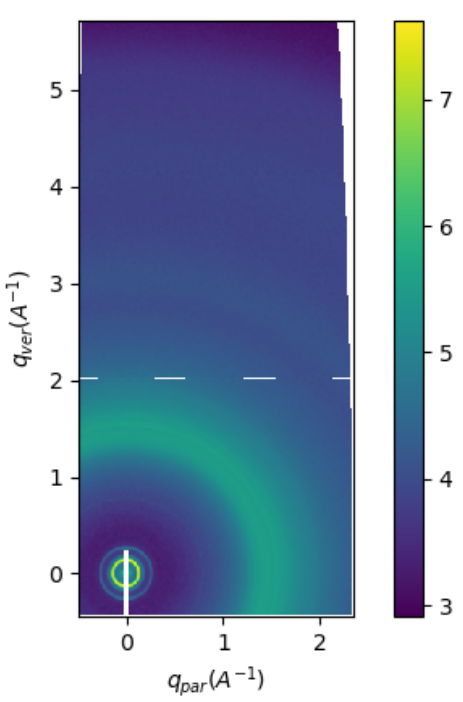

C

\begin{tabular}{|l|c|c|c|c|}
\hline$q / \AA^{-1}$ & & rel. intensity & \multicolumn{2}{|c|}{ index } \\
\hline 0.135 & $q_{1}^{a}$ & $8.79 \mathrm{E}+09$ & 001 & \\
\hline 0.164 & $q_{1}^{b}$ & & & 001 \\
\hline 0.187 & $q_{2}^{b}$ & & & 100 \\
\hline 0.201 & $q_{2}^{a}$ & $1.25 \mathrm{E}+08$ & 100 & \\
\hline 0.223 & $q_{3}^{b}$ & & & 101 \\
\hline 0.241 & $q_{3}^{a}$ & $1.17 \mathrm{E}+08$ & 101 & \\
\hline 0.270 & $q_{4}^{a}$ & $2.85 \mathrm{E}+08$ & 200 & \\
\hline 0.271 & $q_{4}^{b}$ & & & 101 \\
\hline 0.307 & $q_{5}^{b}$ & & & 010 \\
\hline 0.328 & $q_{6}^{b}$ & & & 002 \\
\hline 0.391 & $q_{5}^{a}$ & $7.97 \mathrm{E}+07$ & 004 & \\
\hline 0.382 & $q_{7}^{b}$ & & & 201 \\
\hline 0.783 & $q_{6}^{a}$ & $1.35 \mathrm{E}+08$ & 305 & \\
\hline 0.806 & $q_{8}^{b}$ & & & 201 \\
\hline
\end{tabular}

b

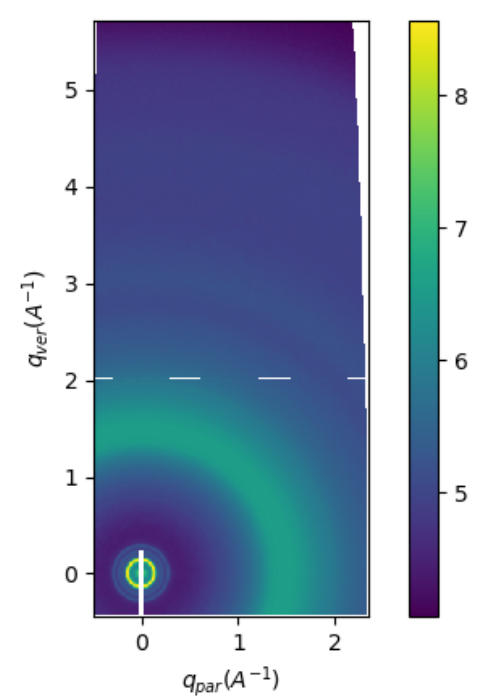

d $q / \AA^{-1} \quad$ rel. intensity index \begin{tabular}{|l|l|l|l|}
\hline 0.1415 & $q_{1}^{a}$ & $2.20 \mathrm{E}+10$ & 001 \\
\hline
\end{tabular} \begin{tabular}{l|l|l|l|}
0.226 & $q_{2}^{a}$ & $4.92 \mathrm{E}+08$ & 100
\end{tabular} \begin{tabular}{|l|l|l|l|}
0.2604 & $q_{3}^{a}$ & $3.52 \mathrm{E}+08$ & 101 \\
\hline
\end{tabular} \begin{tabular}{l|l|l|l|}
0.284 & $q_{4}^{a}$ & $5.42 \mathrm{E}+08$ & 002 \\
\hline
\end{tabular} \begin{tabular}{l|l|l|l|}
0.8059 & $q_{5}^{a}$ & $3.92 \mathrm{E}+08$ & 303 \\
\hline
\end{tabular}

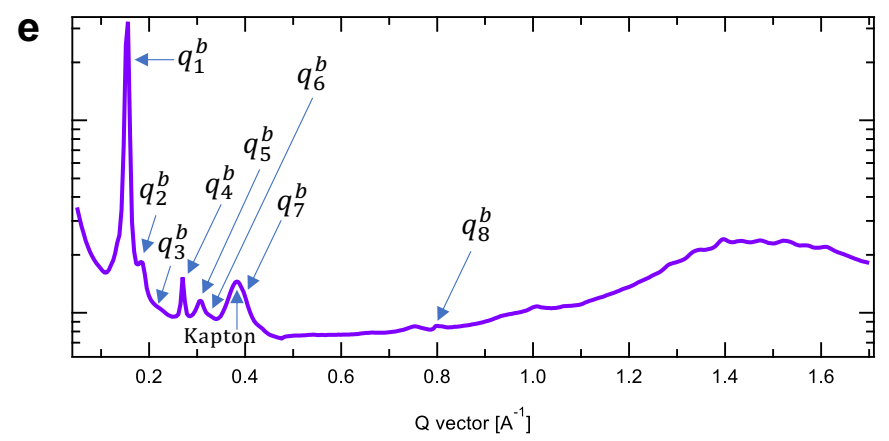

Figure S14. 2D X-ray diffraction (XRD) pattern obtained for compound 1 upon: (a) slow cooling and (b) fast cooling. Momentum transfer $(q)$, Miller indices, and relative intensities for compound 1 upon: (c) slow cooling (BNL and ALS datasets) and (d) fast cooling - BNL dataset. (e) Complementary ALS beamline data for compound $\mathbf{1}$ taken upon slow cooling from the isotropic liquid phase (used to support the $\mathrm{B} 1 \mathrm{Col}_{\mathrm{ob}}$ phase assignment and $p 2$ space group/lattice determination). 


\section{Section S3. Additional Data for Compound 2}

\section{DSC data}

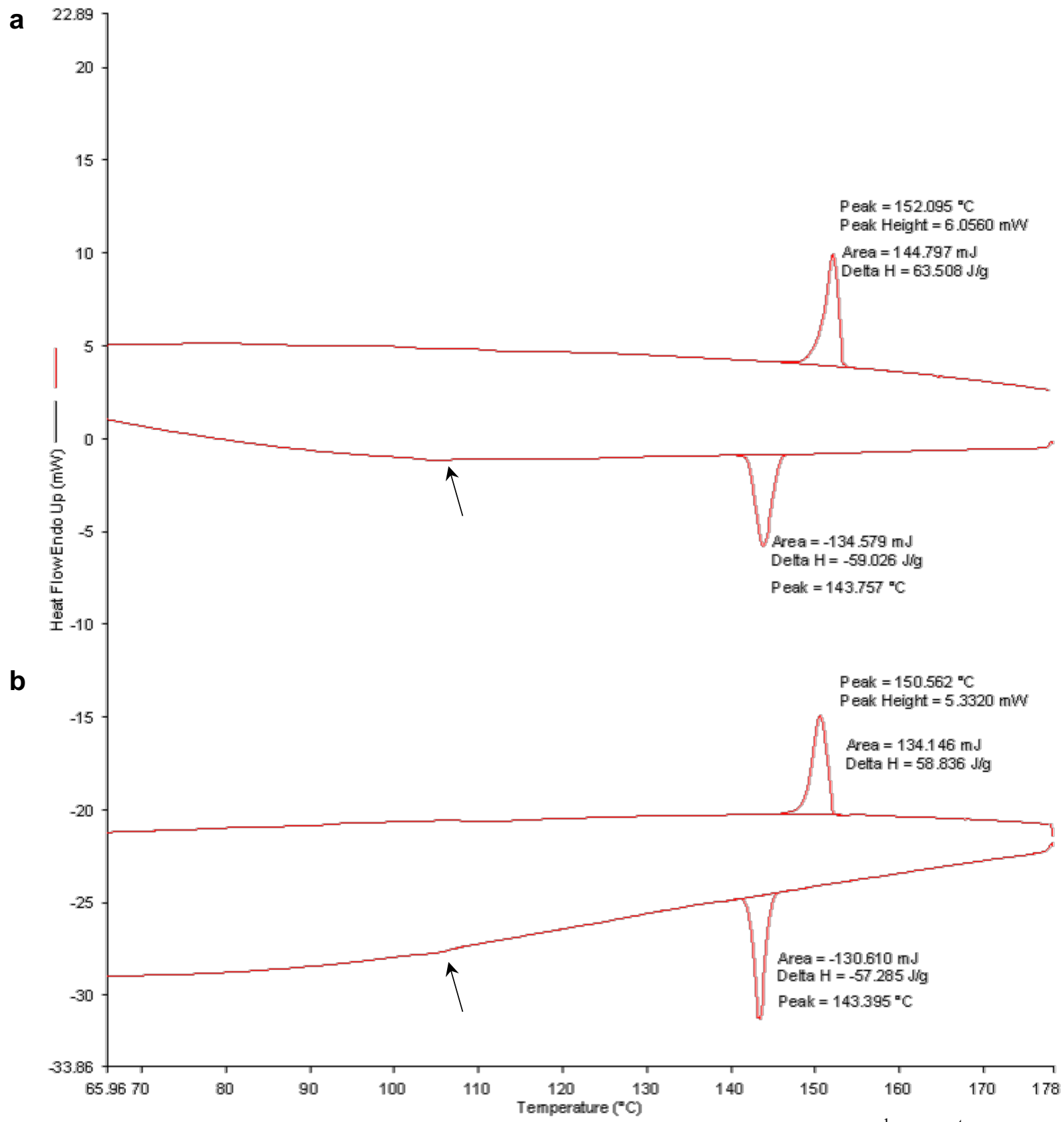

Figure S15. DSC thermograms of compound 2 at a heating/cooling rate of $5{ }^{\circ} \mathrm{C} \min ^{-1}$ : (a) $1^{\text {st }}$ heating and cooling runs and (b) $2^{\text {nd }}$ heating and cooling runs. Glass transitions, typically found for B4 materials, are barely visible in the cooling cycles; see arrows. 


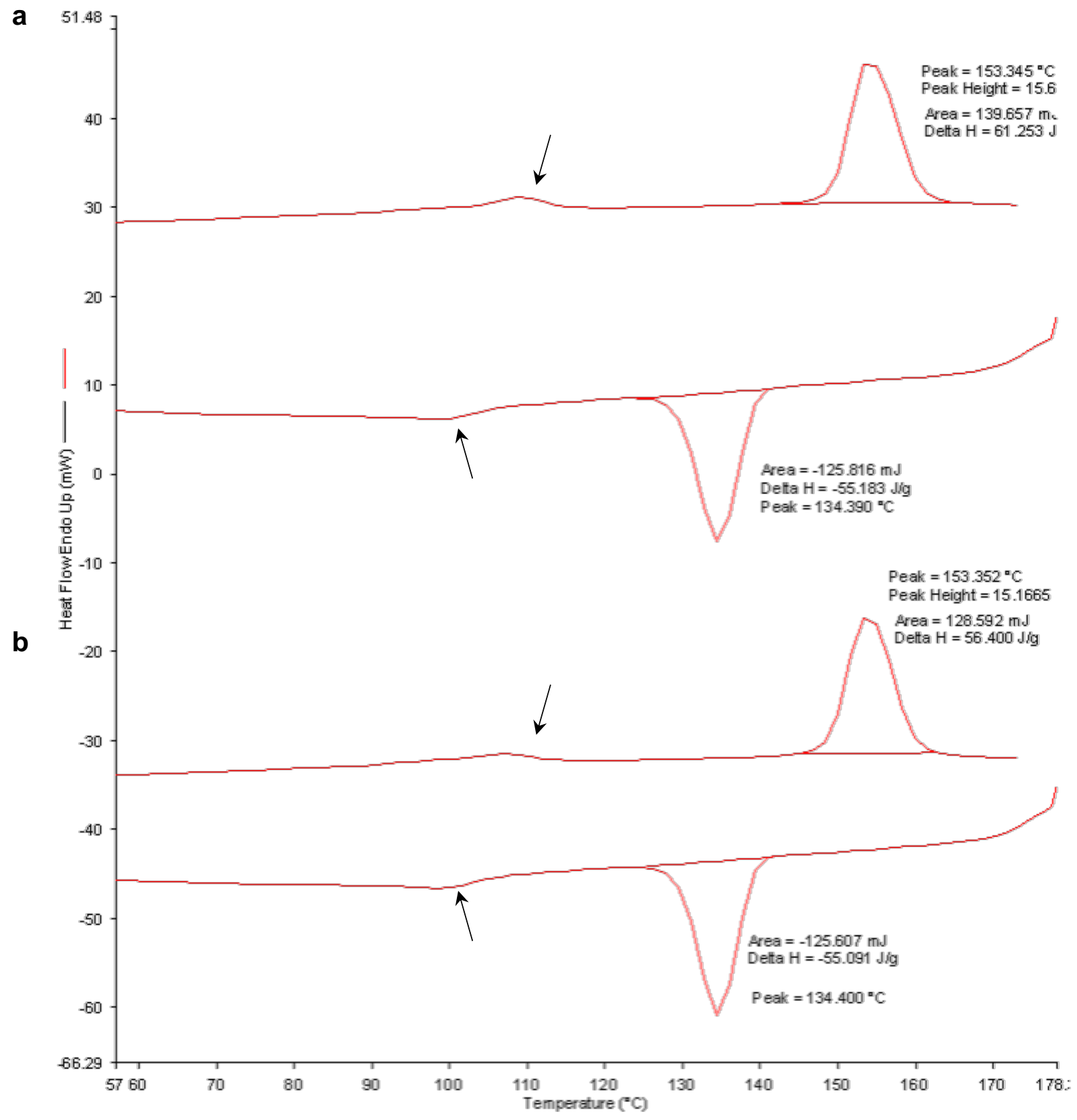

Figure S16. DSC thermograms of compound 2 at a heating/cooling rate of $50{ }^{\circ} \mathrm{C} \min ^{-1}$ : (a) $1^{\text {st }}$ heating and cooling runs and (b) $2^{\text {nd }}$ heating and cooling runs. Glass transitions are here clearly visible in each heating/cooling cycle (see arrows). 
Table S2. DSC data for compound 2 at both heating/cooling rates.

\begin{tabular}{lccc}
\hline $\begin{array}{l}\text { Rate } \\
{\left[{ }^{\circ} \mathrm{min}^{-1}\right]}\end{array}$ & Run & $\begin{array}{l}\text { Transition Temperature [Enthalpy] } \\
\text { on Heating }{ }^{\circ} \mathrm{C}[\mathrm{kJ} / \mathrm{mol}]\end{array}$ & $\begin{array}{l}\text { Transition Temperature [Enthalpy] } \\
\text { on Cooling }{ }^{\circ} \mathrm{C}[\mathrm{kJ} / \mathrm{mol}]\end{array}$ \\
\hline 5 & $1^{\text {st }}$ & $152.1[54.6]$ & $143.8[-50.7]$ \\
& $2^{\text {nd }}$ & $150.6[50.5]$ & $143.4[-49.2]$ \\
\hline 50 & $1^{\text {st }}$ & $153.3[52.6]$ & $134.4[-47.4]$ \\
& $2^{\text {nd }}$ & $153.4[48.5]$ & $134.4[-47.3]$ \\
\hline
\end{tabular}

\section{Additional SEM and TEM images}
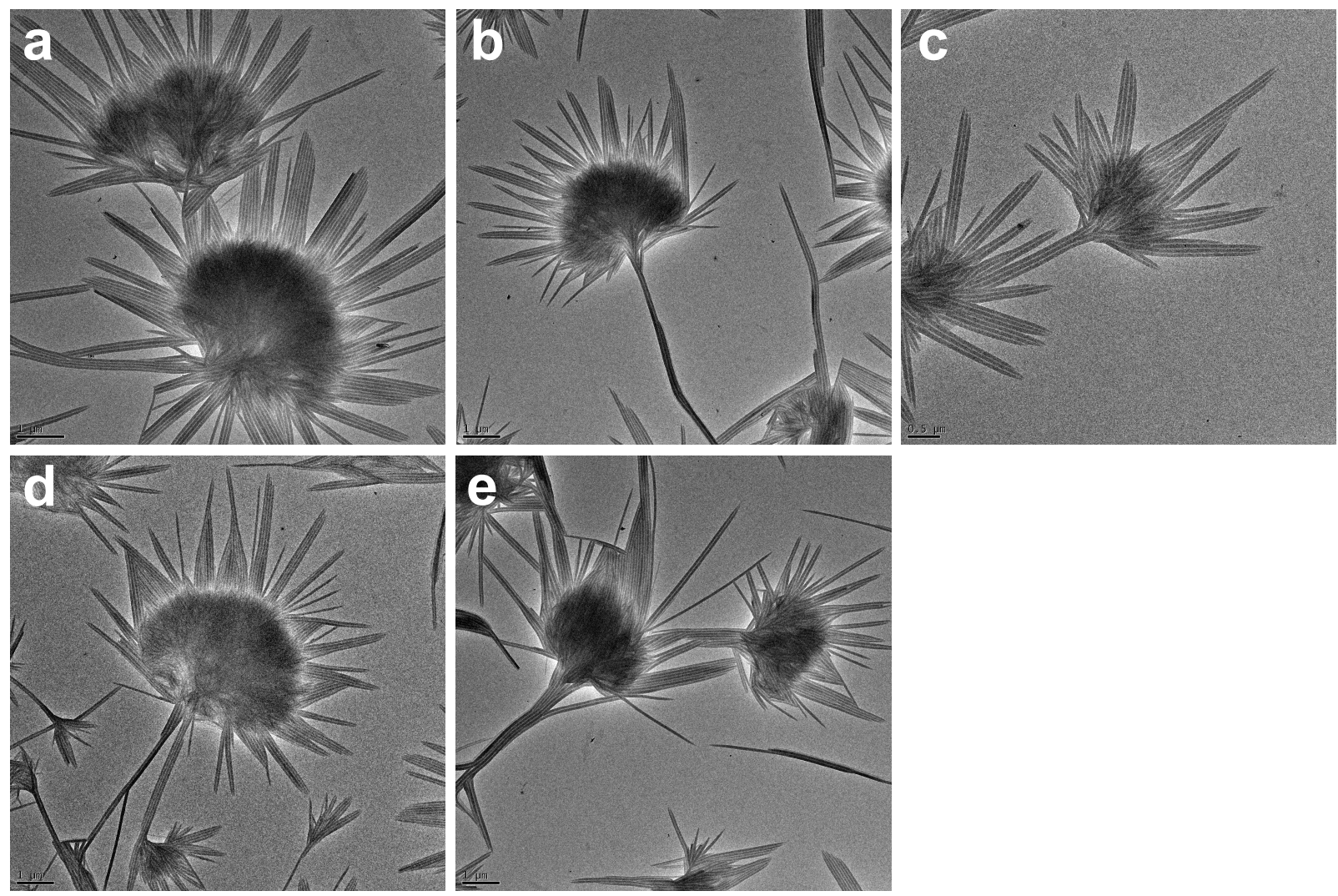

Figure S17. $(a-e)$ TEM images of $\mathbf{2}$ taken after slow cooling from the isotropic liquid phase to room temperature showing the analogy to the thistle flowers shown in the photographs; scale bars: $(a, b, d$, and e) $1 \mu \mathrm{m}$ and (c) $0.5 \mu \mathrm{m}$. 

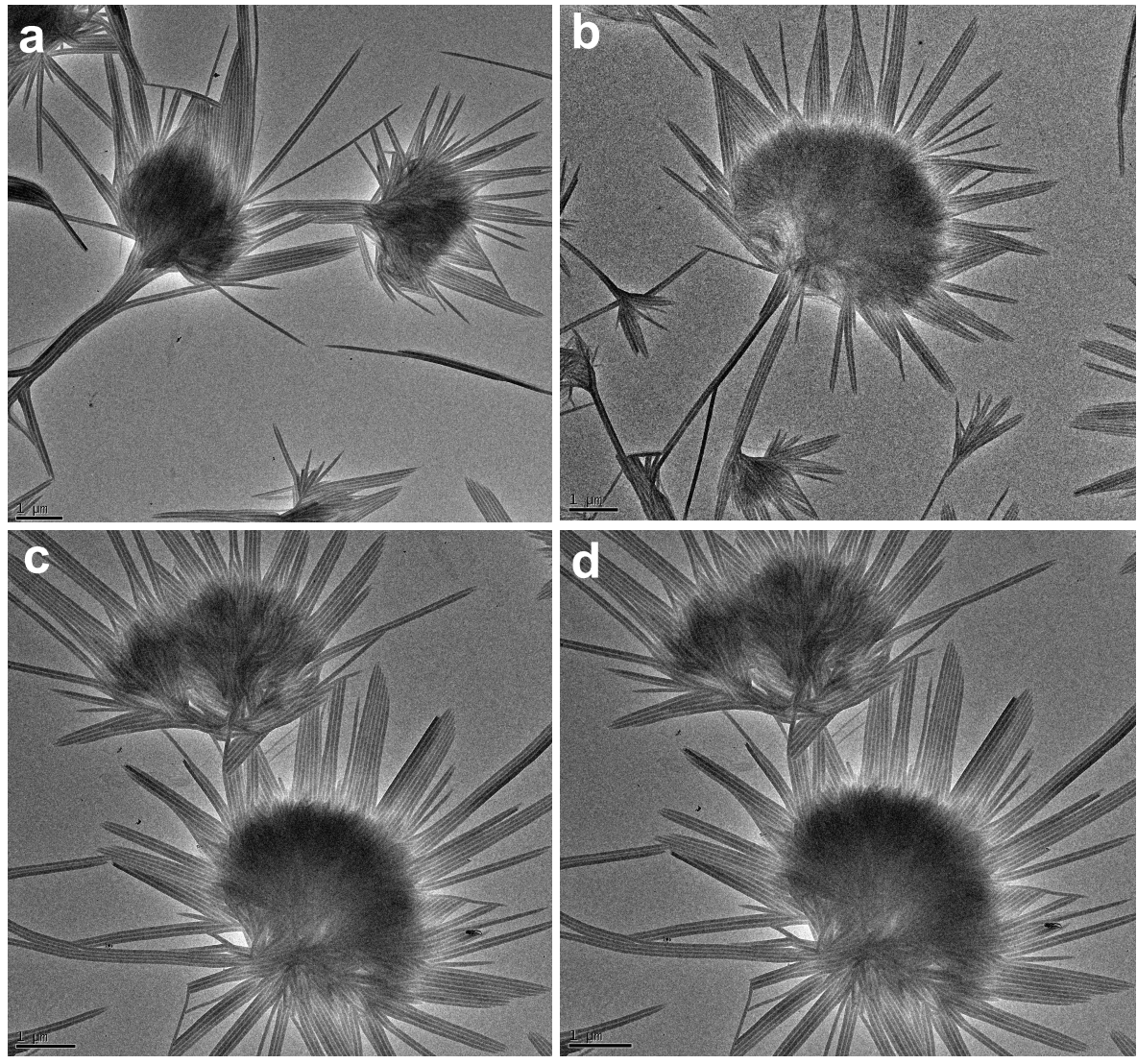

Figure S18. $(\mathrm{a}-\mathrm{d})$ TEM images of $\mathbf{2}$ taken after slow cooling from the isotropic liquid phase to room temperature showing the analogy to the thistle flowers shown in the photographs; scale bars: $1 \mu \mathrm{m}$.
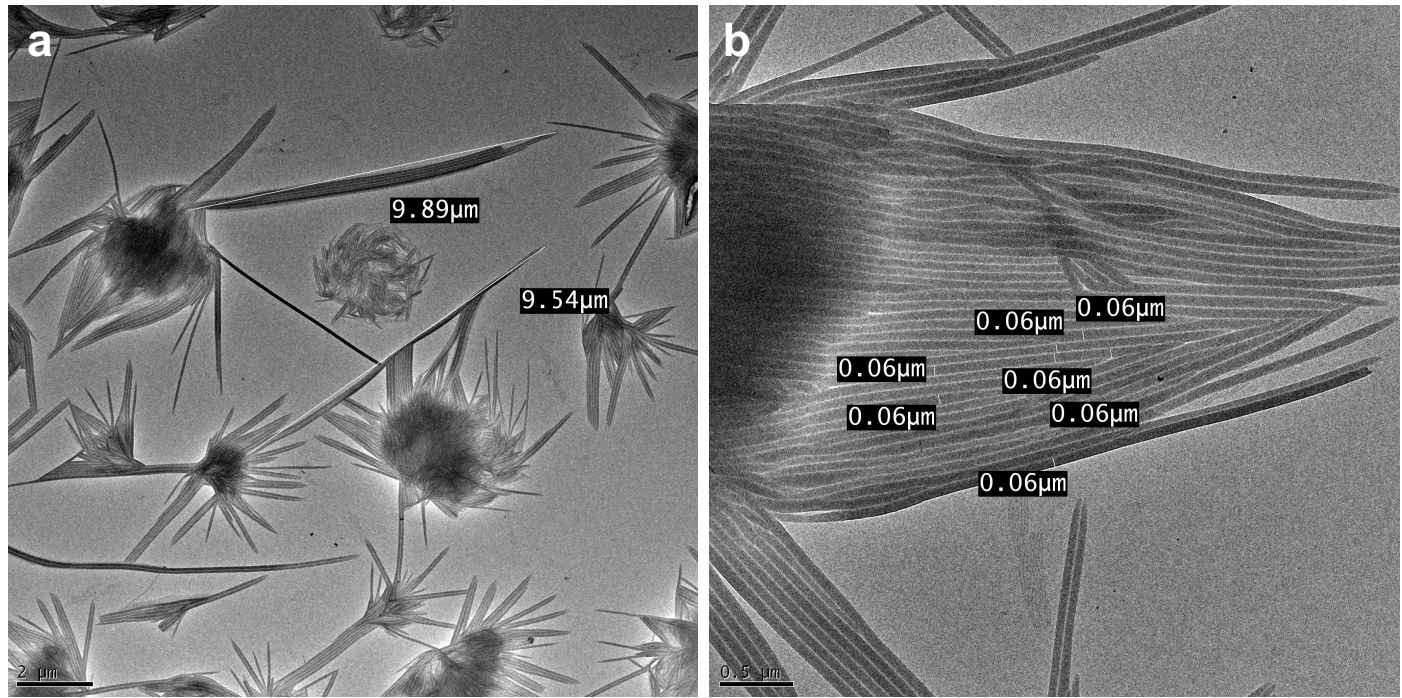

Figure S19. TEM images of $\mathbf{2}$ taken after slow cooling from the isotropic liquid phase to room temperature showing: (a) the length of these nanocylinders of several $\mu \mathrm{m}$ and (b) the constant diameter of $\sim 60 \mathrm{~nm}$; scale bars: (a) $2 \mu \mathrm{m}$ and (b) $0.5 \mu \mathrm{m}$. 

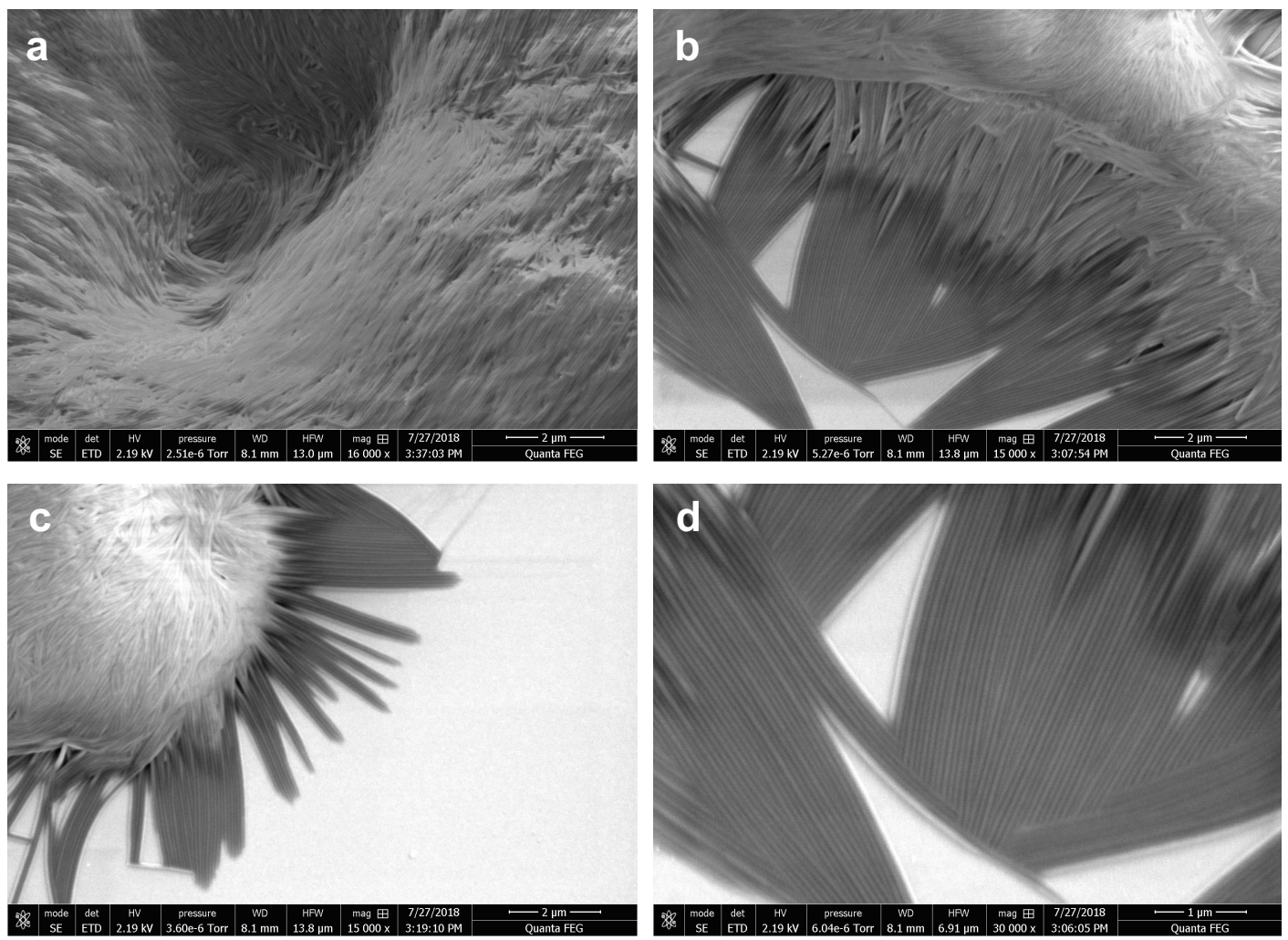

Figure S20. $(\mathrm{a}-\mathrm{d})$ Additional SEM images of $\mathbf{2}$ taken after slow cooling from the isotropic liquid phase to room temperature (with gold deposition). 


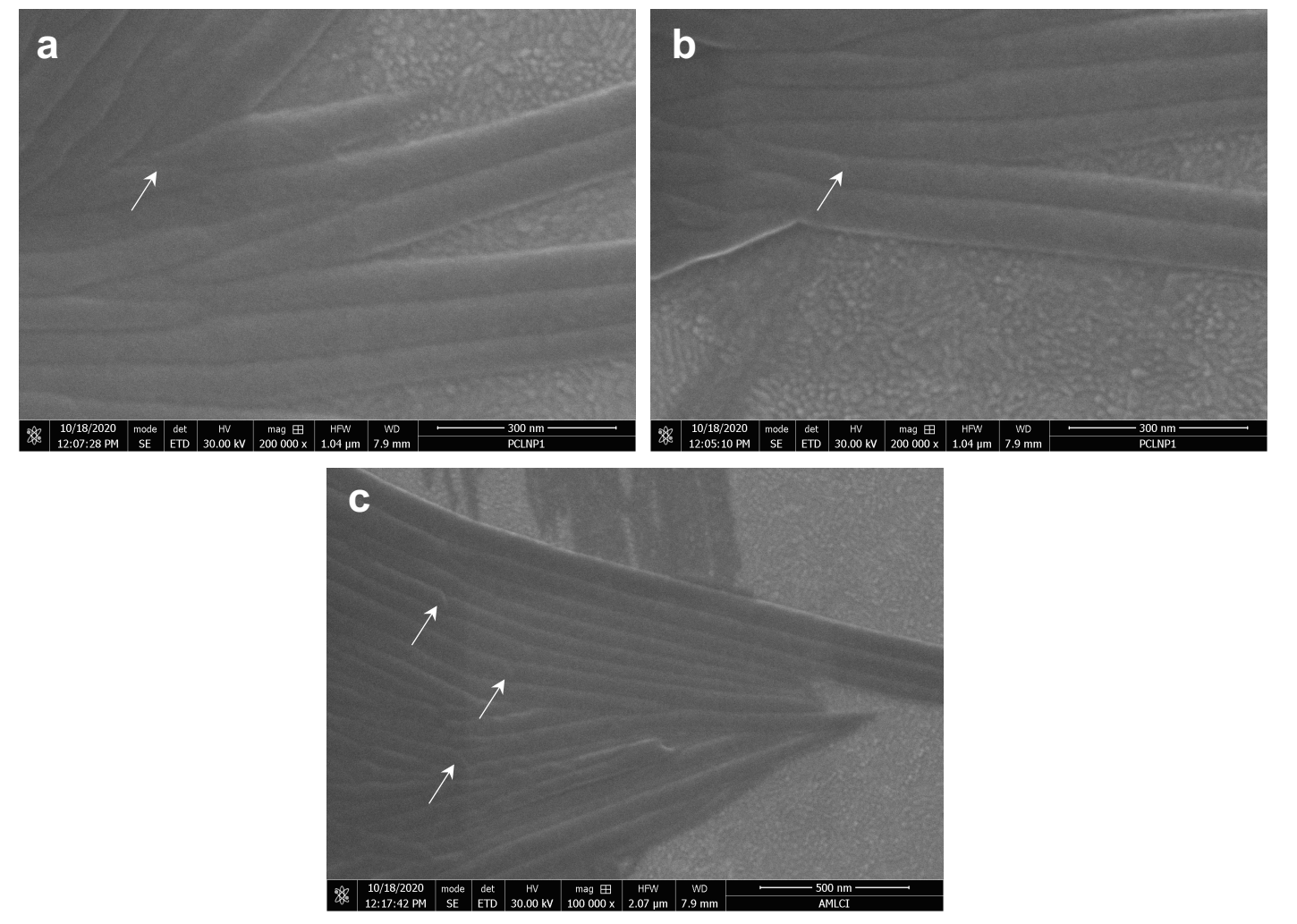

Figure S21. SEM images of $\mathbf{2}$ taken after slow cooling from the isotropic liquid phase to room temperature without any metal deposition: $(\mathrm{a}, \mathrm{b})$ images show at least one area where right-handed heliconical layer wrapping is seen, (c) multiple nanocylinders show consistently right-handed heliconical wrapping, but never in the same nanocylinder, which might indicate a rather large helical pitch $p$. 

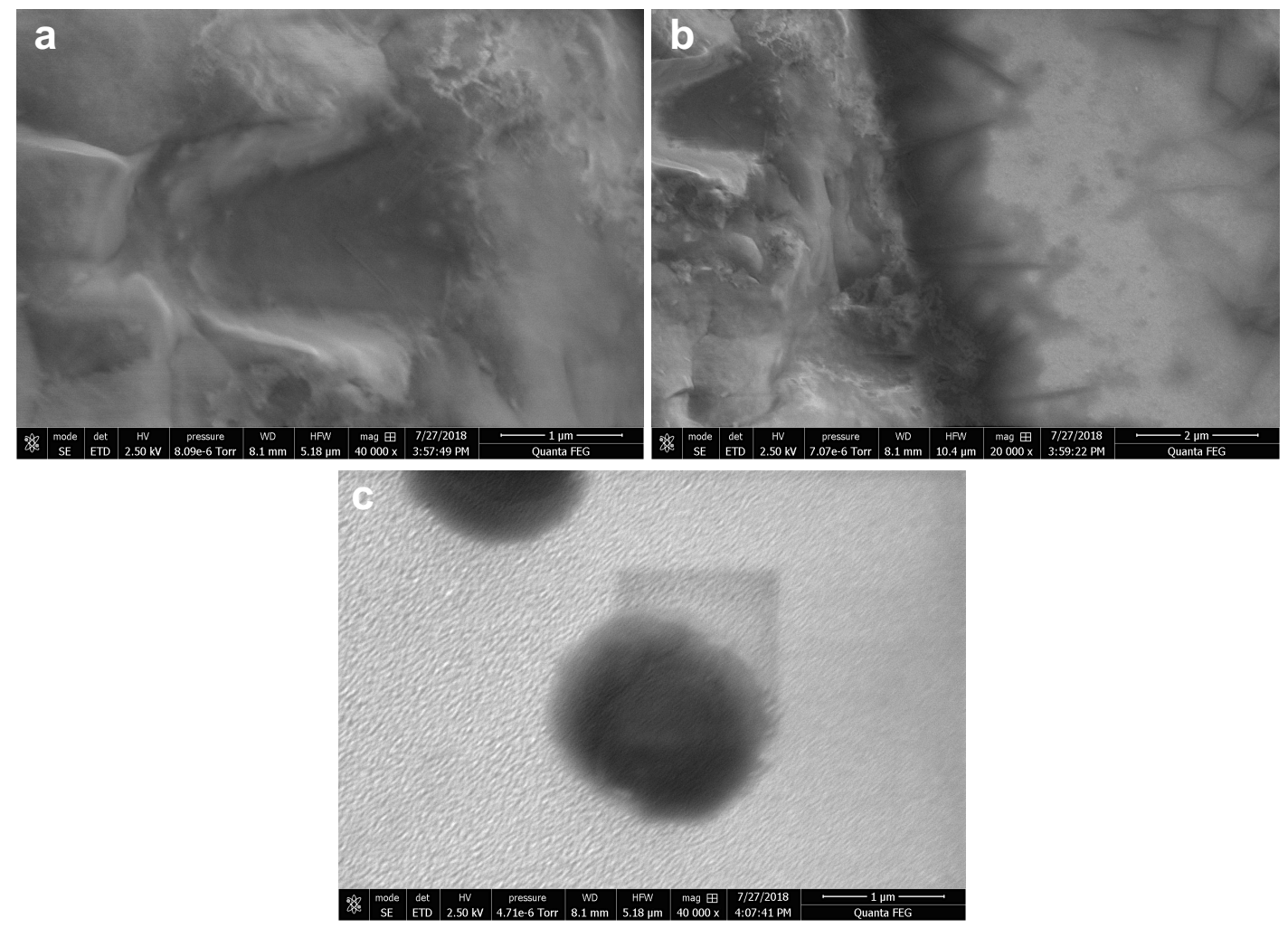

Figure S22. SEM images of $\mathbf{2}$ taken after rapid cooling from the isotropic liquid phase to room temperature: $(a, b)$ images show show textured domains without any indication of nanocylinders typical for the coexistence of the B1 and B4 phase. In many cases, the liquid crystalline phase upon cooling engulfs the nanocylinders and they are only visible on the edges of domains as seen in (b) and on the right of the globular object shown in (c); see also TEM image in Figure 6h in the main article. 


\section{Additional XRD data}

a

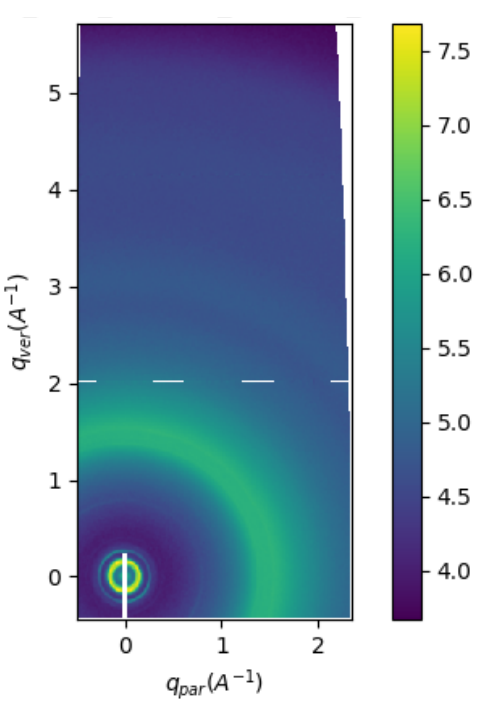

C

\begin{tabular}{|c|c|c|c|}
\hline$q / \AA^{-1}$ & & rel. intensity & index \\
\hline 0.131 & $q_{1}^{a}$ & $3.18 \mathrm{E}+09$ & 001 \\
\hline 0.157 & $q_{2}^{a}$ & $6.86 \mathrm{E}+09$ & 100 \\
\hline 0.265 & $q_{3}^{a}$ & $4.75 \mathrm{E}+09$ & 002 \\
\hline 0.296 & $q_{4}^{a}$ & $2.01 \mathrm{E}+08$ & 102 \\
\hline 0.318 & $q_{5}^{a}$ & $1.93 \mathrm{E}+08$ & 200 \\
\hline 0.372 & $q_{6}^{a}$ & $1.57 \mathrm{E}+08$ & 201 \\
\hline 0.397 & $q_{7}^{a}$ & $1.56 \mathrm{E}+08$ & 202 \\
\hline 0.790 & $q_{8}^{a}$ & $2.52 \mathrm{E}+08$ & 006 \\
\hline
\end{tabular}

b

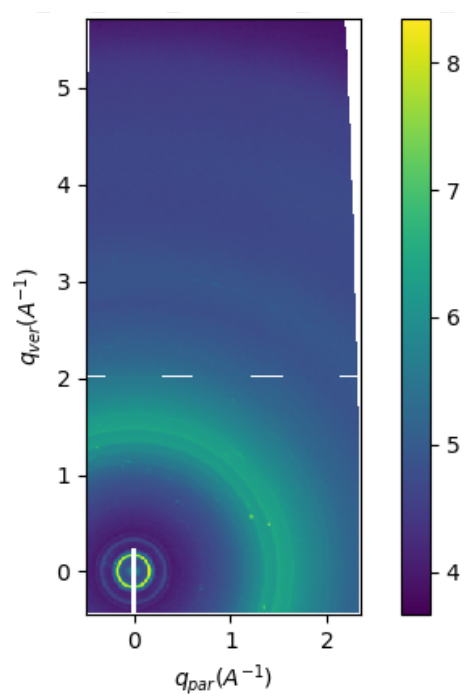

\begin{tabular}{|l|l|l|l|l|}
\hline \multicolumn{1}{|c|}{$/ \AA^{-1}$} & & rel. intensity & \multicolumn{2}{|c}{ index } \\
\hline 0.132 & $q_{1}^{a}$ & $3.75 \mathrm{E}+08$ & 001 & \\
\hline 0.166 & $q_{2}^{a}$ & $2.56 \mathrm{E}+10$ & 100 & \\
\hline 0.197 & $q_{1}^{b}$ & $5.70 \mathrm{E}+08$ & & 101 \\
\hline 0.209 & $q_{3}^{a}$ & $5.89 \mathrm{E}+08$ & 101 & \\
\hline 0.233 & $q_{2}^{b}$ & $1.82 \mathrm{E}+08$ & & 002 \\
\hline 0.255 & $q_{4}^{a}$ & $1.74 \mathrm{E}+08$ & 002 & \\
\hline 0.280 & $q_{3}^{b}$ & $1.88 \mathrm{E}+08$ & & 200 \\
\hline 0.314 & $q_{5}^{a}$ & $2.73 \mathrm{E}+08$ & 102 & \\
\hline 0.333 & $q_{6}^{a}$ & $4.17 \mathrm{E}+08$ & 200 & \\
\hline 0.351 & $q_{4}^{b}$ & $0.35 \mathrm{E}+08$ & & 202 \\
\hline 0.384 & $q_{5}^{b}$ & $2.11 \mathrm{E}+08$ & & 103 \\
\hline 0.394 & $q_{7}^{a}$ & $1.66 \mathrm{E}+08$ & 003 & \\
\hline 0.459 & $q_{6}^{b}$ & $1.65 \mathrm{E}+08$ & & 203 \\
\hline 0.499 & $q_{7}^{b}$ & $0.50 \mathrm{E}+08$ & & 302 \\
\hline 0.512 & $q_{8}^{a}$ & $1.73 \mathrm{E}+08$ & 301 & \\
\hline 0.616 & $q_{8}^{b}$ & $2.01 \mathrm{E}+08$ & & 304 \\
\hline 0.696 & $q_{9}^{b}$ & $2.35 \mathrm{E}+08$ & & 006 \\
\hline 0.709 & $q_{9}^{a}$ & $2.46 \mathrm{E}+08$ & 402 & \\
\hline 0.844 & $q_{10}^{a}$ & $3.09 \mathrm{E}+08$ & 501 & \\
\hline & & & & \\
\hline
\end{tabular}

Figure S23. 2D XRD pattern obtained for compound 2 on: (a) slow cooling and (b) fast cooling. Momentum transfer $(q)$, relative intensities, and Miller indices for each phase upon: (c) slow cooling and (d) fast cooling - all from BNL beamline data. 


\section{Section S4. Additional Data for Compound 3}

\section{DSC data}
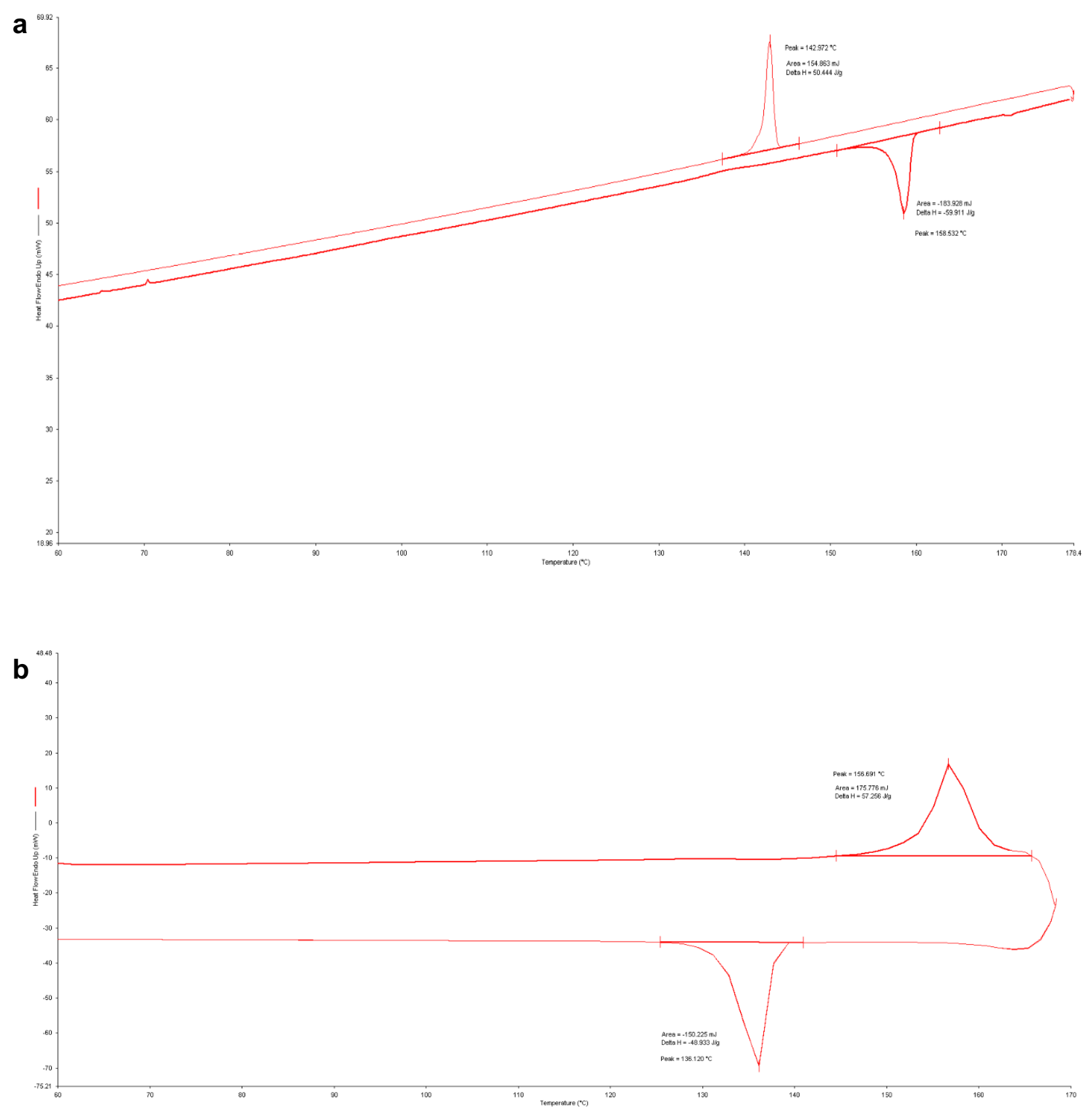

Figure S24. DSC thermograms of compound 3 at a heating/cooling rate of (a) $5{ }^{\circ} \mathrm{C} \min ^{-1}$ and (b) $50{ }^{\circ} \mathrm{C}$ $\min ^{-1}$ : glass transitions are here not detectable, and the $1^{\text {st }}$ and $2^{\text {nd }}$ heating/cooling run are, as for compounds 1 and 2, quasi identical. Hence, only the $2^{\text {nd }}$ heating/cooling runs are shown since these data are used in Scheme 1 (main article) and Table S3 below. 
Table S3. DSC data for compound 3 at both heating/cooling rates $-2^{\text {nd }}$ heating/cooling run.

\begin{tabular}{lccc}
\hline $\begin{array}{l}\text { Rate } \\
{\left[{ }^{\circ} \mathrm{min}^{-1}\right]}\end{array}$ & $\begin{array}{l}\text { Run } \\
\text { on Heating }{ }^{\circ} \mathrm{C}[\mathrm{kJ} / \mathrm{mol}]\end{array}$ & $\begin{array}{l}\text { Transition Temperature [Enthalpy] } \\
\text { on Cooling }{ }^{\circ} \mathrm{C}[\mathrm{kJ} / \mathrm{mol}]\end{array}$ \\
\hline 5 & $2^{\text {nd }}$ & $143.1[41.9]$ & $158.6[-49.8]$ \\
\hline 50 & $2^{\text {nd }}$ & $156.7[47.6]$ & $136.1[-40.7]$ \\
\hline
\end{tabular}

\section{Additional SEM and TEM images}
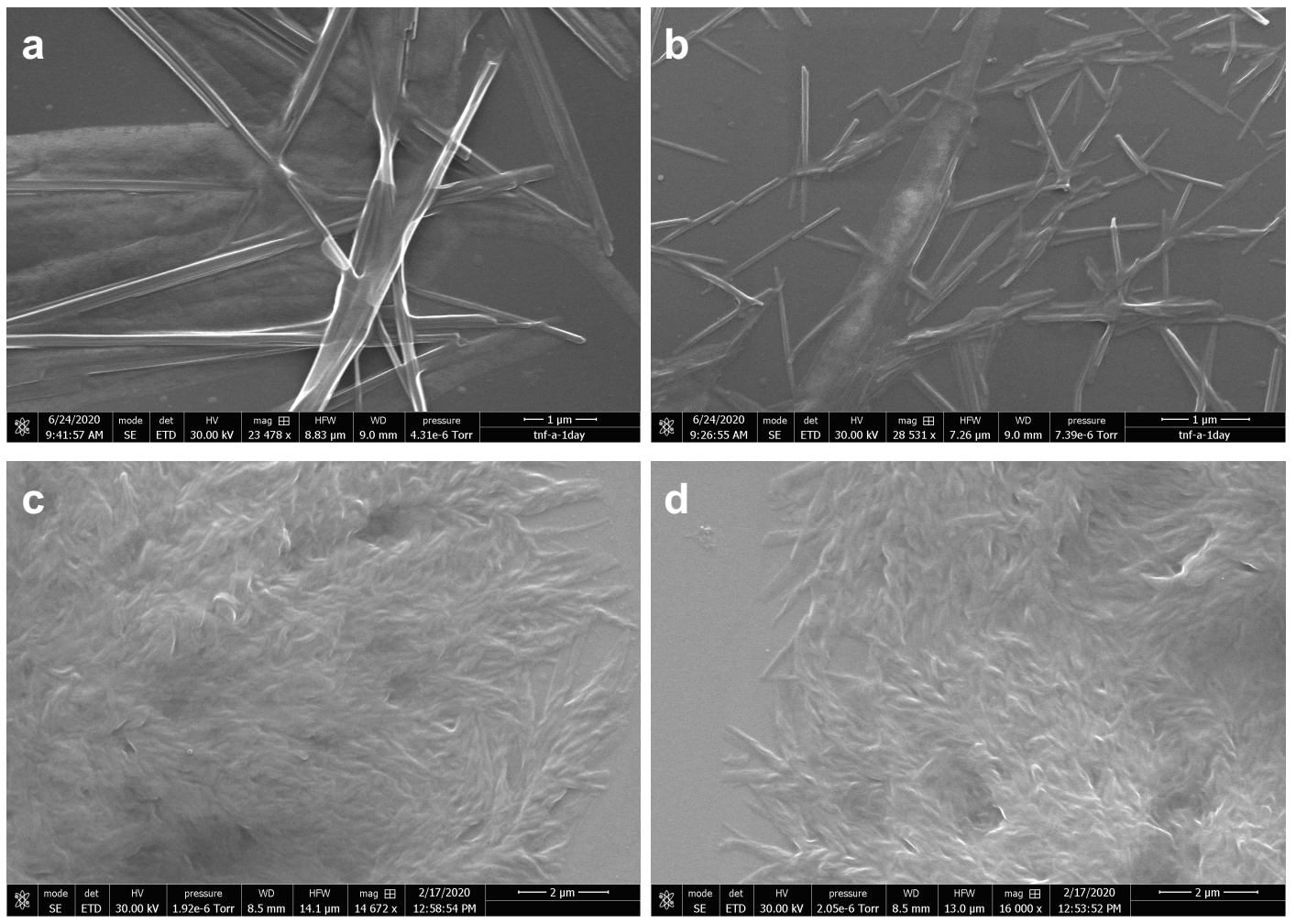

Figure S25. SEM images of 3: $(a, b)$ taken after slow cooling from the isotropic liquid phase to room temperature, showing mostly FNRs and $(c, d)$ taken after rapid cooling from the isotropic liquid phase to room temperature showing both left and righ-handed HNFs. 

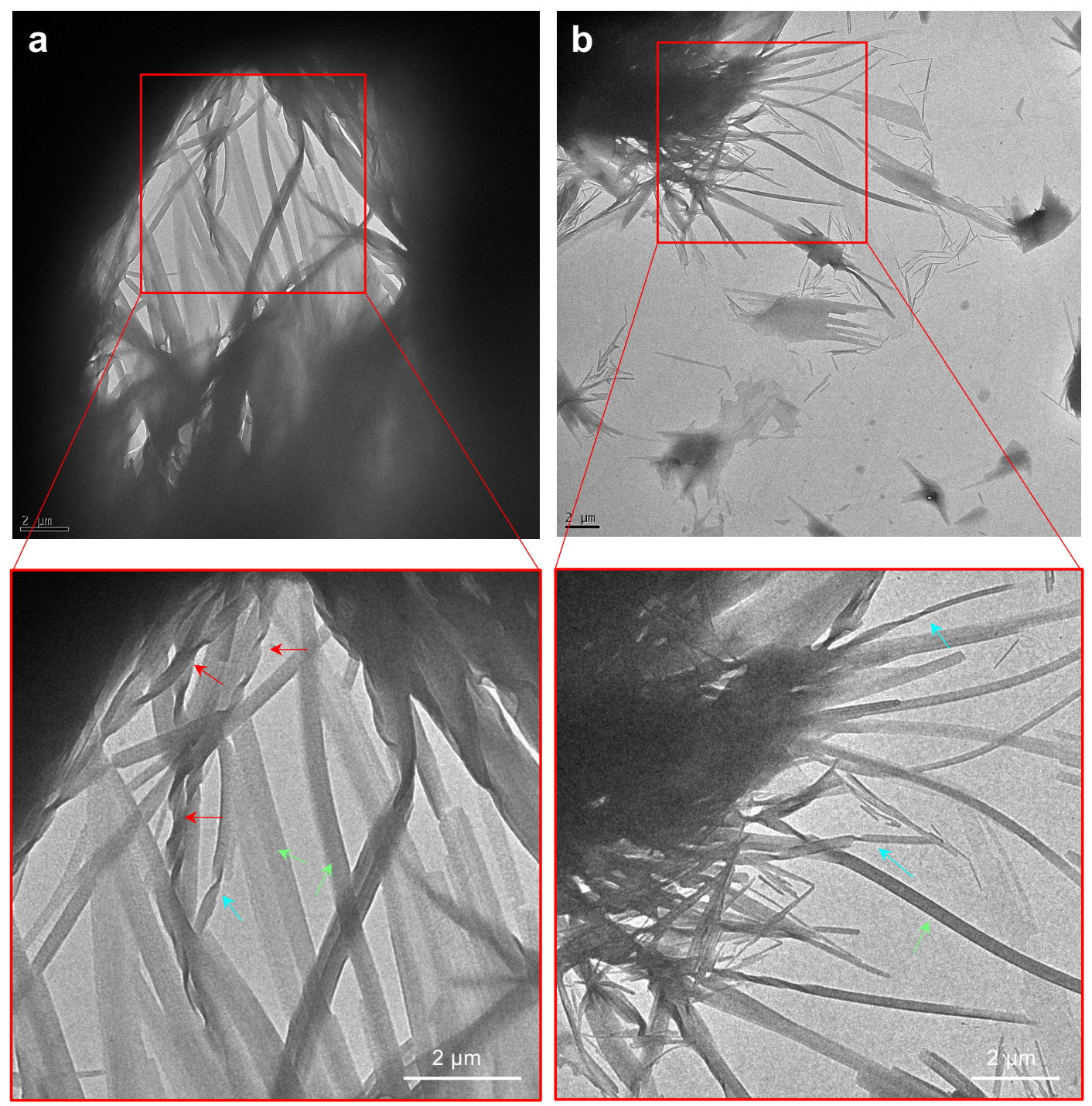

Figure S26. TEM images of 3: $(a, b)$ taken after slow cooling from the isotropic liquid phase to room temperature, showing flat (green arrows), occasionally twisted (turquois arrows) and fully twisted nanoribbons (red arrows). Scale bars in (a) and (b) $2 \mu \mathrm{m}$. 

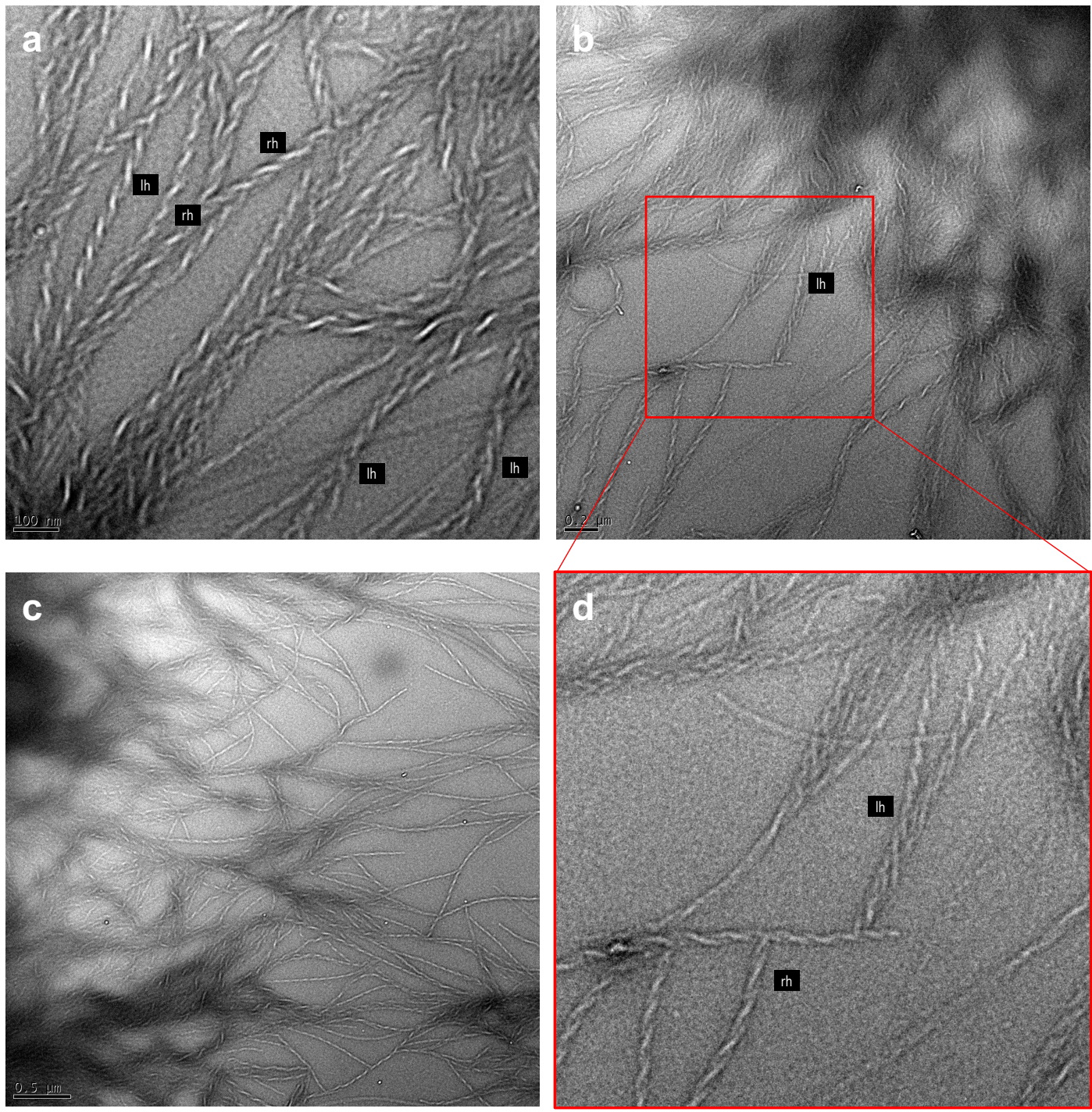

Figure S27. TEM images of 3: $(a, b)$ taken after rapid cooling from the isotropic liquid phase to room temperature, showing both left- and right-handed HNFs (frequently adjacent to one another). Scale bars: (a) $100 \mathrm{~nm}$, (b) $200 \mathrm{~nm}$. 


\section{Additional XRD data}

a

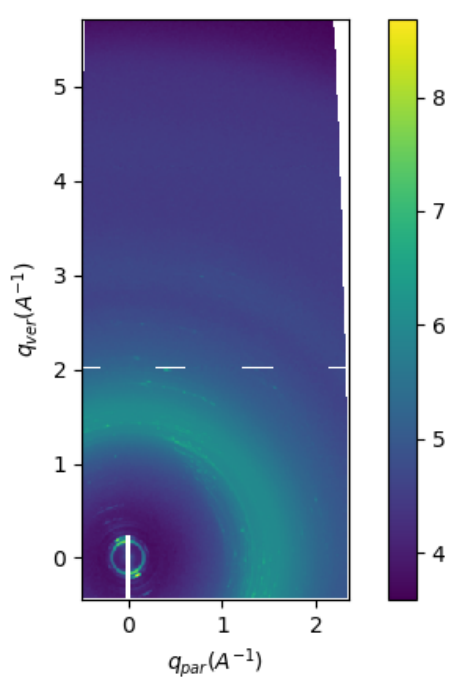

C

\begin{tabular}{|c|c|c|c|}
\hline$q / \AA^{-1}$ & & rel. intensity & index \\
\hline 0.141 & $q_{1}^{a}$ & $1.56 \mathrm{E}+08$ & 001 \\
\hline 0.171 & $q_{2}^{a}$ & $1.61 \mathrm{E}+09$ & 100 \\
\hline 0.218 & $q_{3}^{a}$ & $2.49 \mathrm{E}+08$ & 101 \\
\hline 0.268 & $q_{4}^{a}$ & $1.28 \mathrm{E}+08$ & 010 \\
\hline 0.284 & $q_{5}^{a}$ & $1.18 \mathrm{E}+08$ & 002 \\
\hline 0.308 & $q_{6}^{a}$ & $1.17 \mathrm{E}+08$ & 011 \\
\hline 0.313 & $q_{7}^{a}$ & $1.19 \mathrm{E}+08$ & 110 \\
\hline 0.343 & $q_{8}^{a}$ & $1.39 \mathrm{E}+08$ & 200 \\
\hline 0.367 & $q_{9}^{a}$ & $1.28 \mathrm{E}+08$ & 201 \\
\hline 0.376 & $q_{10}^{a}$ & $1.32 \mathrm{E}+08$ & 012 \\
\hline 0.422 & $q_{11}^{a}$ & $1.30 \mathrm{E}+08$ & 003 \\
\hline 0.435 & $q_{12}^{a}$ & $1.34 \mathrm{E}+08$ & 210 \\
\hline
\end{tabular}

b

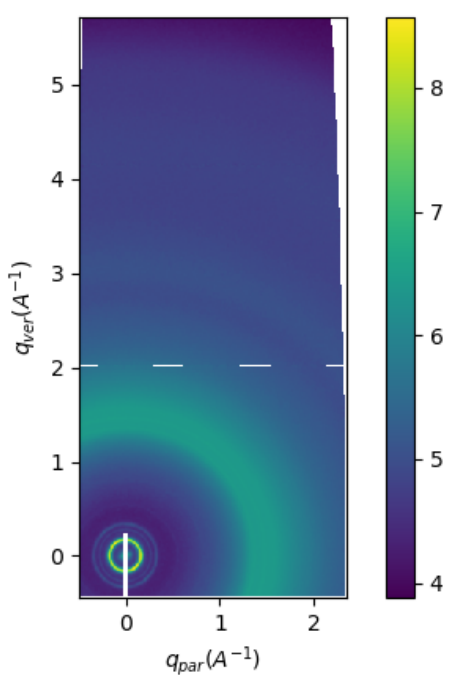

d

\begin{tabular}{|c|c|c|c|}
\hline$q / \AA^{-1}$ & & $\begin{array}{c}\text { Rel. } \\
\text { Intensity }\end{array}$ & Index \\
\hline 0.136 & $q_{1}^{b}$ & $8.04 \mathrm{E}+08$ & 001 \\
\hline 0.166 & $q_{2}^{b}$ & $1.93 \mathrm{E}+08$ & 100 \\
\hline 0.2428 & $q_{3}^{b}$ & $2.60 \mathrm{E}+08$ & 010 \\
\hline 0.2721 & $q_{4}^{b}$ & $3.64 \mathrm{E}+08$ & 002 \\
\hline 0.3326 & $q_{5}^{b}$ & $4.10 \mathrm{E}+08$ & 200 \\
\hline 0.3736 & $q_{6}^{b}$ & $2.05 \mathrm{E}+08$ & 012 \\
\hline 0.4113 & $q_{7}^{b}$ & $1.98 \mathrm{E}+08$ & 210 \\
\hline 0.548 & $q_{8}^{b}$ & $2.28 \mathrm{E}+08$ & 022 \\
\hline 0.813 & $q_{9}^{b}$ & $3.36 \mathrm{E}+08$ & 006 \\
\hline
\end{tabular}

e

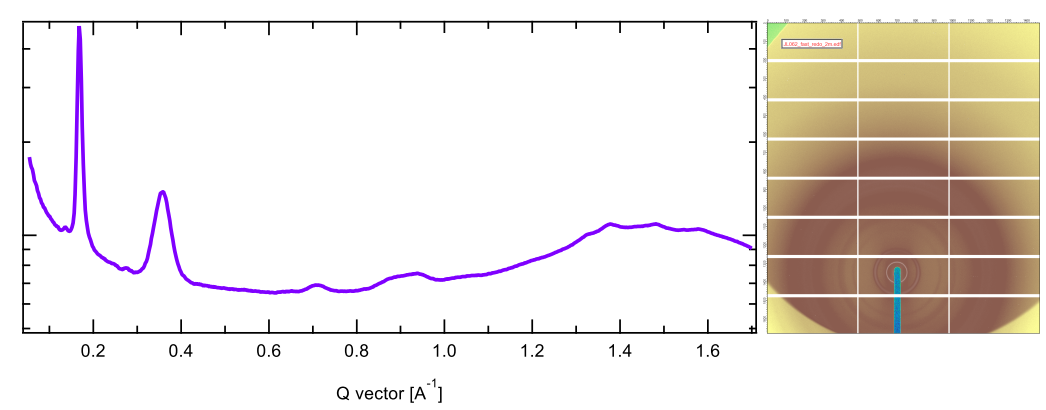

Figure S28. 2D XRD pattern obtained for compound 3 on: (a) slow cooling and (b) fast cooling. Momentum transfer ( $q$ ), Miller indices, and relative intensities upon: (c) slow cooling and (d) fast cooling - all from BNL beamline. (e) Complementary ALS beamline data for compound $\mathbf{3}$ taken upon fast cooling from the isotropic liquid phase (used to further support the $\mathrm{B} 4 \mathrm{HNF}_{\bmod 2}$ phase assignment ${ }^{1}$; here $q_{1}^{\mathrm{b}}=0.136 \AA^{-1}$ is barely visible). 


\section{Section S5. Additional Data for Compound 4}

\section{Additional POM images}

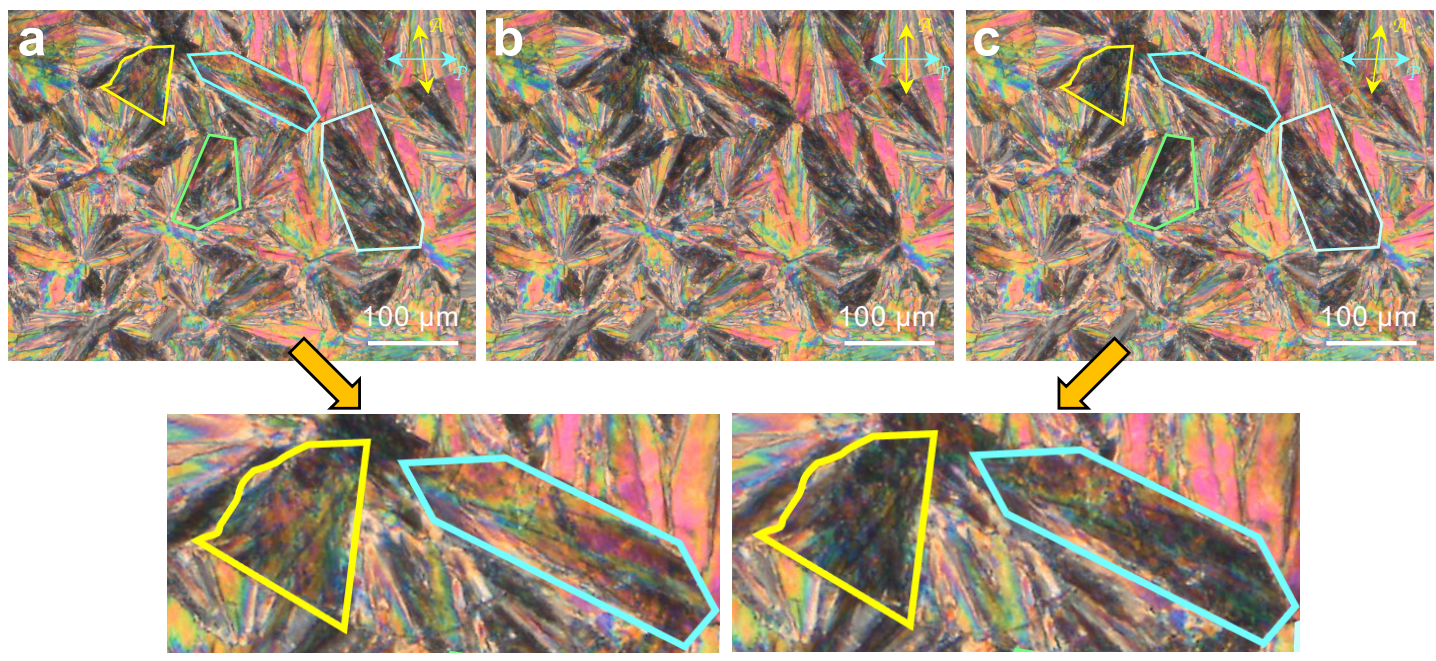

Figure S29. POM photomicrographs for compound $\mathbf{4}$ after heating to the isotropic liquid phase and subsequent rapid cooling to room temperature between untreated glass slides at a rate of $\sim 50{ }^{\circ} \mathrm{C} \min ^{-1}$ : (a) $-8^{\circ}$ decrossed polarizers, (b) crossed polarizers, and (c) $+8^{\circ}$ decrossed polarizers. Minor differences in the brightness (color, birefringence) of the darker domains is highlighted for some by colored polygons (magnified again below) in the $-8^{\circ}$ and $+8^{\circ}$ decrossed polarizer images.
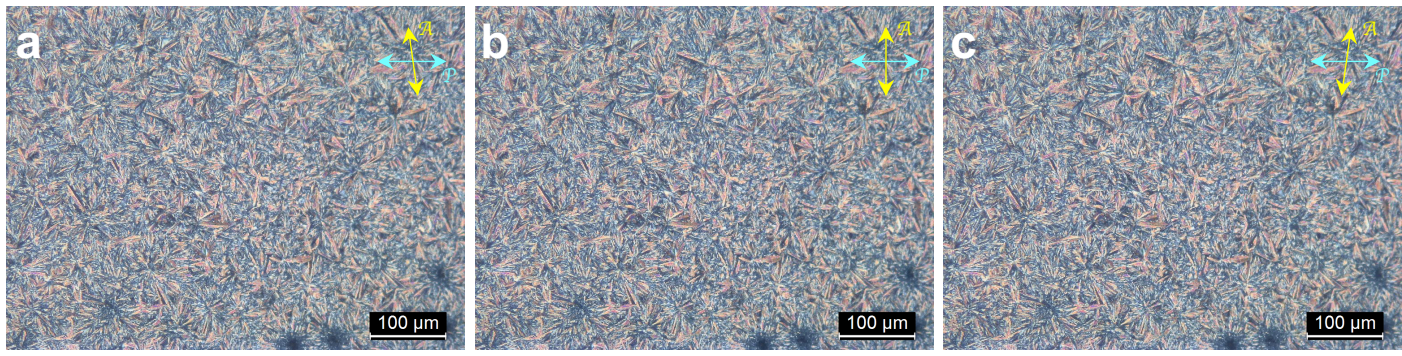

Figure S30. POM photomicrographs for compound $\mathbf{4}$ after heating to the isotropic liquid phase and subsequent rapid cooling to room temperature between the CD quartz substrates (cell gap: 10 micron) at a rate of $\sim 50{ }^{\circ} \mathrm{C} \min ^{-1}$ : (a) $-8^{\circ}$ decrossed polarizers, (b) crossed polarizers, and (c) $+8^{\circ}$ decrossed polarizers. 


\section{DSC data}
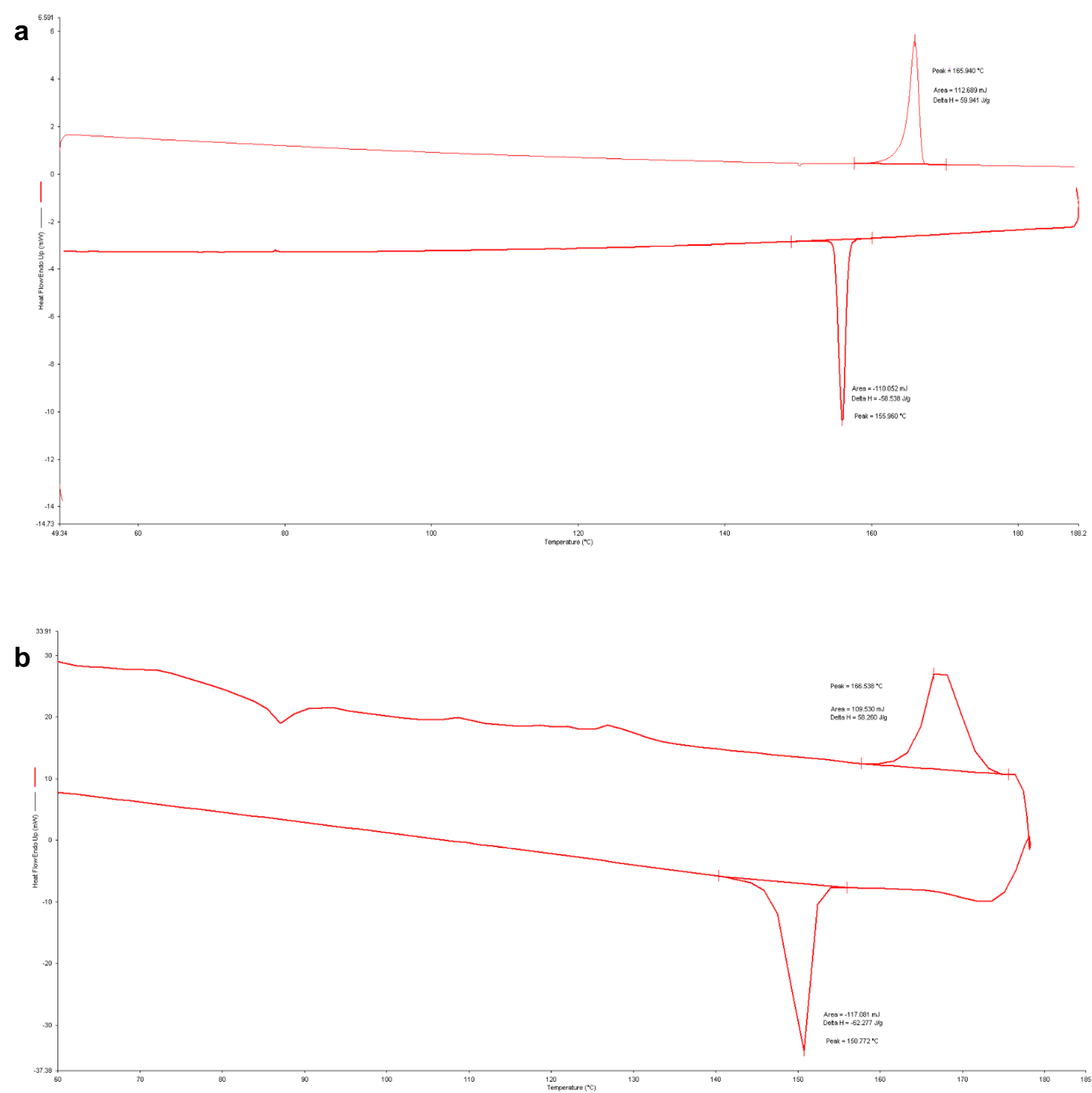

Figure S31. DSC thermograms of compound 4 at a heating/cooling rate of (a) $5{ }^{\circ} \mathrm{C} \min ^{-1}$ and (b) $50{ }^{\circ} \mathrm{C}$ $\min ^{-1}$ : glass transitions are here not detectable, and the $1^{\text {st }}$ and $2^{\text {nd }}$ heating/cooling run are, as for compounds $1-3$, quasi identical. Hence, only the $2^{\text {nd }}$ heating/cooling runs are shown since these data are used in Scheme 1 (main article) and Table S4 below. 
Table S4. DSC data for compound 4 at both heating/cooling rates $-2^{\text {nd }}$ heating/cooling run.

\begin{tabular}{lrrc}
\hline $\begin{array}{l}\text { Rate } \\
{\left[{ }^{\circ} \mathrm{C} \mathrm{min}^{-1}\right]}\end{array}$ & $\begin{array}{l}\text { Run } \\
\text { on Heating }{ }^{\circ} \mathrm{C}[\mathrm{kJ} / \mathrm{mol}]\end{array}$ & $\begin{array}{l}\text { Transition Temperature [Enthalpy] } \\
\text { on Cooling }{ }^{\circ} \mathrm{C}[\mathrm{kJ} / \mathrm{mol}]\end{array}$ \\
\hline 5 & $2^{\text {nd }}$ & $165.9[49.0]$ & $155.4[-47.8]$ \\
\hline 50 & $2^{\text {nd }}$ & $168.2[47.6]$ & $150.8[-50.9]$ \\
\hline
\end{tabular}

\section{Additional TEM images}
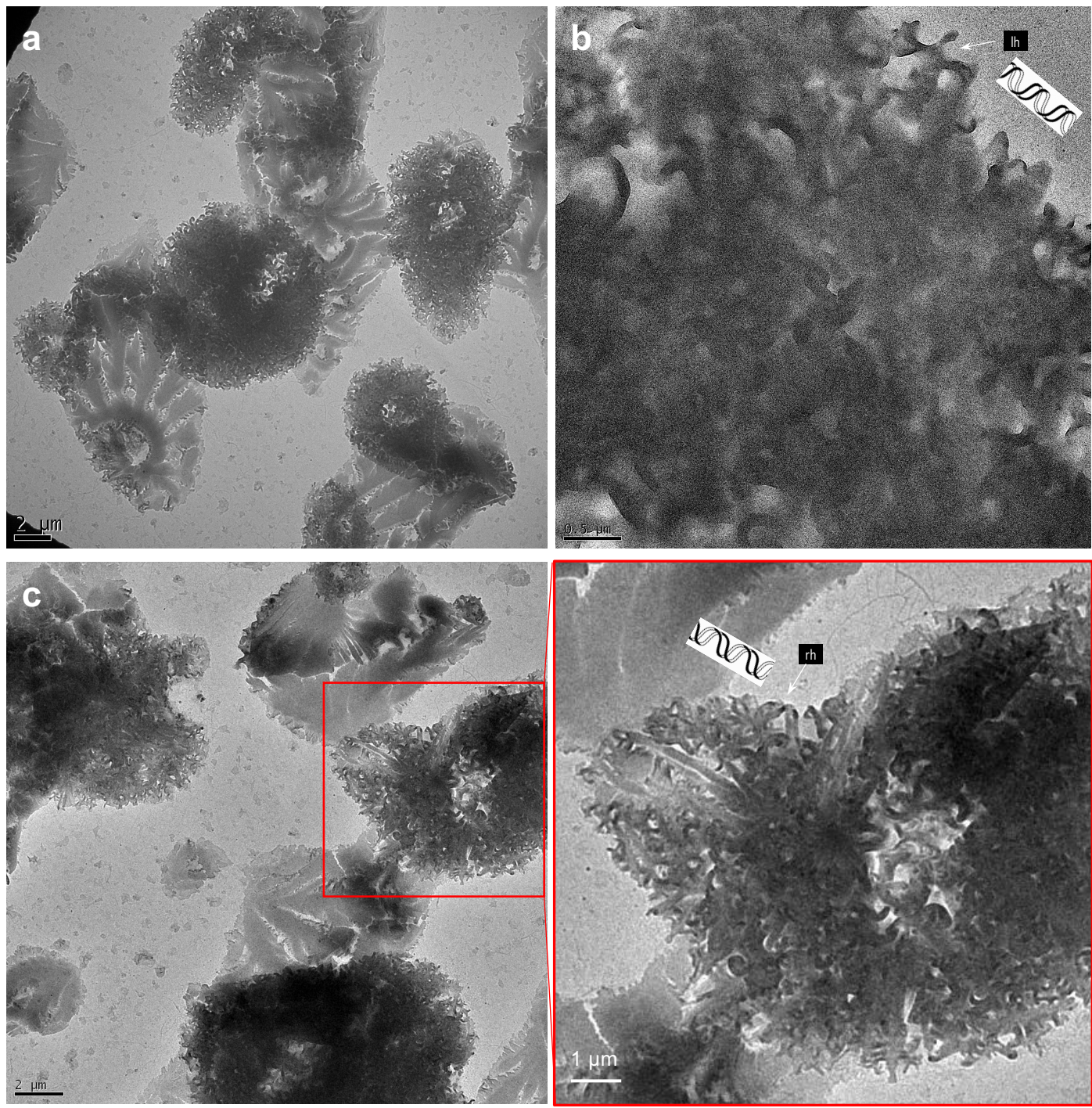

Figure S32. TEM images of 4: $(\mathrm{a}-\mathrm{c})$ taken after rapid cooling from the isotropic liquid phase to room temperature, showing coexistence of smooth domains (from the columnar phase) and $\mathrm{H} \mu \mathrm{F}$ domains with either left- or right-handed twist (see inset models). It is often dfficult to see if the H $\mu \mathrm{Fs}$ have consistently identical handedness throughout due to the severe bundling of the $\mathrm{H} \mu \mathrm{Fs}$ in these domains. Scale bars: (a, c) $2 \mu \mathrm{m}$, (b) $500 \mathrm{~nm}$. 


\section{Additional XRD data}

a

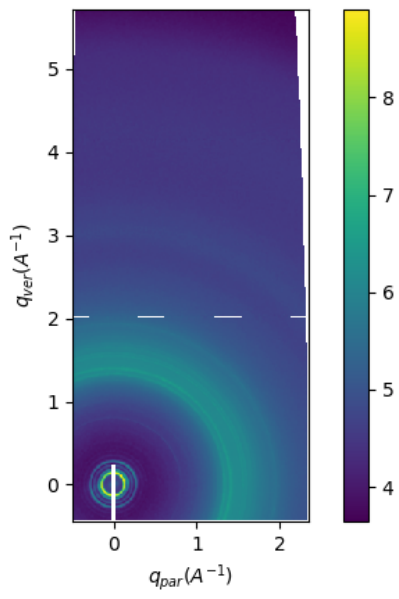

C

\begin{tabular}{|c|c|c|c|}
\hline$q / \AA^{-1}$ & & rel. intensity & index \\
\hline 0.138 & $q_{1}^{a}$ & $3.67 \mathrm{E}+10$ & 001 \\
\hline 0.177 & $q_{2}^{a}$ & $2.85 \mathrm{E}+09$ & 100 \\
\hline 0.229 & $q_{3}^{a}$ & $4.73 \mathrm{E}+08$ & 101 \\
\hline 0.276 & $q_{4}^{a}$ & $1.70 \mathrm{E}+09$ & 002 \\
\hline 0.300 & $q_{5}^{a}$ & $2.14 \mathrm{E}+08$ & 010 \\
\hline 0.347 & $q_{6}^{a}$ & $1.70 \mathrm{E}+08$ & 200 \\
\hline 0.372 & $q_{7}^{a}$ & $1.39 \mathrm{E}+08$ & 111 \\
\hline 0.384 & $q_{8}^{a}$ & $1.30 \mathrm{E}+08$ & 201 \\
\hline 0.4035 & $q_{9}^{a}$ & $1.78 \mathrm{E}+08$ & 003 \\
\hline 0.440 & $q_{10}^{a}$ & $1.34 \mathrm{E}+08$ & 112 \\
\hline 0.460 & $q_{11}^{a}$ & $1.40 \mathrm{E}+08$ & 103 \\
\hline 0.517 & $q_{12}^{a}$ & $1.46 \mathrm{E}+08$ & 300 \\
\hline 0.533 & $q_{13}^{a}$ & $1.58 \mathrm{E}+08$ & 203 \\
\hline 0.547 & $q_{14}^{a}$ & $1.54 \mathrm{E}+08$ & 301 \\
\hline 0.676 & $q_{15}^{a}$ & $1.92 \mathrm{E}+08$ & 114 \\
\hline 0.8009 & $q_{16}^{a}$ & $2.58 \mathrm{E}+08$ & 304 \\
\hline 0.814 & $q_{17}^{a}$ & $2.89 \mathrm{E}+08$ & 006 \\
\hline
\end{tabular}

b

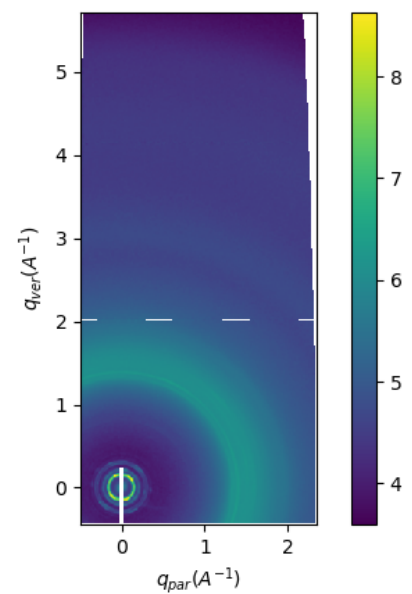

d

\begin{tabular}{|c|c|c|c|c|}
\hline$q / \AA^{-1}$ & & rel. intensity & \multicolumn{2}{|c|}{ index } \\
\hline 0.148 & $q_{1}^{b}$ & $1.71 \mathrm{E}+08$ & 001 & \\
\hline 0.157 & $q_{2}^{b}$ & $4.84 \mathrm{E}+09$ & 100 & \\
\hline 0.160 & $q_{1}^{c}$ & $1.23 \mathrm{E}+08$ & & 101 \\
\hline 0.218 & $q_{3}^{b}$ & $4.70 \mathrm{E}+08$ & 101 & \\
\hline 0.226 & $q_{2}^{c}$ & $2.57 \mathrm{E}+08$ & & 002 \\
\hline 0.228 & $q_{3}^{c}$ & $2.31 \mathrm{E}+08$ & & 200 \\
\hline 0.246 & $q_{4}^{c}$ & $2.08 \mathrm{E}+08$ & & 102 \\
\hline 0.284 & $q_{4}^{b}$ & $3.60 \mathrm{E}+08$ & 002 & \\
\hline 0.314 & $q_{5}^{b}$ & $3.09 \mathrm{E}+08$ & 200 & \\
\hline 0.322 & $q_{5}^{c}$ & $2.20 \mathrm{E}+08$ & & 202 \\
\hline 0.357 & $q_{6}^{b}$ & $2.21 \mathrm{E}+08$ & 201 & \\
\hline 0.362 & $q_{6}^{c}$ & $1.78 \mathrm{E}+08$ & & 103 \\
\hline 0.409 & $q_{7}^{b}$ & $1.58 \mathrm{E}+08$ & 010 & \\
\hline 0.439 & $q_{8}^{b}$ & $1.52 \mathrm{E}+08$ & 202 & \\
\hline 0.447 & $q_{9}^{b}$ & $1.34 \mathrm{E}+08$ & 003 & \\
\hline 0.489 & $q_{10}^{b}$ & $1.55 \mathrm{E}+08$ & 301 & \\
\hline 0.497 & $q_{7}^{c}$ & $1.56 \mathrm{E}+08$ & & 204 \\
\hline
\end{tabular}
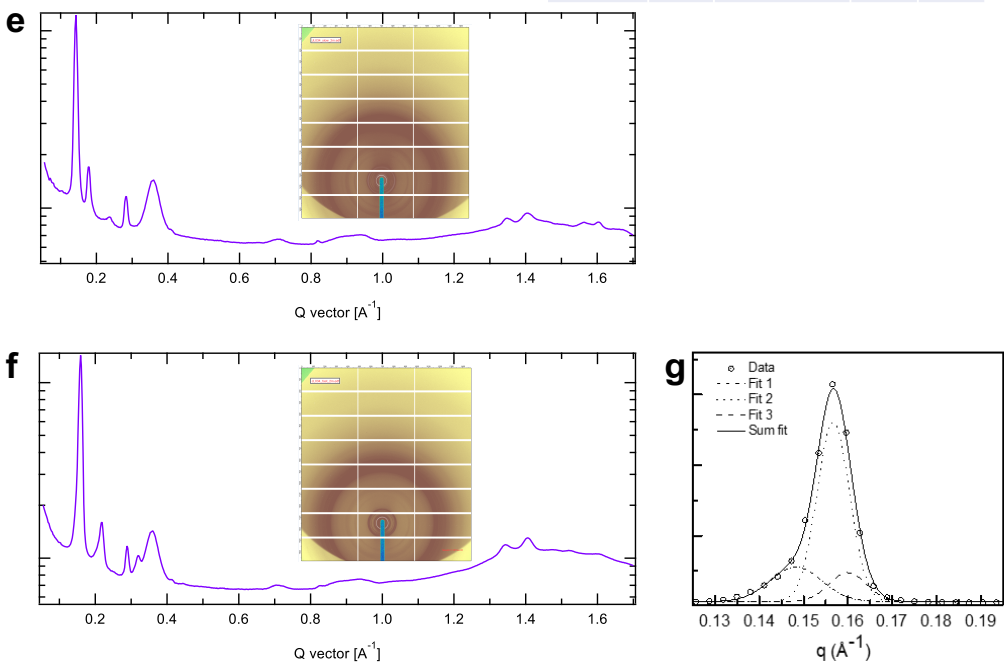

Figure S33. 2D XRD pattern obtained for compound 4 on: (a) slow cooling and (b) fast cooling. Momentum transfer $(q)$, indexing, and relative intensities on: (c) slow cooling and (d) fast cooling; including indexing for the $\mathrm{Col}_{\mathrm{r}}-c 2 \mathrm{~mm}$ phase - all from BNL beamline. Complementary ALS beamline data for compound $\mathbf{4}$ taken: (e) upon slow cooling and (f) upon fast cooling from the isotropic liquid phase (used to further support the $\mathrm{B} 1 \mathrm{Col}_{\mathrm{r}}-c 2 m m$ phase assignment). (g) Deconvolution of $q_{1}^{\mathrm{b}}$ and $q_{2}^{\mathrm{b}}$ (fast cooling, BNL dataset). 


\section{Section S6. Additional Data for Compound 5}

\section{DSC data}
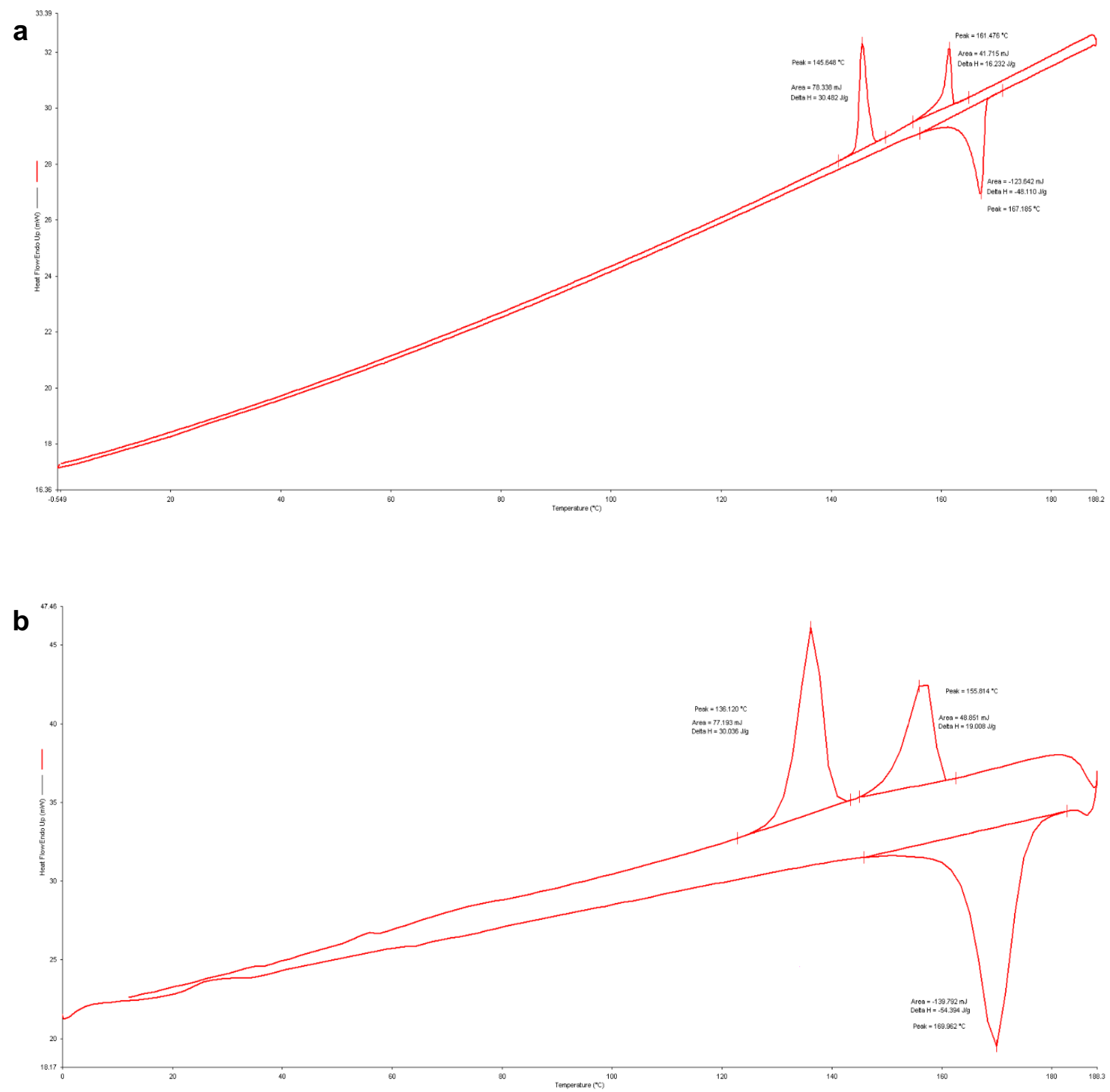

Figure S34. DSC thermograms of compound 5 at a heating/cooling rate of (a) $5{ }^{\circ} \mathrm{C} \min ^{-1}$ and (b) $50{ }^{\circ} \mathrm{C}$ $\min ^{-1}$ : glass transitions are here not detectable, and the $1^{\text {st }}$ and $2^{\text {nd }}$ heating/cooling run are, as for compounds $1-4$, quasi identical. Hence, only the $2^{\text {nd }}$ heating/cooling runs are shown since these data are used in Scheme 1 (main article) and Table S5 below. 
Table S5. DSC data for compound 5 at both heating/cooling rates $-2^{\text {nd }}$ heating/cooling run.

\begin{tabular}{|c|c|c|c|}
\hline $\begin{array}{l}\text { Rate } \\
{\left[{ }^{\circ} \mathrm{C} \min ^{-1}\right]}\end{array}$ & Run & $\begin{array}{l}\text { Transition Temperature [Enthalpy] } \\
\text { on Heating }{ }^{\circ} \mathrm{C}[\mathrm{kJ} / \mathrm{mol}]\end{array}$ & $\begin{array}{l}\text { Transition Temperature [Enthalpy] } \\
\text { on Cooling }{ }^{\circ} \mathrm{C}[\mathrm{kJ} / \mathrm{mol}]\end{array}$ \\
\hline 5 & $2^{\text {nd }}$ & $\begin{array}{l}\mathrm{Cr} \rightarrow \text { LC phase } 146.2[24.9] \\
\text { LC phase } \rightarrow \text { Iso } 161.2[13.3]\end{array}$ & Iso $\rightarrow$ LC phase $166.9[-39.3]$ \\
\hline 50 & $2^{\text {nd }}$ & $\begin{array}{l}\mathrm{Cr} \rightarrow \text { LC phase } 137.6[24.5] \\
\text { LC phase } \rightarrow \text { Iso } 157.3[15.5]\end{array}$ & Iso $\rightarrow$ LC phase $168.2[-44.5]$ \\
\hline
\end{tabular}

\section{Additional POM images}
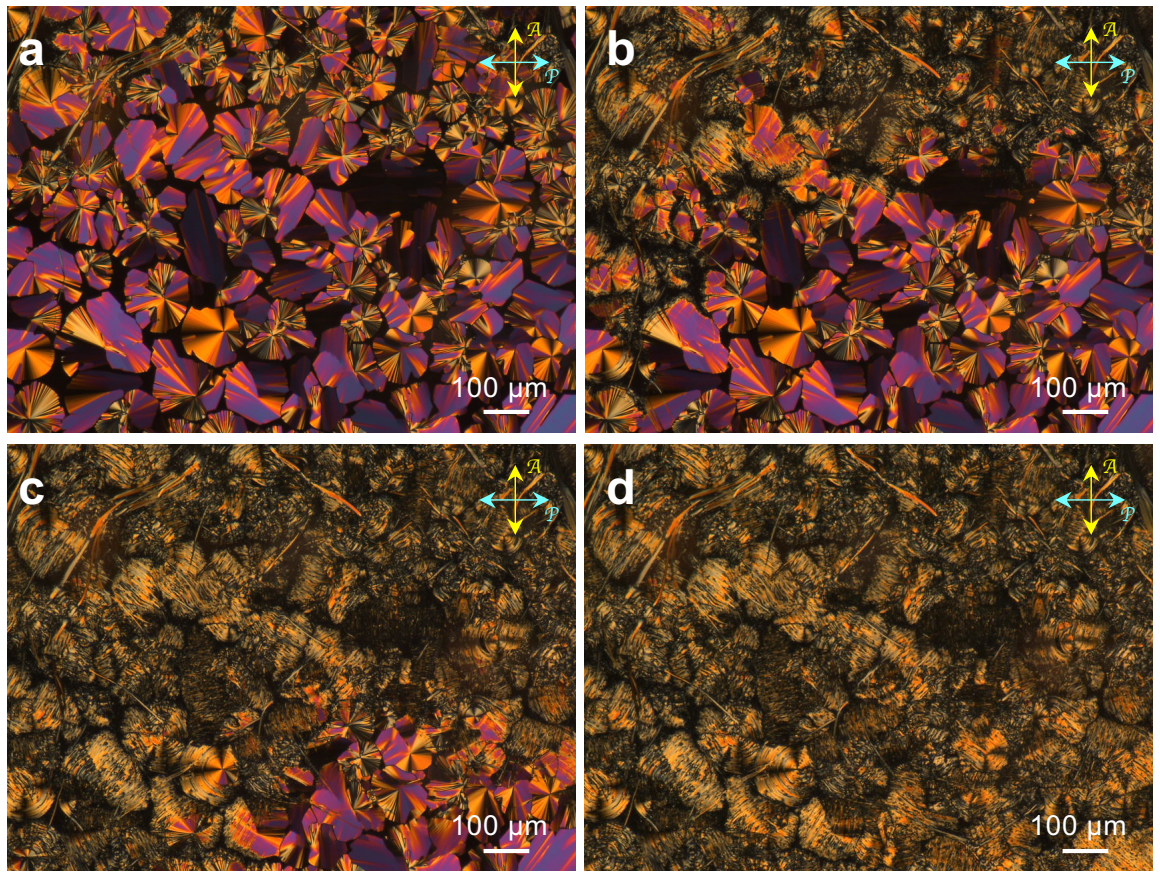

Figure S35. POM photomicrographs (crossed polarizers) for compound $\mathbf{5}$ after heating to the isotropic liquid phase and subsequent slow cooling to room temperature between untreated glass substrates at a rate of $5{ }^{\circ} \mathrm{C} \mathrm{min}-1$ : (a) at $166^{\circ} \mathrm{C}$, (b) at $160{ }^{\circ} \mathrm{C}$, (c) at $157^{\circ} \mathrm{C}$, and (d) at $154{ }^{\circ} \mathrm{C}$. 


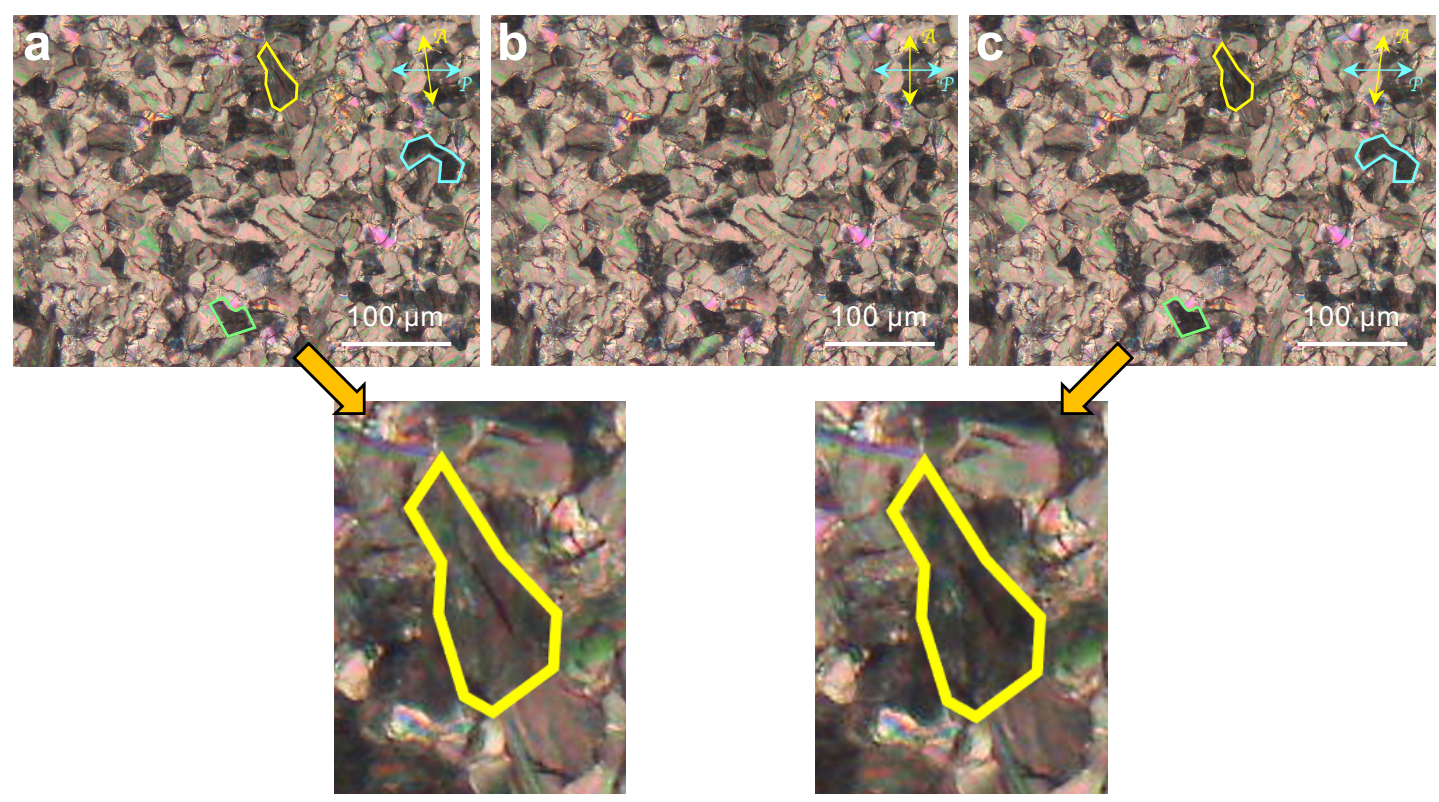

Figure S36. POM photomicrographs for compound $\mathbf{5}$ after heating to the isotropic liquid phase and subsequent rapid cooling to room temperature between untreated glass slides at a rate of $\sim 50{ }^{\circ} \mathrm{C} \mathrm{min}{ }^{-1}$ : (a) $-8^{\circ}$ decrossed polarizers, (b) crossed polarizers, and (c) $+8^{\circ}$ decrossed polarizers. Minor differences in the brightness (color, birefringence) of the darker domains is highlighted for some by colored polygons (enlarged section are displayed again below) in the $-8^{\circ}$ and $+8^{\circ}$ decrossed polarizer images. 


\section{Additional SEM and TEM images}
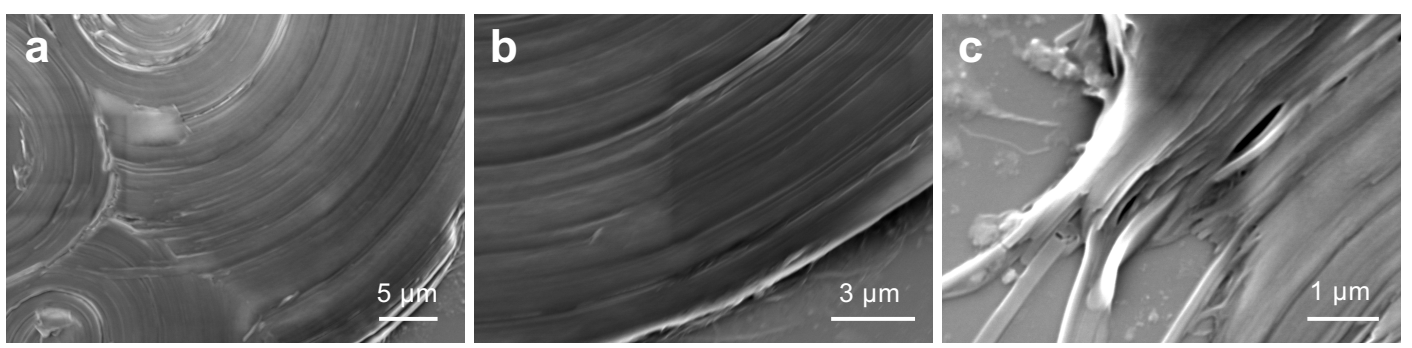

Figure S37. SEM images of 5 taken after slow cooling from the isotropic liquid phase to room temperature: (a) overlapping spherulitic domains, (b) close up on the grooves, and (c) close up on the periphery. These domains seen by SEM resemble vinyl records based on the circular shape and the presence of clearly visible grooves.
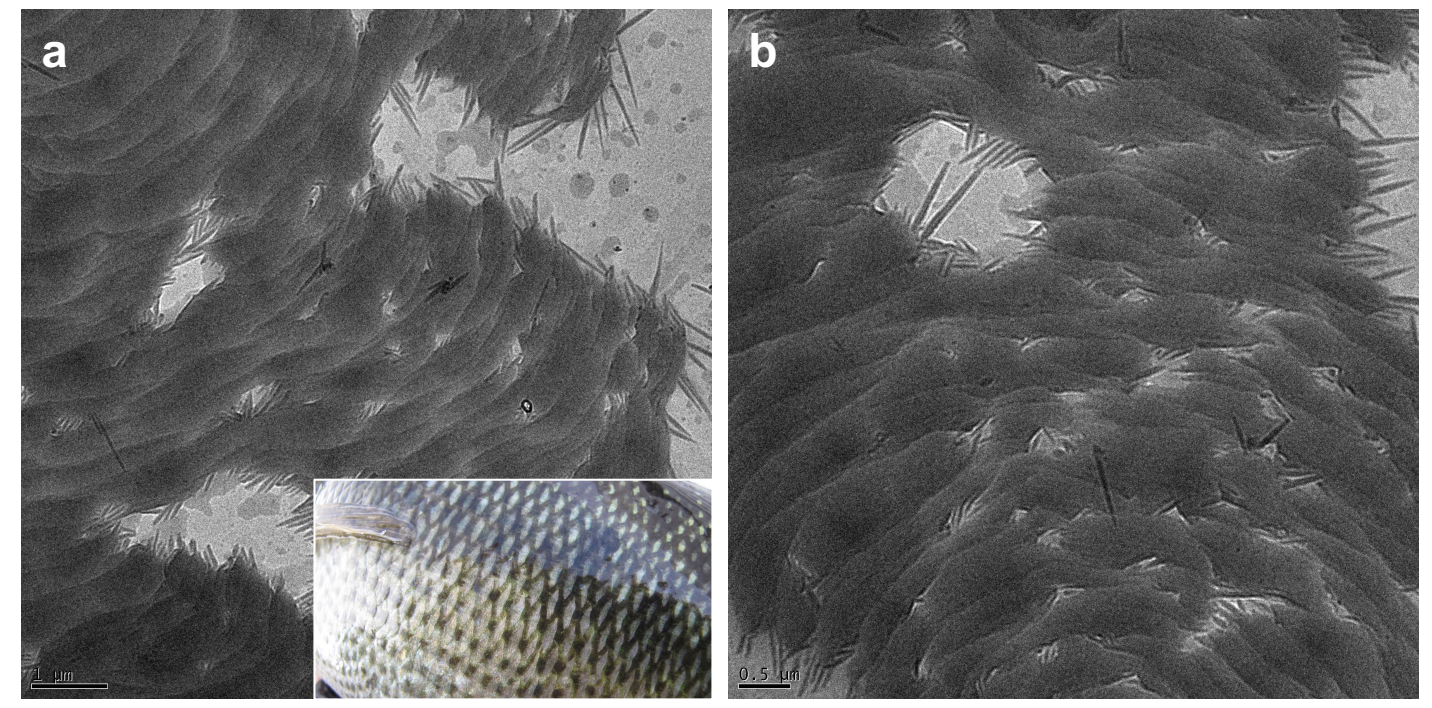

Figure S38. TEM images of $\mathbf{5}$ taken after rapid cooling from the isotropic liquid phase to room temperature: (a) scale bar $=1 \mu \mathrm{m}$ and (b) scale bar $=500 \mathrm{~nm}$; inset in (a) shows the scales of a fish. 


\section{Additional XRD data}

a

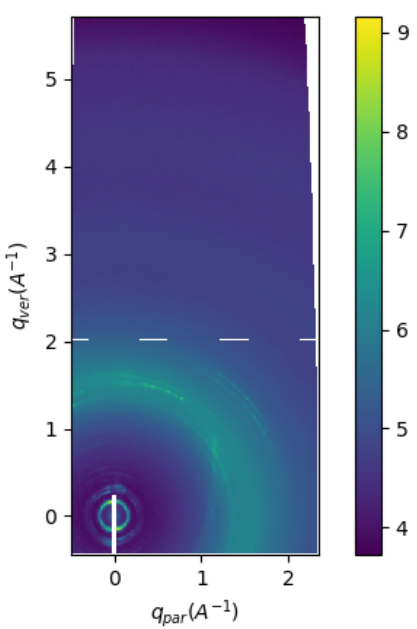

c

\begin{tabular}{|c|c|c|c|}
\hline$q / \AA^{-1}$ & & rel. intensity & index \\
\hline 0.163 & $q_{1}^{a}$ & $4.43 \mathrm{E}+09$ & 101 \\
\hline 0.170 & $q_{2}^{a}$ & $2.04 \mathrm{E}+09$ & 002 \\
\hline 0.276 & $q_{3}^{a}$ & $4.032 \mathrm{E}+08$ & 200 \\
\hline 0.326 & $q_{4}^{a}$ & $2.15 \mathrm{E}+08$ & 202 \\
\hline 0.341 & $q_{5}^{a}$ & $4.18 \mathrm{E}+08$ & 004 \\
\hline 0.370 & $q_{6}^{a}$ & $1.43 \mathrm{E}+08$ & 203 \\
\hline 0.426 & $q_{7}^{a}$ & $1.65 \mathrm{E}+08$ & 301 \\
\hline 0.454 & $q_{8}^{a}$ & $1.59 \mathrm{E}+08$ & 105 \\
\hline 0.477 & $q_{9}^{a}$ & $1.77 \mathrm{E}+08$ & 303 \\
\hline
\end{tabular}

b

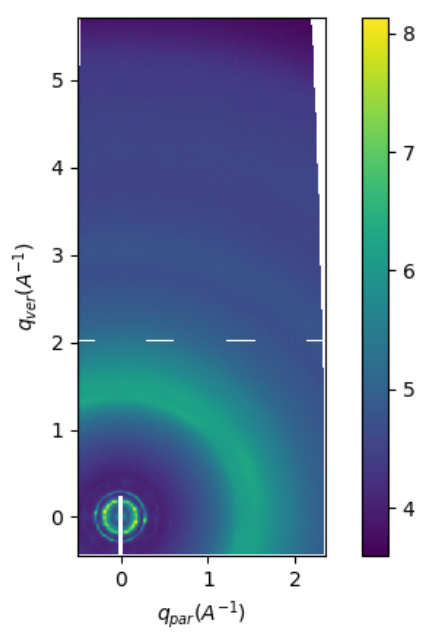

d

\begin{tabular}{|c|c|c|c|c|}
\hline \multirow{2}{*}{$q / \AA^{-1}$} & & \multirow{2}{*}{ rel. intensity } & \multicolumn{2}{|c|}{ index } \\
\hline & & & HLCN & Colr \\
\hline 0.143 & $q_{1}^{b}$ & $6.92 \mathrm{E}+08$ & 001 & \\
\hline 0.183 & $q_{1}^{c}$ & $1.09 \mathrm{E}+09$ & & 001 \\
\hline 0.185 & $q_{2}^{c}$ & $9.98 \mathrm{E}+08$ & & 100 \\
\hline 0.283 & $q_{3}^{c}$ & $7.78 \mathrm{E}+09$ & & 101 \\
\hline 0.286 & $q_{2}^{b}$ & $2.80 \mathrm{E}+00$ & 002 & \\
\hline 0.370 & $q_{4}^{c}$ & $1.61 \mathrm{E}+08$ & & 200 \\
\hline 0.429 & $q_{3}^{b}$ & $1.72 \mathrm{E}+08$ & 003 & \\
\hline 0.566 & $q_{5}^{c}$ & $1.67 E+08$ & & 202 \\
\hline 0.574 & $q_{6}^{c}$ & $1.72 \mathrm{E}+08$ & & 010 \\
\hline 0.64 & $q_{7}^{c}$ & $1.84 E+08$ & & 111 \\
\hline 0.865 & $q_{8}^{c}$ & $2.67 \mathrm{E}+08$ & & 303 \\
\hline
\end{tabular}

e

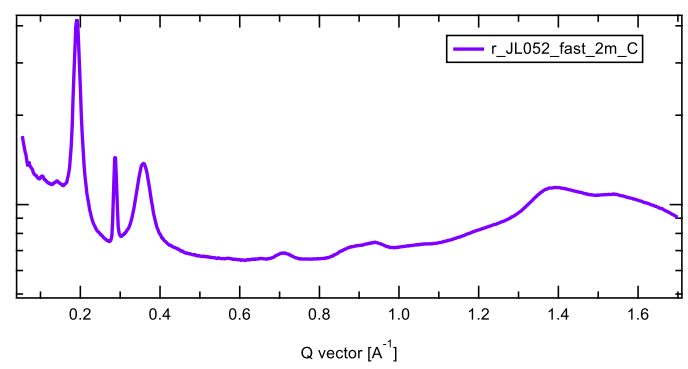

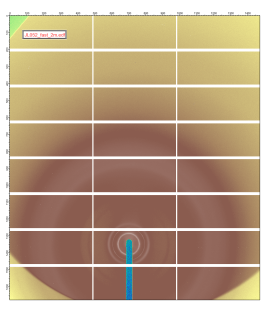

f

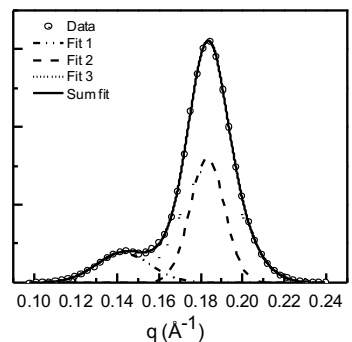

g

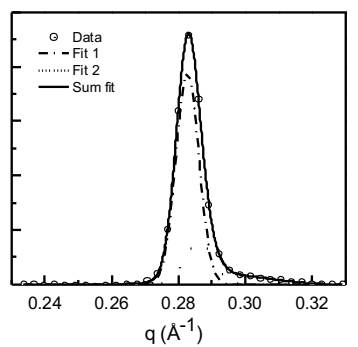

Figure S39. 2D XRD pattern obtained for compound 5 on: (a) slow cooling and (b) fast cooling. Momentum transfer $(q)$, Miller indices, and relative intensities on: (c) slow cooling and (d) fast cooling; all from BNL beamline. (e) Complementary ALS beamline data for compound 5 taken upon fast cooling from the isotropic liquid phase (used to further support the $\mathrm{B} 1 \mathrm{Col}_{\mathrm{ob}}-p 2$ phase assignment and indexing). Deconvolution of the scattering maxima: (f) $q_{1}^{\mathrm{c}}+q_{2}^{\mathrm{c}}$ and (g) $q_{3}^{\mathrm{c}}+q_{2}^{\mathrm{b}}$ (BNL dataset) recorded upon fast cooling (dataset shown in (b) and (d)). 
Section S7. Additional Data for Compounds $3-5$ in $n$-hexane dispersions Additional solution CD data
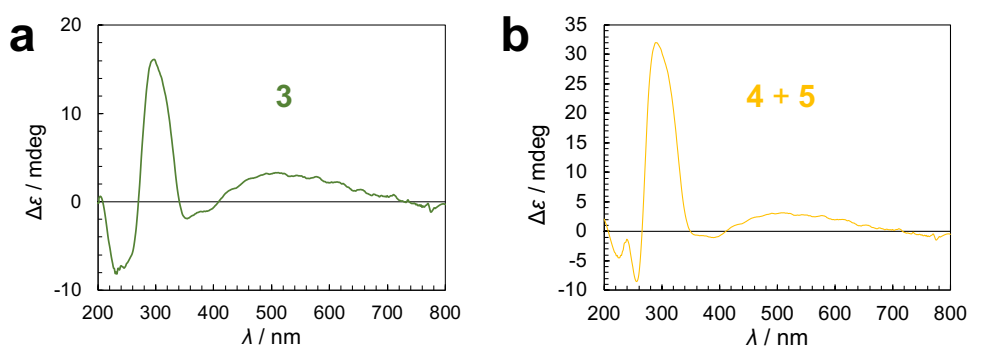

Figure S40. Clear additive nature of the solution CD data in $n$-hexane of: (a) compound 3 and (b) the sum of intensity-corrected spectra for compounds $\mathbf{4}$ and $\mathbf{5}$.

\section{SI References}

(1) Li, L.; Salamończyk, M.; Jákli, A.; Hegmann, T. A Dual Modulated Homochiral Helical Nanofilament Phase with Local Columnar Ordering Formed by Bent Core Liquid Crystals: Effects of Molecular Chirality. Small 2016, 12 (29), 3944-3955.

(2) Li, L.; Salamończyk, M.; Shadpour, S.; Zhu, C.; Jákli, A.; Hegmann, T. An Unusual Type of Polymorphism in a Liquid Crystal. Nat. Commun. 2018, 9 (1), 714.

(3) Shadpour, S.; Nemati, A.; Boyd, N. J.; Li, L.; Prévôt, M. E.; Wakerlin, S. L.; Vanegas, J. P.; Salamończyk, M.; Hegmann, E.; Zhu, C.; Wilson, M. R.; Jákli, A. I.; Hegmann, T., HeliconicalLayered Nanocylinders (HLNCs) - Hierarchical Self-Assembly in a Unique B4 Phase Liquid Crystal Morphology. Mater. Horiz. 2019, 6 (5), 959-968.

(4) Shadpour, S.; Nemati, A.; Salamończyk, M.; Prévôt, M. E.; Liu, J.; Boyd, N. J.; Wilson, M. R.; Zhu, C.; Hegmann, E.; Jákli, A. I.; Hegmann, T., Missing Link between Helical Nano- and Microfilaments in B4 Phase Bent-Core Liquid Crystals, and Deciphering Which Chiral Center Controls the Filament Handedness. Small 2020, 16 (4), 1905591.

(5) Zhang, C.; Diorio, N.; Lavrentovich, O. D.; Jákli, A. Helical Nanofilaments of Bent-Core Liquid Crystals with a Second Twist. Nat. Commun. 2014, 5, 3302. 